\title{
REB INDEXES
}

DOI: $10.20542 / 0202-179 X-2018-3 / 4-24-59$

Sergey Aukutsionek, Alexandra Batyaeva, Natalia Dyomina, Andrei Egorov, Alexander Matveev

Abstract: "REB indexes" cover a wide range of economic indicators of Russian industrial enterprises. The article presents basic statistical data collected on a monthly, quarterly and semi-annual base by the Russian Economic Barometer by means of direct questioning of managers of industrial and agricultural enterprises. Regular extension of rows allows seeing dynamics of more than 70 series of indicators and conducting comparative analysis of data collected since 1991.

Keywords: Russia; industry; industrial statistics; industrial enterprises; level of prices; wages; employment; production; investments; indebtedness to banks; orderbook level; stocks of finished products; capacity utilization.

Statistics of industrial enterprises of Russia

Diffusion indexes - actual, percent rising over 1-month spans:

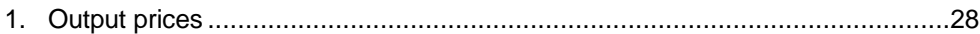

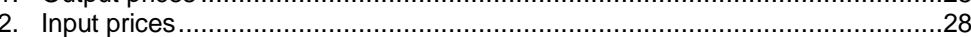

4. Wages ...

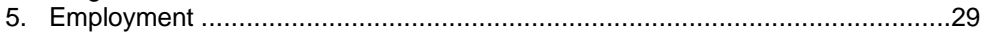

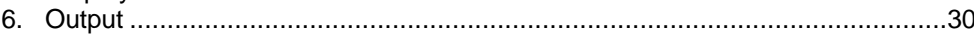

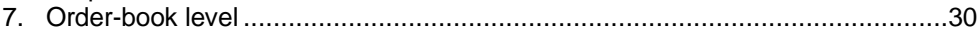

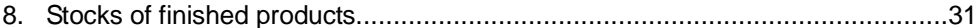

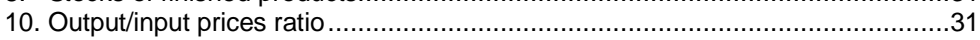

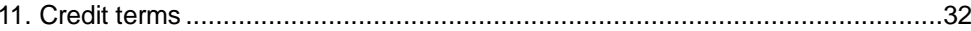

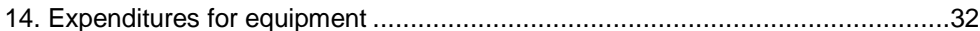

Diffusion indexes - anticipated, percent rising over 3-month spans:

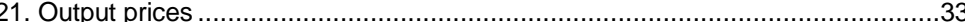

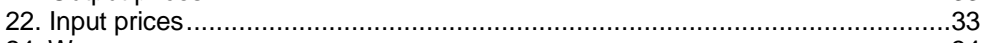

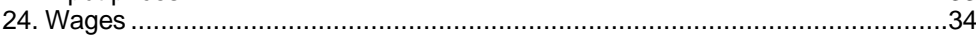

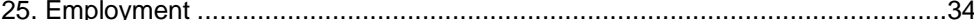

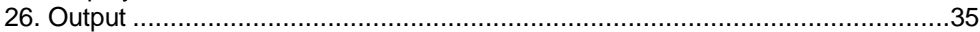

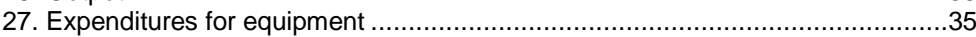

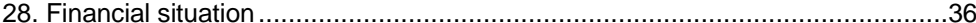

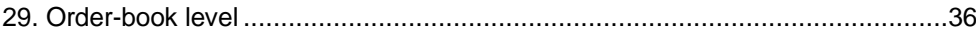

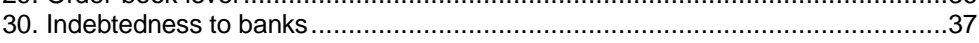

Normal monthly level = 100:

13. Capacity utilization rate 
15. Labour utilization rate...

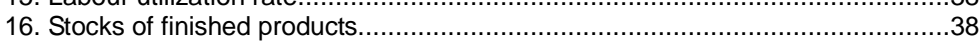

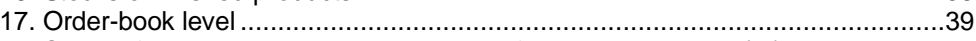

19. Share of enterprises in 'good' or 'normal' financial conditions (\%) .....................39

20. Share of enterprises not buying equipment for 2 and more months (\%) ..............40

31. Anticipated interest rates on bank credits (in roubles) to be received in the course of 3 months (\% on annual basis)

32. Share of enterprises not indebted to banks and not going to be indebted in the course of 3 months (\%)

3. Indebtedness to banks, industry (normal monthly level $=100$ )

34. Share of enterprises not going to make new bank borrowings in the next 3 months (\%)......

36. Share of sales for cash in total sales $(\%)$.

\section{Economic policy:}

41. Share of enterprise directors comprehending the government economic policy of the last 3-4 months (\%)

42. Share of enterprise directors not comprehending the government economic policy of the last 3-4 months (\%)

43. Index of comprehension of government's economic policy of the last 3-4 months

44. Share of enterprise directors approving of the government's economic policy of the last 3-4 months (\%)

45. Share of enterprise directors not approving of the government's economic policy of the last 3-4 months (\%)

. Index of approval of government's economic policy of the last 3-4 months 44

47. Time remaining until the end of crisis, managers' estimates (years) ..................44

Limitations to production - present situation, share of enterprises, \%:

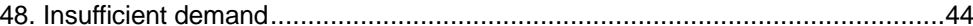

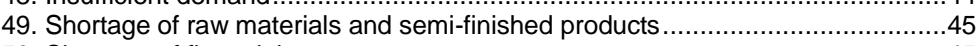

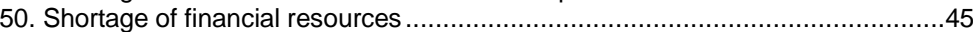

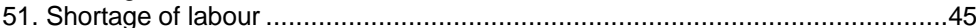

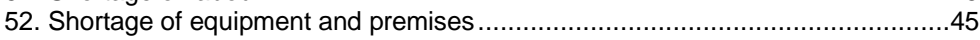

Limitations to capital investments - present situation, share of enterprises, \%:

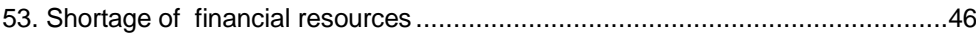

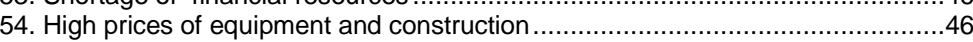

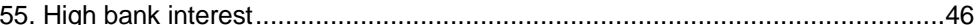

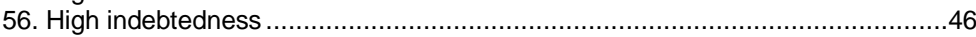

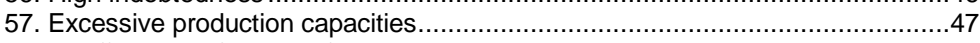

58. Insufficient profitability of investment projects ......................................... 47

The use of production capacities and workforce:

61. Percentage of enterprises with production capacities redundant against demand anticipated in 12 months .......................................................

Percentage of enterprises with prod
demand anticipated in 12 months

3. Percentage of enterprises with manpower redundant against demand anticipated in 12 months. 
64. Percentage of enterprises with manpower insufficient against demand anticipated in 12 months.................................................................. 48

65. Percentage of loss-making enterprises in the previous half-year, $\%$.................48

66. Percentage of enterprises likely to go bankrupt in the coming 1 to 2 years .......48

Job creation and destruction rates - 6 month spans, \%:

67. Job creation rate, actual

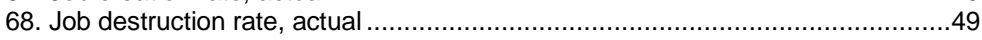

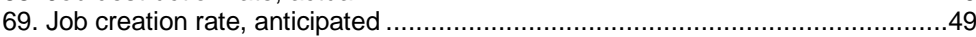

70. Job destruction rate, anticipated......................................................... 49

\section{Loss-making production, \%:}

71. Portion of loss-making production in the total output ................................. 50

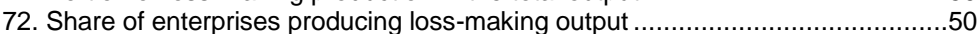

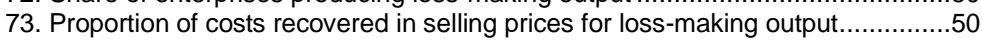

74. Loss ratio: portion of losses from loss-making output in total costs ...................50

Reasons for manufacturing loss-making production, \%:

175. Efforts to keep enterprises' market share

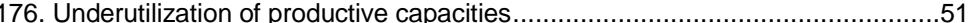

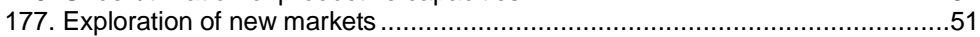

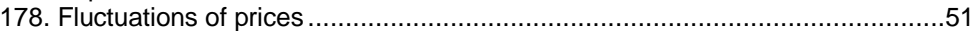

179. New and perhaps temporarily loss-making production ................................52

Investments, \%:

121. Share of enterprises with increased production capacities in the last 12

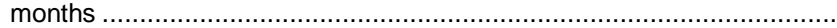

122. Share of enterprises with unchanged production capacities in the last 12

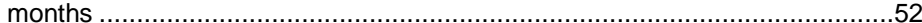

123. Share of enterprises with decreased production capacities in the last 12

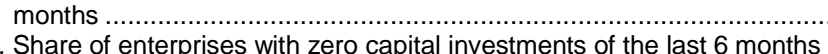

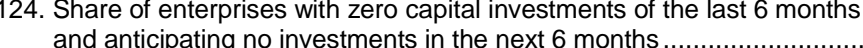

125. Investment plans fulfillment rate of the last 6 months (planned volume of investments $=100 \%$ )

126. Share of enterprises with no investment plans in the last 6 months

127. Marginal (affordable) interest rate for fixed investment (on a rouble loan for 2-3 years loan period)

Competition (a five-grade scale: 1 - absent, 5-menace of ousting from the market):

128. Competition on the main product markets ......

129. Competition with foreign makers on the main product markets .

Statistics of agricultural enterprises of Russia

Limitations to production - present situation, share of enterprises, \%:

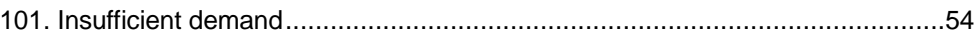

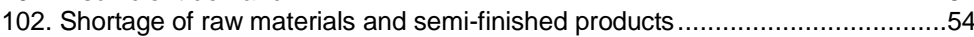

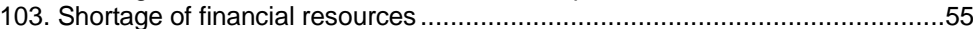


104. Shortage of labour ................................55

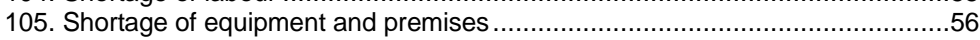

106. High prices for raw materials, semi-finished goods ................................56

\section{Economic policy:}

160. Share of enterprise directors comprehending the government economic policy of the last 3-4 months

161. Share of enterprise directors not comprehending the government economic policy of the last 3-4 months...

62. Index of comprehension of government's economic policy of the last 3-4

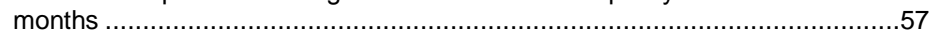

163. Share of enterprise directors approving of the government's economic

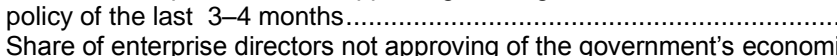

164. Share of enterprise directors not approving of the government's economic
policy of the last $3-4$ months

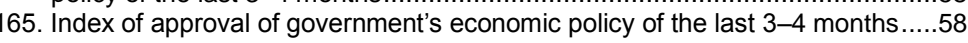

Limitations to capital investments - present situation, share of enterprises, \%:

166. Shortage of financial resources

167. High prices of equipment and construction

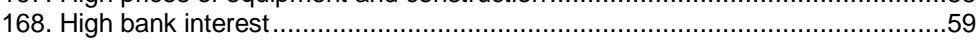

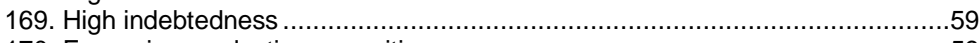

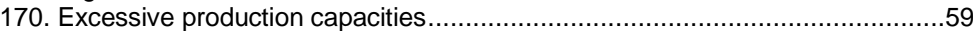

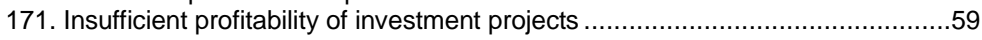




\begin{tabular}{|c|c|c|c|c|c|c|c|c|c|c|c|c|c|}
\hline Year & Jan. & Feb. & Mar. & Apr. & May & June & July & Aug. & Sept & Oct. & Nov. & Dec. & Annual \\
\hline \multicolumn{14}{|c|}{$\begin{array}{l}\text { 1. Diffusion index of output prices, industry, actual (percent rising over 1-month spans) } \\
\text { 1. Дифффузный индекс цен на выпускаемую продукцию, промышленность, фактические изменения } \\
\text { (доля предприятий с растущим за } 1 \text { месяц показателем) }\end{array}$} \\
\hline 1991 & - & - & - & - & - & - & - & - & - & - & - & 88 & - \\
\hline 1992 & 97 & 70 & 72 & 67 & 68 & 64 & 66 & 57 & 67 & 75 & 80 & 73 & 71 \\
\hline 1993 & 84 & 83 & 83 & 83 & 78 & 78 & 82 & 83 & 85 & 80 & 76 & 74 & 81 \\
\hline 1994 & 79 & 75 & 68 & 77 & 74 & 61 & 69 & 73 & 71 & 81 & 77 & 80 & 74 \\
\hline 1995 & 90 & 83 & 81 & 83 & 77 & 72 & 72 & 74 & 76 & 70 & 62 & 59 & 75 \\
\hline & 66 & 64 & 57 & 57 & 52 & 52 & 51 & 54 & 52 & 52 & 54 & 54 & 55 \\
\hline 1997 & 53 & 52 & 53 & 49 & 48 & 49 & 48 & 52 & 49 & 50 & 48 & 49 & 50 \\
\hline 1998 & 49 & 46 & 43 & 43 & 41 & 41 & 41 & 47 & 71 & 74 & 75 & 74 & 54 \\
\hline 1999 & 77 & 73 & 73 & 71 & 67 & 70 & 69 & 72 & 73 & 67 & 70 & 69 & 71 \\
\hline 2000 & 69 & 66 & 59 & 61 & 61 & 67 & 63 & 63 & 62 & 67 & 62 & 57 & 63 \\
\hline 2001 & 68 & 65 & 58 & 60 & 56 & 55 & 51 & 49 & 53 & 52 & 50 & 51 & 56 \\
\hline 2002 & 57 & 51 & 52 & 51 & 51 & 51 & 55 & 54 & 55 & 55 & 52 & 51 & 53 \\
\hline 2003 & 64 & 56 & 56 & 55 & 55 & 51 & 56 & 50 & 55 & 63 & 56 & 54 & 56 \\
\hline 2004 & 66 & 54 & 59 & 56 & 54 & 53 & 53 & 52 & 57 & 52 & 52 & 52 & 55 \\
\hline 2005 & 59 & 59 & 53 & 48 & 49 & 50 & 51 & 52 & 51 & 52 & 51 & 53 & 52 \\
\hline 2006 & 56 & 55 & 53 & 51 & 50 & 52 & 52 & 53 & 53 & 54 & 54 & 53 & 53 \\
\hline 2007 & 58 & 57 & 54 & 60 & 56 & 57 & 61 & 63 & 57 & 64 & 57 & 58 & 59 \\
\hline 2008 & 68 & 63 & 61 & 60 & 59 & 59 & 57 & 56 & 53 & 48 & 41 & 39 & 55 \\
\hline 2009 & 46 & 47 & 46 & 42 & 41 & 43 & 43 & 45 & 46 & 49 & 47 & 47 & 45 \\
\hline 2010 & 55 & 53 & 51 & 52 & 51 & 52 & 58 & 53 & 57 & 61 & 59 & 53 & 55 \\
\hline 2011 & 68 & 62 & 61 & 56 & 54 & 50 & 53 & 55 & 56 & 55 & 52 & 56 & 56 \\
\hline & 60 & 61 & 56 & 49 & 51 & 48 & 55 & 55 & 55 & 54 & 5 & 51 & 54 \\
\hline 201 & 53 & 52 & 47 & 47 & 46 & 52 & 51 & 53 & 51 & 52 & 52 & 51 & 51 \\
\hline 20 & 56 & 52 & 50 & 53 & 52 & 48 & 50 & 55 & 51 & 59 & 49 & 65 & 53 \\
\hline 20 & 78 & 63 & 63 & 55 & 49 & 48 & 54 & 54 & 52 & 51 & 52 & 52 & 56 \\
\hline 20 & 57 & 54 & 51 & 51 & 54 & 55 & 53 & 45 & 56 & 5 & 50 & 50 & 53 \\
\hline 2017 & 66 & 54 & 48 & 53 & 50 & 49 & 50 & 47 & 48 & 55 & 55 & 49 & 52 \\
\hline 2018 & 53 & 49 & 54 & 39 & 50 & 58 & 49 & 53 & & & & & \\
\hline \multirow{2}{*}{\multicolumn{14}{|c|}{$\begin{array}{l}\text { 2. Diffusion index of input prices, industry, actual (percent rising over 1-month spans) } \\
\text { 2. Диффузный индекс цен на покупаемую продукцию, промышленность, фактические изменения (доля } \\
\text { предприятий с растущим за } 1 \text { месяц показателем) }\end{array}$}} \\
\hline & & & & & & & & & & & & & \\
\hline 1991 & - & - & - & - & - & - & - & - & - & - & - & 99 & - \\
\hline 1992 & 97 & 94 & $\overline{91}$ & 87 & 89 & 90 & 85 & 82 & 85 & 89 & 93 & 89 & \\
\hline 1993 & 95 & 93 & 94 & 96 & 94 & 92 & 91 & 94 & 96 & 94 & 95 & 92 & 94 \\
\hline 4 & 95 & 95 & 94 & 97 & 96 & 92 & 94 & 93 & 93 & 97 & 94 & 95 & 95 \\
\hline & 98 & 97 & 96 & 97 & 95 & 93 & 9 & 93 & 94 & 9 & 8 & 87 & 93 \\
\hline 19 & 89 & 86 & 87 & 84 & 84 & 81 & 80 & 80 & 84 & 7 & 79 & 74 & 82 \\
\hline & 74 & 77 & 80 & 77 & 74 & 75 & 75 & 78 & 64 & 6 & 6 & 66 & 02 \\
\hline & 63 & 59 & 60 & 60 & 60 & 58 & 54 & 68 & 91 & 92 & 9 & 93 & 71 \\
\hline & 94 & 94 & 92 & 91 & 90 & 9 & $8 \mathrm{~s}$ & 94 & 92 & 89 & 8 & 86 & \\
\hline 2000 & 89 & 87 & 87 & 84 & 88 & 87 & 88 & 85 & 86 & 86 & 8 & 82 & 86 \\
\hline & 88 & 85 & 8 & 81 & $7 \subseteq$ & 7 & 6 & 6 & 75 & 68 & 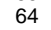 & 63 & \\
\hline & 71 & 67 & 6 & 6 & 6 & 68 & 7 & 68 & 70 & 7 & 6 & 7 & b \\
\hline & 80 & 79 & 7 & 73 & 74 & 6 & $6 \subseteq$ & 65 & 72 & 71 & 7 & 70 & 72 \\
\hline & 82 & 74 & $7 \varepsilon$ & 73 & 71 & 6 & 6 & 6 & 71 & 7 & 6 & 65 & 71 \\
\hline 20 & 77 & 60 & 67 & 65 & 62 & 6 & 6 & 6 & 65 & 67 & 6 & 64 & 65 \\
\hline & 76 & 70 & 70 & 5 & $5 \varepsilon$ & 6 & 6 & 6 & 6 & 65 & 0 & 6 & 66 \\
\hline & 74 & 70 & 68 & 7 & 72 & 6 & 73 & 7 & 75 & 78 & 7 & 76 & 73 \\
\hline & 84 & 80 & 80 & 82 & 80 & 7 & 7) & 7 & 73 & 65 & 5 & 49 & 73 \\
\hline & 75 & 76 & 70 & 61 & 61 & 6 & 6 & 71 & 74 & 6 & 6 & 71 & 69 \\
\hline 20 & 74 & 74 & 72 & 76 & 74 & 73 & $7 \varepsilon$ & 74 & 79 & 80 & 8 & 81 & 77 \\
\hline 20 & 90 & 83 & 7 & 74 & 71 & 71 & 70 & 72 & 73 & 70 & 6 & 65 & 74 \\
\hline 20 & 70 & 73 & 6 & 6 & 7 & 7 & 7 & 75 & 73 & 71 & 6 & 68 & 71 \\
\hline & 78 & 72 & 65 & 64 & 63 & 66 & 71 & 73 & 67 & 67 & 6 & 68 & 68 \\
\hline 20 & 81 & 72 & 76 & 73 & 73 & 70 & 70 & 77 & 82 & 82 & $8 \varepsilon$ & 90 & 78 \\
\hline 2015 & 94 & 83 & 79 & 70 & 74 & 72 & 75 & 86 & 80 & 81 & 78 & 71 & 79 \\
\hline 20 & 83 & 74 & 75 & 75 & 78 & 71 & 69 & 67 & 73 & 73 & 71 & 73 & 74 \\
\hline 2017 & 78 & 76 & 71 & 69 & 71 & 72 & 66 & 70 & 71 & 72 & 69 & 68 & 71 \\
\hline 2018 & 66 & 72 & 72 & 74 & 82 & 71 & 80 & 82 & & & & & \\
\hline
\end{tabular}




\begin{tabular}{|c|c|c|c|c|c|c|c|c|c|c|c|c|c|}
\hline Year & Jan. & Feb. & Mar. & Apr. & May & June & July & Aug. & Sept & Oct. & Nov. & Dec. & Annual \\
\hline \multicolumn{14}{|c|}{$\begin{array}{l}\text { 4. Diffusion index of wages, industry, actual (percent rising over 1-month spans) } \\
\text { 4. Диффуузый индекс заработной платы, промышленность, фактические изменения (доля } \\
\text { предприятий с растущим за } 1 \text { месяц показателем) }\end{array}$} \\
\hline 1991 & - & - & - & - & - & - & - & - & - & - & - & 92 & - \\
\hline 1992 & 94 & 78 & 77 & 65 & 66 & 81 & 69 & 60 & 67 & 72 & 73 & 77 & 73 \\
\hline 1993 & 75 & 79 & 80 & 84 & 82 & 81 & 82 & 76 & 77 & 74 & 67 & 67 & 77 \\
\hline 1994 & 62 & 52 & 56 & 60 & 55 & 60 & 63 & 58 & 61 & 68 & 67 & 69 & 61 \\
\hline 1995 & 60 & 64 & 67 & 68 & 64 & 70 & 69 & 66 & 65 & 62 & 55 & 54 & 64 \\
\hline 1996 & 47 & 57 & 50 & 50 & 42 & 49 & 48 & 45 & 46 & 48 & 37 & 43 & 47 \\
\hline 1997 & 41 & 43 & 51 & 50 & 46 & 57 & 54 & 51 & 53 & 49 & 39 & 45 & 48 \\
\hline 1998 & 40 & 47 & 43 & 43 & 35 & 42 & 37 & 40 & 44 & 49 & 47 & 61 & 44 \\
\hline 1999 & 52 & 61 & 64 & 67 & 59 & 68 & 65 & 66 & 68 & 63 & 65 & 67 & 64 \\
\hline 2000 & 53 & 61 & 62 & 59 & 59 & 65 & 64 & 67 & 65 & 63 & 58 & 62 & 62 \\
\hline 2001 & 56 & 59 & 60 & 64 & 61 & 60 & 58 & 58 & 61 & 58 & 51 & 54 & 58 \\
\hline 2002 & 44 & 51 & 54 & 54 & 45 & 51 & 58 & 54 & 52 & 55 & 49 & 54 & 52 \\
\hline 2003 & 41 & 49 & 56 & 57 & 53 & 56 & 51 & 54 & 50 & 56 & 47 & 56 & 52 \\
\hline 2004 & 47 & 55 & 59 & 57 & 51 & 56 & 56 & 50 & 48 & 50 & 42 & 54 & 52 \\
\hline 2005 & 40 & 50 & 58 & 54 & 53 & 54 & 55 & 58 & 53 & 48 & 48 & 56 & 52 \\
\hline 2006 & 44 & 51 & 59 & 52 & 58 & 59 & 62 & 62 & 55 & 54 & 53 & 60 & 56 \\
\hline 2007 & 44 & 53 & 61 & 61 & 61 & 57 & 65 & 66 & 61 & 64 & 58 & 60 & 59 \\
\hline 2008 & 50 & 61 & 63 & 64 & 58 & 60 & 61 & 62 & 60 & 51 & 34 & 36 & 55 \\
\hline 2009 & 24 & 28 & 41 & 42 & 40 & 45 & 44 & 48 & 46 & 45 & 44 & 49 & 41 \\
\hline 2010 & 33 & 52 & 54 & 54 & 51 & 55 & 59 & 53 & 53 & 52 & 51 & 57 & 52 \\
\hline 2011 & 43 & 58 & 59 & 55 & 61 & 56 & 53 & 55 & 55 & 56 & 54 & 55 & 55 \\
\hline 2012 & 42 & 52 & 60 & 59 & 65 & 57 & 66 & 63 & 54 & 53 & 47 & 49 & 56 \\
\hline 2013 & 43 & 51 & 60 & 53 & 49 & 57 & 64 & 54 & 51 & 55 & 49 & 58 & 54 \\
\hline 2014 & 46 & 47 & 49 & 52 & 58 & 57 & 55 & 47 & 47 & 51 & 48 & 54 & 51 \\
\hline 2015 & 40 & 49 & 57 & 46 & 33 & 57 & 50 & 54 & 40 & 48 & 50 & 49 & 48 \\
\hline 2016 & 35 & 50 & 48 & 52 & 43 & 55 & 52 & 50 & 54 & 53 & 57 & 61 & 51 \\
\hline 2017 & 40 & 52 & 57 & 50 & 50 & 53 & 55 & 52 & 45 & 53 & 45 & 54 & 51 \\
\hline 2018 & 44 & 59 & 43 & 43 & 60 & 64 & 53 & 47 & & & & & \\
\hline \multicolumn{14}{|c|}{$\begin{array}{l}\text { 5. Diffusion index of employment, industry, actual (percent rising over 1-month spans) } \\
\text { 5. Дифрфузный индекс занятости, промышленность, фактические изменения } \\
\text { (доля предприятий с растущим за } 1 \text { месяц показателем) }\end{array}$} \\
\hline 1991 & - & - & - & - & - & - & - & - & - & - & - & 55 & $\overline{-}$ \\
\hline 1992 & 53 & 41 & 37 & 36 & 32 & 30 & 28 & 27 & 26 & 30 & $\overline{31}$ & 30 & $\overline{33}$ \\
\hline 1993 & 33 & 32 & 35 & 33 & 35 & 32 & 40 & 33 & 37 & 32 & 30 & 29 & 33 \\
\hline 1994 & 30 & 26 & 26 & 27 & 22 & 24 & 22 & 21 & 24 & 22 & 25 & 33 & 25 \\
\hline 1995 & 34 & 33 & 34 & 33 & 32 & 35 & 39 & 33 & 37 & 32 & 33 & 29 & 34 \\
\hline 1996 & 27 & 29 & 26 & 31 & 29 & 30 & 29 & 24 & 25 & 27 & 30 & 28 & 28 \\
\hline 1997 & 30 & 31 & 30 & 33 & 31 & 38 & 32 & 35 & 35 & 31 & 37 & 38 & 33 \\
\hline 1998 & 35 & 37 & 33 & 33 & 30 & 33 & 32 & 31 & 29 & 35 & 36 & 36 & 33 \\
\hline 1999 & 38 & 41 & 42 & 43 & 45 & 46 & 46 & 44 & 44 & 45 & 47 & 43 & 44 \\
\hline 2000 & 43 & 46 & 43 & 44 & 44 & 45 & 46 & 41 & 45 & 43 & 44 & 45 & 44 \\
\hline 2001 & 43 & 43 & 42 & 43 & 41 & 41 & 40 & 40 & 36 & 40 & 42 & 38 & 41 \\
\hline 2002 & 40 & 39 & 42 & 39 & 33 & 36 & 38 & 37 & 34 & 37 & 36 & 35 & 37 \\
\hline 2003 & 32 & 33 & 38 & 34 & 35 & 39 & 36 & 39 & 38 & 39 & 38 & 37 & 37 \\
\hline 2004 & 40 & 41 & 42 & 40 & 43 & 45 & 45 & 48 & 43 & 46 & 41 & 45 & 43 \\
\hline 2005 & 39 & 40 & 42 & 40 & 39 & 39 & 43 & 42 & 41 & 43 & 41 & 42 & 41 \\
\hline 2006 & 40 & 49 & 45 & 48 & 45 & 45 & 45 & 49 & 48 & 47 & 49 & 52 & 47 \\
\hline 2007 & 46 & 49 & 49 & 48 & 47 & 48 & 50 & 49 & 45 & 49 & 52 & 50 & 49 \\
\hline 2008 & 48 & 52 & 46 & 43 & 44 & 39 & 42 & 42 & 37 & 38 & 32 & 31 & 41 \\
\hline 2009 & 29 & 28 & 3 & 37 & 31 & 31 & 32 & 35 & 40 & 38 & 45 & 39 & 35 \\
\hline 2010 & 37 & 43 & 43 & 45 & 41 & 48 & 47 & 45 & 46 & 49 & 47 & 47 & 45 \\
\hline 2011 & 46 & 48 & 51 & 49 & 48 & 45 & 52 & 48 & 44 & 50 & 51 & 45 & 48 \\
\hline 2012 & 44 & 45 & 50 & 49 & 42 & 42 & 45 & 50 & 44 & 45 & 46 & 44 & 46 \\
\hline 2013 & 45 & 47 & 5 & 46 & 44 & 42 & 37 & 41 & 38 & 44 & 46 & 40 & 43 \\
\hline 2014 & 40 & 44 & 49 & 45 & 39 & 43 & 45 & 41 & 40 & 38 & 37 & 36 & 41 \\
\hline 2015 & 43 & 45 & 50 & 44 & 50 & 40 & 45 & 44 & 41 & 46 & 41 & 43 & 44 \\
\hline 2016 & 39 & 49 & 41 & 39 & 39 & 51 & 47 & 44 & 42 & 50 & 48 & 51 & 45 \\
\hline 2017 & 42 & 48 & 45 & 50 & 40 & 45 & 44 & 52 & 37 & 42 & 45 & 46 & 45 \\
\hline 2018 & 45 & 53 & 47 & 44 & 46 & 50 & 44 & 42 & & & & & \\
\hline
\end{tabular}




\begin{tabular}{|c|c|c|c|c|c|c|c|c|c|c|c|c|c|}
\hline Year & Jan. & Feb. & Mar. & Apr. & May & June & July & Aug. & Sept & Oct. & Nov. & Dec. & Annual \\
\hline \multicolumn{14}{|c|}{$\begin{array}{l}\text { 6. Diffusion index of output, industry, actual (percent rising over 1-month spans) } \\
\text { 6. Диффузный индекс выпуска, промышленность, фактические изменения } \\
\text { (доля предприятий с растущим за } 1 \text { месяц показателем) }\end{array}$} \\
\hline 1992 & 44 & 42 & 39 & 48 & 36 & 36 & 37 & 31 & 31 & 48 & 35 & 54 & 40 \\
\hline 1993 & 35 & 51 & 55 & 49 & 46 & 43 & 43 & 40 & 47 & 40 & 32 & 37 & 43 \\
\hline 1994 & 31 & 35 & 32 & 32 & 26 & 38 & 29 & 39 & 34 & 41 & 41 & 40 & 35 \\
\hline 1995 & 31 & 46 & 47 & 33 & 38 & 46 & 42 & 45 & 35 & 39 & 28 & 31 & 38 \\
\hline 1996 & 29 & 41 & 32 & 36 & 30 & 32 & 31 & 30 & 30 & 37 & 26 & 36 & 32 \\
\hline 1997 & 20 & 34 & 44 & 39 & 35 & 43 & 41 & 36 & 47 & 41 & 36 & 51 & 39 \\
\hline 1998 & 28 & 43 & 43 & 38 & 30 & 42 & 32 & 32 & 32 & 42 & 38 & 51 & 38 \\
\hline 1999 & 33 & 55 & 54 & 47 & 39 & 53 & 48 & 51 & 56 & 48 & 49 & 57 & 49 \\
\hline 2000 & 34 & 54 & 56 & 44 & 47 & 50 & 50 & 52 & 41 & 48 & 45 & 45 & 47 \\
\hline 2001 & 35 & 45 & 51 & 48 & 40 & 44 & 44 & 54 & 39 & 48 & 38 & 37 & 44 \\
\hline 2002 & 31 & 42 & 48 & 45 & 37 & 42 & 45 & 46 & 43 & 51 & 39 & 49 & 43 \\
\hline 2003 & 29 & 47 & 55 & 50 & 36 & 54 & 48 & 39 & 46 & 49 & 37 & 51 & 45 \\
\hline 2004 & 35 & 43 & 57 & 46 & 37 & 53 & 44 & 43 & 38 & 37 & 34 & 47 & 43 \\
\hline 2005 & 18 & 49 & 60 & 47 & 43 & 52 & 44 & 52 & 47 & 40 & 44 & 44 & 45 \\
\hline 2006 & 21 & 58 & 65 & 48 & 54 & 52 & 49 & 61 & 49 & 53 & 48 & 53 & 51 \\
\hline 2007 & 26 & 59 & 62 & 55 & 55 & 53 & 54 & 62 & 42 & 55 & 49 & 49 & 52 \\
\hline 2008 & 29 & 62 & 62 & 56 & 47 & 45 & 53 & 49 & 44 & 44 & 19 & 26 & 45 \\
\hline 2009 & 16 & 35 & 41 & 38 & 33 & 39 & 46 & 39 & 41 & 49 & 38 & 47 & 39 \\
\hline 2010 & 21 & 56 & 63 & 51 & 40 & 55 & 46 & 40 & 49 & 50 & 45 & 49 & 47 \\
\hline 2011 & 28 & 57 & 64 & 48 & 52 & 55 & 38 & 59 & 47 & 55 & 50 & 48 & 50 \\
\hline 2012 & 25 & 60 & 59 & 57 & 59 & 44 & 55 & 52 & 43 & 58 & 46 & 41 & 50 \\
\hline 2013 & 32 & 51 & 54 & 43 & 41 & 53 & 53 & 43 & 35 & 49 & 41 & 47 & 45 \\
\hline 2014 & 23 & 54 & 44 & 53 & 38 & 56 & 40 & 41 & 37 & 40 & 40 & 48 & 43 \\
\hline 2015 & 28 & 51 & 49 & 32 & 34 & 45 & 39 & 42 & 35 & 46 & 36 & 52 & 41 \\
\hline 2016 & 22 & 51 & 43 & 46 & 36 & 50 & 44 & 37 & 51 & 49 & 45 & 55 & 44 \\
\hline 2017 & 26 & 42 & 61 & 49 & 41 & 45 & 47 & $39^{*}$ & 41 & 51 & 41 & 43 & 44 \\
\hline 2018 & 34 & 55 & 40 & 33 & 38 & 57 & 40 & 39 & & & & & \\
\hline \multirow{2}{*}{\multicolumn{14}{|c|}{$\begin{array}{l}\text { 7. Diffusion index of order-book level, industry, actual (percent rising over 1-month spans) } \\
\text { 7. Диффузный индекс портфеля заказов, промышленность, фактические изменения } \\
\text { (доля предприятий с растущим за } 1 \text { месяц показателем) }\end{array}$}} \\
\hline & & & & & & & & & & & & & \\
\hline 1991 & - & - & - & - & - & - & - & - & - & - & - & 61 & - \\
\hline 1992 & 32 & 29 & 29 & 30 & 25 & 32 & 27 & 31 & 31 & 42 & 40 & 48 & 33 \\
\hline 1993 & 41 & 49 & 46 & 42 & 45 & 44 & 36 & 37 & 46 & 35 & 34 & 34 & 41 \\
\hline 1994 & 33 & 33 & 31 & 31 & 31 & 40 & 36 & 41 & 43 & 39 & 41 & 47 & 37 \\
\hline 1995 & 44 & 44 & 42 & 42 & 43 & 48 & 42 & 45 & 38 & 34 & 31 & 31 & 40 \\
\hline 1996 & 32 & 36 & 31 & 33 & 36 & 37 & 32 & 34 & 35 & 35 & 31 & 37 & 34 \\
\hline 1997 & 34 & 40 & 47 & 46 & 44 & 41 & 44 & 45 & 45 & 42 & 44 & 42 & 43 \\
\hline 1998 & 42 & 42 & 41 & 41 & 37 & 38 & 36 & 38 & 40 & 45 & 46 & 52 & 42 \\
\hline 1999 & 50 & 58 & 60 & 56 & 54 & 60 & 58 & 62 & 61 & 52 & 51 & 49 & 56 \\
\hline 2000 & 47 & 54 & 57 & 53 & 53 & 55 & 59 & 57 & 52 & 48 & 43 & 45 & 52 \\
\hline 2001 & 44 & 49 & 55 & 50 & 45 & 50 & 52 & 56 & 46 & 52 & 42 & 40 & 48 \\
\hline 2002 & 41 & 44 & 49 & 48 & 47 & 49 & 54 & 52 & 47 & 45 & 39 & 45 & 47 \\
\hline 2003 & 35 & 49 & 50 & 51 & 45 & 56 & 55 & 51 & 53 & 52 & 44 & 47 & 49 \\
\hline 2004 & 40 & 48 & 55 & 52 & 46 & 59 & 51 & 51 & 41 & 41 & 37 & 49 & 48 \\
\hline 2005 & 28 & 50 & 59 & 51 & 51 & 52 & 50 & 48 & 50 & 44 & 45 & 53 & 48 \\
\hline 2006 & 31 & 56 & 62 & 47 & 60 & 5 & 55 & 60 & 56 & 54 & 5 & 51 & 53 \\
\hline 20 & 38 & 58 & 62 & 57 & 54 & 5 & 57 & 58 & 49 & 52 & 46 & 51 & 53 \\
\hline 2008 & 38 & 56 & 57 & 50 & 48 & 52 & 52 & 49 & 42 & 30 & 18 & 18 & 43 \\
\hline 2009 & 19 & 27 & 34 & 33 & 32 & 40 & 39 & 41 & 49 & 38 & 39 & 43 & 36 \\
\hline 201 & 32 & 49 & 50 & 56 & 54 & 5 & 51 & 50 & 55 & 48 & 38 & 45 & 48 \\
\hline & 40 & 54 & 56 & 47 & 57 & 49 & 49 & 5 & 50 & 48 & 4 & 48 & 50 \\
\hline & 32 & 51 & 56 & 56 & 61 & 43 & 53 & 55 & 47 & 53 & 45 & 39 & 49 \\
\hline & 37 & 49 & 47 & 45 & 41 & 43 & 46 & 38 & 45 & 44 & 39 & 40 & 43 \\
\hline & 36 & 43 & 43 & 49 & 36 & 48 & 41 & 44 & 40 & 42 & 36 & 43 & 42 \\
\hline & 28 & 49 & 46 & 48 & 41 & 46 & 42 & 48 & 40 & 44 & 40 & 47 & 43 \\
\hline 20 & 34 & 46 & 48 & 48 & 54 & 51 & 47 & 44 & 51 & 41 & 47 & 45 & 46 \\
\hline 201 & 33 & 44 & 47 & 45 & 46 & 47 & 44 & 42 & 44 & 41 & 44 & 37 & 43 \\
\hline 2018 & 40 & 47 & 42 & 44 & 41 & 50 & 40 & 34 & & & & & \\
\hline
\end{tabular}

Corrected. Here and afterwards the asterisk after the numeric indicates that it has been recalculated and changed. 


\begin{tabular}{|c|c|c|c|c|c|c|c|c|c|c|c|c|c|}
\hline Year & Jan. & Feb. & Mar. & Apr. & May & June & July & Aug. & Sept & Oct. & Nov. & Dec. & Annual \\
\hline \multicolumn{14}{|c|}{$\begin{array}{l}\text { 8. Diffusion index of stocks of finished products, industry, actual (percent rising over 1-month spans) } \\
\text { 8. Дифффузный индекс запасов готовой продукции, промышленность, фактические изменения (доля } \\
\text { предприятий с растущим за } 1 \text { месяц показателем) }\end{array}$} \\
\hline 1992 & 56 & 76 & 75 & 72 & 70 & 67 & 73 & 66 & 62 & 58 & 53 & 51 & 65 \\
\hline 1993 & 56 & 55 & 61 & 57 & 57 & 58 & 48 & 64 & 57 & 69 & 61 & 67 & 59 \\
\hline 1994 & 59 & 61 & 61 & 65 & 55 & 50 & 44 & 47 & 43 & 41 & 39 & 38 & 50 \\
\hline 1995 & 48 & 46 & 55 & 49 & 50 & 44 & 43 & 46 & 50 & 56 & 52 & 60 & 50 \\
\hline 1996 & 58 & 57 & 56 & 56 & 50 & 52 & 46 & 44 & 43 & 49 & 43 & 47 & 50 \\
\hline 1997 & 44 & 40 & 51 & 46 & 42 & 41 & 36 & 38 & 37 & 45 & 43 & 43 & 42 \\
\hline 1998 & 44 & 51 & 52 & 46 & 46 & 43 & 43 & 37 & 31 & 36 & 36 & 35 & 42 \\
\hline 1999 & 40 & 40 & 37 & 38 & 38 & 37 & 36 & 32 & 35 & 39 & 37 & 39 & 37 \\
\hline 2000 & 41 & 41 & 40 & 45 & 46 & 39 & 41 & 36 & 38 & 44 & 48 & 45 & 42 \\
\hline 2001 & 51 & 51 & 53 & 50 & 51 & 46 & 38 & 42 & 46 & 45 & 48 & 51 & 48 \\
\hline 2002 & 55 & 55 & 52 & 46 & 47 & 44 & 41 & 40 & 42 & 46 & 48 & 40 & 46 \\
\hline 2003 & 46 & 54 & 50 & 51 & 42 & 48 & 39 & 42 & 39 & 42 & 53 & 46 & 46 \\
\hline 2004 & 53 & 48 & 44 & 49 & 42 & 44 & 51 & 40 & 44 & 44 & 52 & 49 & 47 \\
\hline 2005 & 61 & 51 & 46 & 48 & 50 & 43 & 42 & 39 & 41 & 46 & 44 & 45 & 46 \\
\hline 2006 & 55 & 52 & 52 & 51 & 45 & 45 & 45 & 38 & 39 & 41 & 37 & 43 & 45 \\
\hline 2007 & 44 & 44 & 46 & 45 & 39 & 41 & 39 & 42 & 40 & 38 & 43 & 41 & 42 \\
\hline 2008 & 49 & 51 & 47 & 53 & 53 & 48 & 43 & 53 & 49 & 55 & 63 & 57 & 52 \\
\hline 2009 & 59 & 52 & 47 & 48 & 50 & 32 & 44 & 41 & 42 & 41 & 42 & 41 & 45 \\
\hline 2010 & 47 & 50 & 45 & 41 & 46 & 40 & 48 & 36 & 39 & 40 & 46 & 38 & 43 \\
\hline 2011 & 50 & 48 & 50 & 58 & 52 & 49 & 45 & 44 & 43 & 44 & 46 & 51 & 48 \\
\hline 2012 & 52 & 42 & 54 & 47 & 46 & 52 & 41 & 42 & 42 & 41 & 47 & 51 & 46 \\
\hline 2013 & 54 & 54 & 46 & 46 & 54 & 56 & 47 & 43 & 50 & 43 & 49 & 51 & 49 \\
\hline 2014 & 55 & 54 & 54 & 48 & 50 & 40 & 47 & 53 & 43 & 44 & 43 & 40 & 48 \\
\hline 2015 & 45 & 50 & 51 & 53 & 61 & 55 & 48 & 46 & 52 & 38 & 53 & 42 & 49 \\
\hline 2016 & 50 & 49 & 56 & 54 & 47 & 50 & 55 & 47 & 47 & 47 & 42 & 45 & 49 \\
\hline 2017 & 56 & 49 & 50 & 52 & 42 & 48 & 50 & 46 & 48 & 40 & 45 & 43 & 47 \\
\hline 2018 & 45 & 43 & 40 & 40 & 39 & 44 & 36 & 36 & & & & & \\
\hline \multicolumn{14}{|c|}{$\begin{array}{l}\text { 10. Diffusion index of output/input prices ratio, industry, actual (percent improving over 1-month spans) } \\
\text { 10. Диффуузный индекс отношения цен на выпускаемую и приобретаемую продукцию, } \\
\text { промышленность, фактические изменения (доля предприятий с улучшившимся за } 1 \text { месяц } \\
\text { отношением) }\end{array}$} \\
\hline 1992 & - & 42 & 44 & 41 & 24 & 31 & 28 & 28 & 29 & 27 & 29 & 32 & - \\
\hline 1993 & 28 & 31 & 28 & 23 & 25 & 22 & 21 & 25 & 28 & 21 & 24 & 26 & 25 \\
\hline 1994 & 21 & 24 & 18 & 28 & 23 & 25 & 23 & 31 & 27 & 2 & 23 & 30 & 25 \\
\hline 1995 & 25 & 27 & 29 & 30 & 28 & 29 & 29 & 33 & 33 & 32 & 27 & 33 & 30 \\
\hline 1996 & 29 & 35 & 32 & 33 & 34 & 36 & 35 & 34 & 36 & 3 & 36 & 39 & 34 \\
\hline 1997 & 39 & 36 & 37 & 38 & 39 & 41 & 39 & 40 & 42 & 44 & 42 & 40 & 40 \\
\hline 1998 & 41 & 41 & 39 & 42 & 40 & 41 & 44 & 35 & 23 & 2 & 27 & 26 & 35 \\
\hline 1999 & 27 & 30 & 29 & 31 & 32 & 31 & 32 & 31 & 31 & 3 & 33 & 35 & 31 \\
\hline 2000 & 30 & 36 & 35 & 36 & 33 & 35 & 34 & 37 & 30 & 36 & 35 & 37 & 34 \\
\hline 2001 & 35 & 32 & 36 & 40 & 37 & 38 & 41 & 40 & 41 & 41 & 41 & 41 & 39 \\
\hline 2002 & 40 & 36 & 40 & 43 & 37 & 37 & 38 & 39 & 38 & 38 & 39 & 37 & 38 \\
\hline 2003 & 33 & 3 & 32 & 37 & 37 & 40 & 4 & 4 & 39 & & 3 & 37 & 37 \\
\hline 2004 & 32 & 33 & 34 & 33 & 35 & 37 & 38 & 37 & 34 & 33 & 35 & 39 & 35 \\
\hline 2005 & 31 & 35 & 38 & 36 & 39 & 40 & 4 & 42 & 37 & 3 & 3 & 41 & 38 \\
\hline 2006 & 33 & 36 & 39 & 40 & 42 & 41 & 39 & 39 & 40 & 43 & 40 & 44 & 40 \\
\hline 2007 & 38 & 39 & 38 & 39 & 37 & 44 & 41 & 40 & 39 & 37 & 37 & 36 & 39 \\
\hline 2008 & 34 & 37 & 32 & 33 & 30 & 33 & 37 & 34 & 36 & 37 & 31 & 39 & 34 \\
\hline 2009 & 25 & 27 & 30 & 42 & 39 & 41 & 41 & 39 & 37 & 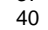 & 40 & 44 & 37 \\
\hline 2010 & 35 & 42 & 39 & 37 & 39 & 43 & 44 & 38 & 37 & 37 & 34 & 34 & 38 \\
\hline 2011 & 28 & 33 & 34 & 37 & 41 & 40 & 43 & 44 & 46 & 42 & 42 & 46 & 40 \\
\hline 2012 & 41 & 43 & 46 & 44 & 40 & 40 & 43 & 42 & 42 & 40 & 45 & 41 & 42 \\
\hline 2013 & 42 & 39 & 39 & 46 & 43 & 40 & 41 & 39 & 43 & 43 & 44 & 43 & 42 \\
\hline 2014 & 36 & 37 & 33 & 41 & 39 & 42 & 40 & 42 & 34 & 34 & 22 & 26 & 36 \\
\hline 2015 & 18 & 31 & 39 & 42 & 40 & 35 & 37 & 29 & 32 & 34 & 34 & 41 & 34 \\
\hline 2016 & 30 & 37 & 43 & 38 & 40 & 40 & 39 & 41 & 40 & 44 & 40 & 39 & 39 \\
\hline 2017 & 35 & 40 & 39 & 36 & 41 & 38 & 42 & 44 & 37 & 41 & 46 & 39 & 40 \\
\hline 2018 & 43 & 41 & 33 & 42 & 40 & 40 & 42 & 34 & & & & & \\
\hline
\end{tabular}




\begin{tabular}{|c|c|c|c|c|c|c|c|c|c|c|c|c|c|}
\hline Year & Jan. & Feb. & Mar. & Apr. & May & June & July & Aug. & Sept & Oct. & Nov. & Dec. & Annual \\
\hline \multicolumn{14}{|c|}{$\begin{array}{l}\text { 11. Diffusion index of credit terms, industry, actual (percent improving over 1-month spans) } \\
\text { 11. Дифффузный индекс условий получения банковских кредитов, промышленность, фактические } \\
\text { изменения (доля предприятий с улучшившимися за } 1 \text { месяц условиями) }\end{array}$} \\
\hline 1991 & - & - & - & - & - & - & - & - & - & - & - & 38 & - \\
\hline 1992 & 27 & 18 & 15 & 14 & 9 & 17 & 24 & 29 & 35 & 40 & 35 & 43 & 25 \\
\hline 1993 & 38 & 29 & 33 & 30 & 29 & 27 & 20 & 20 & 25 & 22 & 19 & 18 & 26 \\
\hline 1994 & 24 & 25 & 29 & 33 & 35 & 43 & 49 & 51 & 49 & 42 & 34 & 36 & 38 \\
\hline 1995 & 32 & 35 & 39 & 40 & 44 & 49 & 48 & 47 & 42 & 37 & 44 & 42 & 42 \\
\hline 1996 & 39 & 44 & 41 & 43 & 38 & 43 & 35 & 37 & 38 & 44 & 40 & 44 & 40 \\
\hline 1997 & 43 & 47 & 46 & 47 & 46 & 51 & 47 & 49 & 50 & 47 & 39 & 36 & 46 \\
\hline 1998 & 31 & 32 & 38 & 40 & 25 & 25 & 26 & 19 & 22 & 26 & 22 & 34 & 28 \\
\hline 1999 & 36 & 42 & 40 & 45 & 44 & 45 & 43 & 46 & 47 & 47 & 46 & 47 & 44 \\
\hline 2000 & 50 & 48 & 56 & 54 & 51 & 50 & 57 & 53 & 53 & 50 & 51 & 52 & 52 \\
\hline 2001 & 49 & 49 & 48 & 48 & 48 & 50 & 50 & 49 & 49 & 49 & 48 & 49 & 49 \\
\hline 2002 & 49 & 49 & 49 & 49 & 49 & 48 & 47 & 48 & 48 & 47 & 48 & 48 & 48 \\
\hline 2003 & 48 & 52 & 51 & 51 & 50 & 52 & 49 & 52 & 50 & 48 & 51 & 47 & 50 \\
\hline 2004 & 53 & 51 & 52 & 51 & 51 & 50 & 49 & 48 & 48 & 47 & 49 & 49 & 50 \\
\hline 2005 & 49 & 49 & 49 & 50 & 49 & 50 & 49 & 49 & 49 & 50 & 49 & 51 & 49 \\
\hline 2006 & 47 & 48 & 51 & 51 & 50 & 51 & 52 & 51 & 51 & 52 & 50 & 51 & 50 \\
\hline 2007 & 50 & 51 & 51 & 51 & 50 & 52 & 49 & 50 & 48 & 46 & 44 & 45 & 49 \\
\hline 2008 & 42 & 46 & 43 & 42 & 43 & 47 & 46 & 41 & 29 & 16 & 18 & 21 & 36 \\
\hline 2009 & 19 & 23 & 25 & 32 & 35 & 32 & 38 & 44 & 40 & 46 & 48 & 48 & 36 \\
\hline 2010 & 44 & 48 & 46 & 48 & 50 & 47 & 51 & 49 & 50 & 51 & 51 & 49 & 49 \\
\hline 2011 & - & - & - & - & - & - & - & - & - & - & - & - & - \\
\hline \multicolumn{14}{|c|}{$\begin{array}{c}\text { 14. Diffusion index of expenditures for equipment, industry, actual (percent rising over 1-month spans) } \\
\text { 14. Диффоззный индекс закупок оборудования, промышленность, фактические изменения (доля } \\
\text { предприятий с растущим за } 1 \text { месяц показателем) }\end{array}$} \\
\hline 1993 & 24 & 22 & 28 & 19 & 30 & 20 & 27 & 22 & 23 & 20 & 21 & 23 & 23 \\
\hline 1994 & 23 & 17 & 15 & 16 & 15 & 16 & 18 & 20 & 18 & 14 & 20 & 23 & 18 \\
\hline 1995 & 19 & 17 & 21 & 20 & 19 & 24 & 25 & 18 & 20 & 22 & 21 & 19 & 20 \\
\hline 1996 & 17 & 18 & 17 & 16 & 16 & 15 & 17 & 15 & 15 & 15 & 18 & 19 & 16 \\
\hline 1997 & 15 & 17 & 13 & 12 & 21 & 18 & 14 & 17 & 24 & 21 & 20 & 18 & 18 \\
\hline 1998 & 18 & 19 & 17 & 16 & 16 & 16 & 18 & 13 & 12 & 14 & 17 & 19 & 16 \\
\hline 1999 & 20 & 18 & 20 & 23 & 25 & 27 & 24 & 28 & 27 & 22 & 25 & 25 & 24 \\
\hline 2000 & 26 & 26 & 28 & 25 & 29 & 26 & 29 & 30 & 29 & 28 & 27 & 25 & 27 \\
\hline 2001 & 23 & 26 & 24 & 27 & 29 & 33 & 30 & 30 & 33 & 33 & 31 & 31 & 29 \\
\hline 2002 & 28 & 25 & 26 & 26 & 28 & 29 & 27 & 30 & 29 & 28 & 24 & 24 & 27 \\
\hline 2003 & 25 & 26 & 32 & 28 & 33 & 32 & 28 & 30 & 28 & 31 & 32 & 33 & 30 \\
\hline 2004 & 28 & 31 & 30 & 30 & 29 & 28 & 34 & 31 & 29 & 30 & 28 & 31 & 30 \\
\hline 2005 & 24 & 25 & 31 & 31 & 33 & 31 & 34 & 36 & 31 & 29 & 29 & 31 & 30 \\
\hline 2006 & 25 & 34 & 34 & 30 & 38 & 37 & 41 & 38 & 38 & 36 & 36 & 37 & 35 \\
\hline 2007 & 32 & 39 & 42 & 41 & 38 & 43 & 40 & 34 & 32 & 33 & 35 & 33 & 37 \\
\hline 2008 & 31 & 35 & 38 & 37 & 38 & 38 & 37 & 37 & 33 & 26 & 23 & 20 & 33 \\
\hline 2009 & 13 & 16 & 17 & 19 & 21 & 21 & 20 & 28 & 25 & 24 & 26 & 29 & 22 \\
\hline 2010 & 27 & 24 & 26 & 32 & 29 & 28 & 25 & 25 & 27 & 29 & 31 & 27 & 27 \\
\hline 2011 & 29 & 30 & 30 & 29 & 34 & 32 & 29 & 32 & 33 & 35 & 31 & 32 & 31 \\
\hline 2012 & 27 & 28 & 35 & 33 & 30 & 29 & 33 & 37 & 36 & 31 & 30 & 32 & 32 \\
\hline 2013 & 25 & 31 & 38 & 32 & 30 & 37 & 31 & 33 & 32 & 34 & 29 & 31 & 32 \\
\hline 2014 & 28 & 30 & 32 & 29 & 27 & 27 & 30 & 34 & 26 & 26 & 23 & 24 & 28 \\
\hline 2015 & 19 & 23 & 30 & 28 & 30 & 28 & 28 & 23 & 18 & 22 & 22 & 28 & 25 \\
\hline 2016 & 22 & 25 & 24 & 25 & 29 & 32 & 30 & 25 & 26 & 27 & 26 & 23 & 26 \\
\hline 2017 & 19 & 32 & 32 & 26 & 30 & 32 & 29 & 34 & 26 & 25 & 30 & 32 & 29 \\
\hline 2018 & 23 & 34 & 34 & 36 & 34 & 40 & 25 & 28 & & & & & \\
\hline
\end{tabular}




\begin{tabular}{|c|c|c|c|c|c|c|c|c|c|c|c|c|c|}
\hline Year & Jan. & Feb. & Mar. & Apr. & May & June & July & Aug. & Sept & Oct. & Nov. & Dec. & Annual \\
\hline \multicolumn{14}{|c|}{$\begin{array}{l}\text { 21. Diffusion index of output prices, industry, anticipated (percent rising over 3-month spans) } \\
\text { 21. Диффузный индекс цен на выпускаемую продукцию, промышленность, ожидаемые изменения } \\
\text { (доля предприятий с растущим за } 3 \text { месяца показателем) }\end{array}$} \\
\hline 1992 & - & - & - & - & - & - & 78 & 86 & 74 & 78 & 84 & 91 & - \\
\hline 1993 & 95 & 94 & 94 & 93 & 94 & 94 & 89 & 94 & 95 & 89 & 98 & 95 & 94 \\
\hline 1994 & 95 & 95 & 96 & 91 & 93 & 88 & 91 & 86 & 86 & 91 & 93 & 92 & 91 \\
\hline 1995 & 93 & 94 & 95 & 96 & 92 & 91 & 89 & 90 & 91 & 95 & 91 & 89 & 92 \\
\hline 1996 & 90 & 89 & 90 & 88 & 88 & 81 & 73 & 77 & 75 & 79 & 75 & 77 & 82 \\
\hline 1997 & 73 & 74 & 78 & 69 & 70 & 67 & 65 & 64 & 60 & 64 & 66 & 65 & 68 \\
\hline 1998 & 63 & 66 & 67 & 56 & 50 & 52 & 48 & 47 & 48 & 53 & 80 & 83 & 59 \\
\hline 1999 & 83 & 89 & 89 & 87 & 83 & 86 & 84 & 80 & 86 & 86 & 84 & 84 & 85 \\
\hline 2000 & 86 & 87 & 87 & 88 & 81 & 80 & 82 & 81 & 83 & 85 & 85 & 84 & 84 \\
\hline 2001 & 84 & 85 & 85 & 86 & 80 & 79 & 73 & 78 & 76 & 75 & 74 & 70 & 79 \\
\hline 2002 & 81 & 83 & 83 & 77 & 79 & 74 & 68 & 72 & 74 & 74 & 79 & 73 & 76 \\
\hline 2003 & 74 & 78 & 84 & 83 & 79 & 75 & 74 & 76 & 74 & 71 & 77 & 72 & 76 \\
\hline 2004 & 80 & 81 & 85 & 76 & 73 & 79 & 74 & 74 & 69 & 74 & 73 & 73 & 76 \\
\hline 2005 & 79 & 85 & 81 & 76 & 73 & 70 & 67 & 67 & 69 & 74 & 70 & 68 & 73 \\
\hline 2006 & 77 & 82 & 79 & 77 & 69 & 69 & 67 & 73 & 73 & 79 & 74 & 74 & 74 \\
\hline 2007 & 80 & 84 & 85 & 82 & 73 & 73 & 75 & 72 & 78 & 75 & 79 & 77 & 78 \\
\hline 2008 & 82 & 85 & 89 & 81 & 81 & 77 & 74 & 75 & 74 & 75 & 72 & 69 & 78 \\
\hline 2009 & 67 & 57 & 57 & 61 & 58 & 55 & 53 & 52 & 53 & 58 & 55 & 58 & 57 \\
\hline 2010 & 64 & 69 & 67 & 76 & 64 & 67 & 65 & 68 & 66 & 67 & 66 & 62 & 67 \\
\hline 2011 & 75 & 80 & 84 & 81 & 71 & 70 & 67 & 69 & 71 & 68 & 67 & 61 & 72 \\
\hline 2012 & 67 & 67 & 74 & 68 & 63 & 61 & 64 & 67 & 66 & 66 & 63 & 69 & 66 \\
\hline 2013 & 70 & 73 & 75 & 63 & 68 & 60 & 67 & 65 & 64 & 56 & 55 & 58 & 64 \\
\hline 2014 & 64 & 67 & 69 & 63 & 58 & 63 & 63 & 58 & 63 & 67 & 63 & 66 & 64 \\
\hline 2015 & 81 & 78 & 85 & 81 & 74 & 66 & 60 & 53 & 60 & 57 & 65 & 64 & 69 \\
\hline 2016 & 73 & 69 & 74 & 73 & 61 & 57 & 58 & 65 & 69 & 64 & 63 & 68 & 66 \\
\hline 2017 & 67 & 66 & 73 & 71 & 60 & 61 & 57 & 63 & 60 & 62 & 55 & 57 & 63 \\
\hline 2018 & 68 & 69 & 76 & 61 & 62 & 66 & 50 & 57 & 64 & 56 & 54 & & \\
\hline \multirow{2}{*}{\multicolumn{14}{|c|}{$\begin{array}{l}\text { 22. Diffusion index of input prices, industry, anticipated (percent rising over 3-month spans) } \\
\text { 22. Диффузный индекс цен на покупаемую продукцию, промышленность, ожидаемые изменения (доля } \\
\text { предприятий с растущим за } 3 \text { месяца показателем) } \\
\end{array}$}} \\
\hline & & & & & & & & & & & & & \\
\hline 1992 & - & - & - & - & - & - & 89 & 94 & 91 & 91 & 92 & 97 & - \\
\hline 1993 & 100 & 97 & 98 & 99 & 98 & 97 & 97 & 9 & 97 & 91 & $9 s$ & 99 & 97 \\
\hline 1994 & 99 & 97 & 99 & 98 & 99 & 95 & 99 & 98 & 95 & 98 & 97 & 98 & 98 \\
\hline 1995 & 99 & 99 & 95 & 98 & 99 & 97 & 98 & 97 & 97 & 99 & 96 & 95 & 97 \\
\hline 1996 & 95 & 95 & 98 & 96 & 96 & 95 & 92 & 93 & 92 & 92 & 92 & 93 & 94 \\
\hline 1997 & 93 & 92 & 90 & 90 & 88 & 88 & 85 & 82 & 83 & 86 & 84 & 85 & 87 \\
\hline 1998 & 83 & 82 & 87 & 78 & 69 & 70 & 64 & 70 & 64 & 67 & 9 & 96 & 77 \\
\hline 1999 & 96 & 97 & 97 & 96 & 95 & 95 & 96 & 94 & 97 & 95 & 9 & 95 & 96 \\
\hline 2000 & 97 & 95 & 9 & 96 & 93 & 94 & 95 & 95 & 95 & 95 & 92 & 95 & 95 \\
\hline 2001 & 94 & 96 & 9 & 95 & 92 & 92 & 88 & 9 & 90 & 88 & 8 & 89 & 92 \\
\hline 2002 & 92 & 93 & 92 & 94 & 90 & 88 & 89 & 91 & 90 & 93 & 93 & 90 & 91 \\
\hline 2003 & 93 & 95 & 98 & 95 & 94 & 92 & 9 & 8 & 90 & 91 & $8 \varepsilon$ & 91 & 92 \\
\hline 2004 & 93 & 94 & 94 & 93 & 91 & 90 & 84 & 87 & 86 & 86 & 89 & 89 & 90 \\
\hline 2005 & 95 & 95 & 93 & 90 & 90 & 85 & 8 & 82 & 86 & 89 & 8 & 88 & 88 \\
\hline 2006 & 91 & 94 & 92 & 91 & 86 & 87 & 81 & 8 & 83 & 91 & 88 & 90 & 88 \\
\hline 2007 & 91 & 93 & 95 & 92 & 84 & 88 & $8 \varepsilon$ & 85 & 85 & 88 & 84 & 89 & 89 \\
\hline 2008 & 92 & 96 & 95 & 91 & 92 & 87 & 8 & 8 & 90 & 89 & 88 & 85 & 90 \\
\hline 2009 & 79 & 71 & 69 & 78 & 82 & 73 & 75 & 74 & 75 & 81 & 81 & 82 & 77 \\
\hline 2010 & 86 & 89 & 86 & 86 & 81 & 84 & 84 & 8 & 85 & 85 & 8 & 81 & 84 \\
\hline 20 & 92 & 93 & 96 & 94 & 88 & 85 & 8 & 83 & 81 & 80 & 82 & 79 & 86 \\
\hline 201 & 85 & 82 & 84 & 82 & 78 & 77 & 85 & 82 & 82 & 84 & 8 & 81 & 82 \\
\hline 20 & 89 & 84 & 88 & 81 & 82 & 76 & 71 & 77 & 79 & 77 & 75 & 78 & 80 \\
\hline 2014 & 80 & 84 & 84 & 80 & 79 & 84 & 84 & 79 & 80 & 83 & 77 & 87 & 82 \\
\hline 20 & 92 & 95 & 9 & 93 & 90 & 80 & 73 & 77 & 81 & 80 & 89 & 84 & 85 \\
\hline 201 & 85 & 86 & $8 \varepsilon$ & 84 & 80 & 75 & 80 & 82 & 80 & 82 & 81 & 84 & 82 \\
\hline 2017 & 87 & 84 & 89 & 84 & 73 & 82 & 79 & 81 & 83 & 84 & 80 & 80 & 82 \\
\hline 2018 & 86 & 77 & 84 & 82 & 87 & 82 & 75 & 86 & 83 & 83 & 82 & & \\
\hline
\end{tabular}


The Russian Economic Barometer, Vol. XXVII, 3/4, 2018

\begin{tabular}{|c|c|c|c|c|c|c|c|c|c|c|c|c|c|}
\hline Year & Jan. & Feb. & Mar. & Apr. & May & June & July & Aug. & Sept & Oct. & Nov. & Dec. & Annual \\
\hline \multicolumn{14}{|c|}{$\begin{array}{l}\text { 24. Diffusion index of wages, industry, anticipated (percent rising over 3-month spans) } \\
\text { 24. Диффузный индекс заработной платы, промышленность, ожидаемые изменения } \\
\text { (доля предприятий с растущим за } 3 \text { месяца показателем) }\end{array}$} \\
\hline 1992 & - & - & - & - & - & - & 72 & 78 & 76 & 83 & 82 & 85 & - \\
\hline 1993 & 85 & 84 & 88 & 87 & 87 & 94 & 91 & 91 & 92 & 84 & 93 & 87 & 86 \\
\hline 1994 & 85 & 91 & 89 & 82 & 80 & 77 & 77 & 74 & 77 & 78 & 75 & 80 & 80 \\
\hline 1995 & 83 & 86 & 90 & 86 & 84 & 84 & 87 & 83 & 85 & 87 & 84 & 79 & 85 \\
\hline 1996 & 81 & 77 & 75 & 71 & 76 & 70 & 66 & 66 & 64 & 58 & 56 & 54 & 68 \\
\hline 1997 & 54 & 55 & 61 & 58 & 58 & 60 & 62 & 56 & 58 & 55 & 55 & 56 & 57 \\
\hline 1998 & 47 & 55 & 53 & 55 & 50 & 53 & 49 & 44 & 46 & 43 & 51 & 51 & 50 \\
\hline 1999 & 60 & 63 & 68 & 70 & 73 & 74 & 68 & 71 & 74 & 72 & 72 & 73 & 70 \\
\hline 2000 & 69 & 72 & 72 & 78 & 72 & 71 & 77 & 73 & 75 & 76 & 75 & 72 & 74 \\
\hline 2001 & 76 & 73 & 73 & 76 & 71 & 77 & 72 & 73 & 75 & 72 & 71 & 68 & 73 \\
\hline 2002 & 69 & 71 & 74 & 72 & 75 & 72 & 70 & 72 & 72 & 69 & 70 & 67 & 71 \\
\hline 2003 & 61 & 66 & 72 & 70 & 76 & 73 & 74 & 68 & 76 & 70 & 65 & 65 & 70 \\
\hline 2004 & 64 & 66 & 68 & 70 & 71 & 77 & 72 & 73 & 70 & 66 & 63 & 61 & 68 \\
\hline 2005 & 64 & 70 & 74 & 75 & 75 & 72 & 73 & 73 & 69 & 71 & 65 & 64 & 70 \\
\hline 2006 & 62 & 63 & 68 & 73 & 76 & 72 & 74 & 76 & 74 & 73 & 71 & 69 & 71 \\
\hline 2007 & 62 & 71 & 75 & 80 & 76 & 76 & 80 & 79 & 73 & 74 & 70 & 68 & 74 \\
\hline 2008 & 68 & 71 & 73 & 77 & 77 & 77 & 79 & 77 & 76 & 70 & 70 & 63 & 73 \\
\hline 2009 & 52 & 39 & 41 & 46 & 38 & 50 & 49 & 49 & 47 & 52 & 46 & 45 & 46 \\
\hline 2010 & 44 & 47 & 50 & 67 & 62 & 64 & 65 & 63 & 61 & 59 & 58 & 54 & 58 \\
\hline 2011 & 51 & 50 & 57 & 67 & 65 & 67 & 65 & 66 & 65 & 57 & 58 & 51 & 60 \\
\hline 2012 & 51 & 55 & 62 & 66 & 66 & 65 & 69 & 67 & 62 & 57 & 62 & 58 & 62 \\
\hline 2013 & 53 & 56 & 62 & 71 & 63 & 62 & 60 & 60 & 57 & 61 & 61 & 52 & 60 \\
\hline 2014 & 51 & 56 & 56 & 58 & 60 & 61 & 62 & 63 & 63 & 54 & 57 & 49 & 58 \\
\hline 2015 & 48 & 49 & 57 & 61 & 56 & 70 & 62 & 61 & 55 & 56 & 55 & 46 & 56 \\
\hline 2016 & 54 & 45 & 47 & 54 & 59 & 53 & 56 & 58 & 56 & 57 & 51 & 60 & 54 \\
\hline 2017 & 52 & 45 & 55 & 63 & 61 & 65 & 57 & 57 & 59 & 50 & 48 & 49 & 55 \\
\hline 2018 & 50 & 52 & 52 & 63 & 61 & 62 & 55 & 62 & 58 & 50 & 43 & & \\
\hline \multicolumn{14}{|c|}{$\begin{array}{l}\text { 25. Diffusion index of employment, industry, anticipated (percent rising over 3-month spans) } \\
\text { 25. Дифрфузый индекс занятости, промышленность, ожидаемые изменения } \\
\text { (доля предприятий с растущим за } 3 \text { месяца показателем) }\end{array}$} \\
\hline 1992 & - & - & - & - & - & - & 34 & 28 & 27 & 25 & 22 & 25 & - \\
\hline 1993 & 24 & 22 & 32 & 30 & 32 & 33 & 33 & 36 & 34 & 34 & 33 & 33 & 31 \\
\hline 1994 & 27 & 26 & 27 & 24 & 25 & 23 & 22 & 19 & 19 & 20 & 19 & 20 & 23 \\
\hline 1995 & 25 & 28 & 27 & 32 & 32 & 34 & 34 & 32 & 34 & 35 & 33 & 30 & 31 \\
\hline 1996 & 29 & 27 & 28 & 28 & 30 & 24 & 27 & 25 & 25 & 23 & 21 & 23 & 26 \\
\hline 1997 & 21 & 25 & 22 & 25 & 26 & 33 & 35 & 32 & 33 & 30 & 33 & 34 & 29 \\
\hline 1998 & 26 & 30 & 34 & 38 & 39 & 34 & 37 & 29 & 31 & 29 & 28 & 30 & 32 \\
\hline 1999 & 30 & 34 & 35 & 39 & 45 & 42 & 45 & 45 & 48 & 43 & 48 & 47 & 42 \\
\hline 2000 & 46 & 49 & 50 & 48 & 52 & 52 & 50 & 52 & 51 & 4 & 50 & 53 & 50 \\
\hline 2001 & 47 & 49 & 51 & 53 & 50 & 49 & 47 & 48 & 45 & 48 & 44 & 44 & 48 \\
\hline 2002 & 47 & 48 & 44 & 49 & 47 & 47 & 42 & 40 & 40 & 39 & 41 & 13 & 44 \\
\hline 2003 & 40 & 37 & 45 & 40 & 43 & 43 & 43 & 44 & 44 & 42 & 40 & 39 & 42 \\
\hline 2004 & 43 & 37 & 40 & 44 & 38 & 43 & 39 & 43 & 44 & 40 & 39 & & 41 \\
\hline 2005 & 37 & 39 & 42 & 41 & 41 & 41 & 43 & 43 & 41 & 41 & 37 & 33 & 40 \\
\hline 2006 & 35 & 38 & 40 & 45 & 45 & 43 & 44 & 45 & 43 & 42 & 45 & 44 & 42 \\
\hline 2007 & 41 & 47 & 51 & 52 & 51 & 50 & 53 & 52 & 47 & 50 & 46 & 43 & 49 \\
\hline 2008 & 49 & 48 & 51 & 54 & 54 & 51 & 49 & 48 & 43 & 47 & 44 & 38 & 48 \\
\hline 2009 & 31 & 24 & 26 & 33 & 28 & 30 & 38 & 33 & 38 & 32 & 33 & 34 & 32 \\
\hline 2010 & 32 & 38 & 38 & 42 & 42 & 48 & 47 & 55 & 44 & 39 & 41 & 42 & 42 \\
\hline 2011 & 43 & 42 & 47 & 50 & 46 & 51 & 48 & 53 & 51 & 48 & 51 & 42 & 48 \\
\hline 2012 & 45 & 44 & 48 & 54 & 52 & 54 & 56 & 49 & 41 & 49 & 54 & 47 & 49 \\
\hline 2013 & 45 & 44 & 47 & 51 & 53 & 49 & 49 & 45 & 37 & 43 & 42 & 40 & 45 \\
\hline 2014 & 42 & 39 & 43 & 45 & 45 & 45 & 42 & 41 & 38 & 40 & 45 & 40 & 42 \\
\hline 2015 & 38 & 36 & 38 & 39 & 43 & 48 & 48 & 47 & 48 & 39 & 40 & 32 & 41 \\
\hline 2016 & 36 & 28 & 35 & 40 & 46 & 43 & 40 & 38 & 48 & 41 & 49 & 43 & 41 \\
\hline 2017 & 39 & 38 & 53 & 46 & 49 & 48 & 49 & 44 & 46 & 47 & 42 & 42 & 45 \\
\hline 2018 & 40 & 41 & 48 & 53 & 54 & 49 & 45 & 43 & 51 & 33 & 39 & & \\
\hline
\end{tabular}




\begin{tabular}{|c|c|c|c|c|c|c|c|c|c|c|c|c|c|}
\hline Year & Jan. & Feb. & Mar. & Apr. & May & June & July & Aug. & Sept & Oct. & Nov. & Dec. & Annual \\
\hline \multicolumn{14}{|c|}{$\begin{array}{l}\text { 26. Diffusion index of output, industry, anticipated (percent rising over 3-month spans) } \\
\text { 26. Диффузный индекс выпуска, промышленность, ожидаемые изменения } \\
\text { (доля предприятий с растущим за } 3 \text { месяца показателем) }\end{array}$} \\
\hline 1992 & - & - & - & - & - & - & 39 & 33 & 38 & 32 & 33 & 30 & - \\
\hline 1993 & 34 & 37 & 47 & 46 & 48 & 48 & 45 & 46 & 49 & 40 & 37 & 42 & 43 \\
\hline 1994 & 36 & 33 & 33 & 37 & 39 & 29 & 37 & 35 & 33 & 37 & 36 & 30 & 36 \\
\hline 1995 & 31 & 41 & 40 & 48 & 46 & 47 & 48 & 42 & 47 & 43 & 44 & 44 & 43 \\
\hline 1996 & 34 & 37 & 39 & 42 & 45 & 37 & 42 & 41 & 40 & 33 & 30 & 27 & 37 \\
\hline 1997 & 30 & 34 & 33 & 43 & 44 & 47 & 46 & 47 & 48 & 40 & 46 & 41 & 42 \\
\hline 1998 & 31 & 43 & 49 & 55 & 48 & 44 & 45 & 49 & 41 & 44 & 29 & 33 & 43 \\
\hline 1999 & 37 & 40 & 47 & 55 & 53 & 48 & 54 & 52 & 56 & 50 & 49 & 55 & 50 \\
\hline 2000 & 49 & 51 & 57 & 63 & 56 & 54 & 56 & 62 & 54 & 60 & 56 & 58 & 56 \\
\hline 2001 & 46 & 53 & 57 & 66 & 59 & 61 & 59 & 58 & 53 & 55 & 51 & 48 & 56 \\
\hline 2002 & 47 & 46 & 57 & 61 & 58 & 57 & 54 & 57 & 60 & 48 & 52 & 48 & 54 \\
\hline 2003 & 44 & 49 & 56 & 66 & 66 & 64 & 61 & 59 & 59 & 58 & 52 & 49 & 57 \\
\hline 2004 & 44 & 43 & 57 & 63 & 59 & 65 & 61 & 65 & 62 & 57 & 53 & 44 & 56 \\
\hline 2005 & 44 & 51 & 55 & 67 & 66 & 61 & 62 & 59 & 57 & 60 & 53 & 47 & 57 \\
\hline 2006 & 45 & 47 & 60 & 68 & 67 & 66 & 65 & 71 & 63 & 54 & 53 & 56 & 60 \\
\hline 2007 & 46 & 52 & 58 & 73 & 66 & 68 & 69 & 68 & 65 & 54 & 52 & 46 & 60 \\
\hline 2008 & 45 & 49 & 55 & 68 & 70 & 64 & 64 & 60 & 61 & 52 & 50 & 39 & 56 \\
\hline 2009 & 28 & 21 & 27 & 43 & 36 & 46 & 44 & 41 & 45 & 44 & 40 & 38 & 38 \\
\hline 2010 & 23 & 34 & 43 & 68 & 55 & 59 & 57 & 57 & 56 & 45 & 45 & 47 & 49 \\
\hline 2011 & 32 & 32 & 50 & 68 & 65 & 57 & 63 & 61 & 57 & 54 & 54 & 50 & 54 \\
\hline 2012 & 38 & 40 & 49 & 72 & 67 & 66 & 66 & 58 & 56 & 48 & 58 & 47 & 55 \\
\hline 2013 & 34 & 44 & 61 & 66 & 63 & 62 & 54 & 55 & 51 & 50 & 46 & 43 & 52 \\
\hline 2014 & 29 & 43 & 44 & 56 & 56 & 63 & 55 & 55 & 56 & 48 & 45 & 45 & 50 \\
\hline 2015 & 31 & 32 & 38 & 47 & 48 & 58 & 51 & 52 & 46 & 47 & 40 & 34 & 44 \\
\hline 2016 & 29 & 27 & 41 & 55 & 55 & 49 & 51 & 55 & 51 & 43 & 45 & 47 & 46 \\
\hline 2017 & 33 & 30 & 49 & 59 & 57 & 58 & 48 & 49 & 41 & 57 & 39 & 39 & 47 \\
\hline 2018 & 40 & 35 & 44 & 58 & 54 & 53 & 43 & 57 & 51 & 45 & 42 & & \\
\hline \multirow{2}{*}{\multicolumn{14}{|c|}{$\begin{array}{l}\text { 27. Diffusion index of expenditures for equipment, industry, anticipated (percent rising over 3-month spans) } \\
\text { 27. Диффрузный индекс закупок оборудования, промышленность, ожидаемые изменения } \\
\text { (доля предприятий с растущим за } 3 \text { месяца показателем) }\end{array}$}} \\
\hline & & & & & & & & & & & & & \\
\hline 1993 & - & - & - & 32 & 28 & 33 & 28 & 33 & 22 & 28 & 24 & 25 & - \\
\hline 1994 & 20 & 25 & 22 & 21 & 20 & 19 & 17 & 21 & 19 & 20 & 25 & 19 & 21 \\
\hline 1995 & 20 & 23 & 24 & 25 & 22 & 31 & 28 & 26 & 27 & 23 & 19 & 22 & 24 \\
\hline 1996 & 23 & 24 & 22 & 26 & 25 & 25 & 18 & 19 & 19 & 21 & 14 & 14 & 21 \\
\hline 1997 & 17 & 17 & 17 & 18 & 21 & 20 & 17 & 19 & 23 & 23 & 20 & 24 & 20 \\
\hline 1998 & 23 & 22 & 26 & 25 & 23 & 22 & 21 & 19 & 21 & 21 & 13 & 14 & 21 \\
\hline 1999 & 20 & 19 & 22 & 28 & 26 & 28 & 26 & 29 & 29 & 27 & 27 & 28 & 26 \\
\hline 2000 & 28 & 29 & 31 & 32 & 32 & 34 & 30 & 35 & 35 & 29 & 30 & 32 & 31 \\
\hline 2001 & 34 & 31 & 32 & 33 & 33 & 34 & 33 & 32 & 34 & 35 & 33 & 34 & 33 \\
\hline 2002 & 31 & 32 & 32 & 34 & 31 & 36 & 31 & 33 & 33 & 28 & 34 & 37 & 33 \\
\hline 2003 & 28 & 30 & 29 & 38 & 33 & 36 & 32 & 3 & 34 & 36 & 31 & 33 & 33 \\
\hline 2004 & 34 & 26 & 38 & 37 & 37 & 38 & 35 & 37 & 38 & 32 & 36 & 28 & 35 \\
\hline 2005 & 31 & 31 & 36 & 39 & 39 & 34 & 33 & 36 & 31 & 32 & 31 & 32 & 34 \\
\hline 2006 & 31 & 33 & 35 & 35 & 39 & 42 & 40 & 44 & 45 & 42 & 39 & 42 & 39 \\
\hline 2007 & 38 & 40 & 43 & 45 & 46 & 48 & 47 & 44 & 44 & 40 & 39 & 36 & 42 \\
\hline 2008 & 39 & 34 & 40 & 44 & 46 & 44 & 41 & 46 & 40 & 37 & 38 & 31 & 40 \\
\hline 2009 & 19 & 20 & 18 & 22 & 23 & 21 & 24 & 28 & 26 & 25 & 28 & 26 & 23 \\
\hline 2010 & 26 & 27 & 31 & 35 & 32 & 28 & 33 & 33 & 34 & 30 & 30 & 32 & 31 \\
\hline 2011 & 28 & 28 & 27 & 41 & 35 & 33 & 35 & 37 & 38 & 36 & 29 & 34 & 33 \\
\hline 2012 & 33 & 34 & 33 & 33 & 35 & 34 & 35 & 37 & 37 & 43 & 45 & 36 & 36 \\
\hline 2013 & 30 & 34 & 37 & 37 & 37 & 48 & 35 & 37 & 36 & 30 & 41 & 39 & 37 \\
\hline 2014 & 35 & 35 & 39 & 40 & 36 & 34 & 36 & 32 & 34 & 36 & 42 & 27 & 36 \\
\hline 201 & 30 & 22 & 30 & 27 & 28 & 35 & 30 & 26 & 27 & 26 & 29 & 18 & 27 \\
\hline 2016 & 24 & 19 & 28 & 34 & 29 & 29 & 27 & 31 & 35 & 33 & 22 & 28 & 28 \\
\hline 2017 & 27 & 33 & 33 & 29 & 36 & 41 & 25 & 27 & 31 & 30 & 33 & 39 & 32 \\
\hline 2018 & 33 & 34 & 32 & 35 & 44 & 42 & 35 & 38 & 38 & 28 & 29 & & \\
\hline
\end{tabular}


The Russian Economic Barometer, Vol. XXVII, 3/4, 2018

\begin{tabular}{|c|c|c|c|c|c|c|c|c|c|c|c|c|c|}
\hline Year & Jan. & Feb. & Mar. & Apr. & May & June & July & Aug. & Sept & Oct. & Nov. & Dec. & Annual \\
\hline \multicolumn{14}{|c|}{$\begin{array}{l}\text { 28. Diffusion index of financial situation, industry, anticipated (percent improving over 3-month spans) } \\
\text { 28. Диффузный индекс финансового состояния, промышленность, ожидаемые изменения (доля } \\
\text { предприятий, у которых оно улучшится через } 3 \text { месяца) }\end{array}$} \\
\hline 1993 & - & - & - & - & 45 & 42 & 41 & 40 & 36 & 38 & 43 & 43 & - \\
\hline 1994 & 30 & 34 & 38 & 36 & 32 & 33 & 29 & 33 & 32 & 29 & 41 & 35 & 34 \\
\hline 1995 & 32 & 31 & 39 & 39 & 36 & 41 & 42 & 40 & 42 & 40 & 38 & 32 & 38 \\
\hline 1996 & 38 & 33 & 38 & 38 & 41 & 38 & 39 & 41 & 37 & 29 & 28 & 26 & 36 \\
\hline 1997 & 27 & 31 & 36 & 39 & 41 & 39 & 42 & 40 & 43 & 37 & 38 & 40 & 38 \\
\hline 1998 & 31 & 34 & 41 & 44 & 45 & 43 & 45 & 38 & 40 & 33 & 24 & 28 & 37 \\
\hline 1999 & 34 & 36 & 41 & 44 & 46 & 47 & 48 & 48 & 50 & 47 & 43 & 43 & 44 \\
\hline 2000 & 43 & 49 & 54 & 52 & 54 & 57 & 56 & 55 & 55 & 53 & 49 & 44 & 52 \\
\hline 2001 & 47 & 52 & 54 & 60 & 55 & 59 & 60 & 60 & 56 & 50 & 52 & 50 & 55 \\
\hline 2002 & 49 & 53 & 54 & 60 & 57 & 59 & 58 & 57 & 61 & 54 & 51 & 50 & 55 \\
\hline 2003 & 47 & 53 & 51 & 59 & 63 & 60 & 57 & 58 & 63 & 57 & 52 & 51 & 56 \\
\hline 2004 & 48 & 51 & 53 & 64 & 60 & 63 & 58 & 60 & 56 & 56 & 53 & 49 & 56 \\
\hline 2005 & 46 & 52 & 58 & 69 & 66 & 61 & 61 & 64 & 58 & 61 & 57 & 48 & 58 \\
\hline 2006 & 51 & 52 & 56 & 61 & 62 & 62 & 65 & 64 & 63 & 59 & 58 & 57 & 59 \\
\hline 2007 & 50 & 47 & 56 & 66 & 64 & 65 & 68 & 65 & 66 & 61 & 53 & 55 & 60 \\
\hline 2008 & 48 & 52 & 56 & 61 & 68 & 64 & 63 & 60 & 57 & 57 & 53 & 40 & 57 \\
\hline 2009 & 34 & 29 & 37 & 50 & 47 & 51 & 44 & 48 & 52 & 47 & 40 & 38 & 43 \\
\hline 2010 & 37 & 42 & 52 & 65 & 58 & 63 & 62 & 63 & 58 & 51 & 52 & 46 & 54 \\
\hline 2011 & 41 & 42 & 49 & 67 & 65 & 58 & 63 & 60 & 60 & 55 & 54 & 52 & 55 \\
\hline 2012 & 43 & 53 & 58 & 64 & 65 & 66 & 69 & 63 & 58 & 54 & 58 & 53 & 59 \\
\hline 2013 & 39 & 47 & 49 & 55 & 58 & 59 & 70 & 57 & 55 & 51 & 44 & 42 & 52 \\
\hline 2014 & 47 & 42 & 49 & 53 & 58 & 58 & 56 & 55 & 61 & 51 & 57 & 50 & 53 \\
\hline 2015 & 36 & 38 & 40 & 43 & 55 & 61 & 57 & 54 & 51 & 50 & 45 & 40 & 48 \\
\hline 2016 & 43 & 36 & 43 & 53 & 55 & 53 & 51 & 50 & 46 & 44 & 43 & 46 & 47 \\
\hline 2017 & 46 & 47 & 46 & 51 & 54 & 48 & 48 & 49 & 49 & 46 & 49 & 53 & 49 \\
\hline 2018 & 44 & 45 & 55 & 60 & 57 & 55 & 47 & 55 & 57 & 53 & 46 & & \\
\hline \multicolumn{14}{|c|}{$\begin{array}{l}\text { 29. Diffusion index of order-book level, industry, anticipated (percent rising over 3-month spans } \\
\text { 29. Диффрузный индекс портфеля заказов, промышленность, ожидаемые изменения }\end{array}$} \\
\hline 1994 & - & - & - & 48 & 50 & 46 & 47 & 42 & 47 & 47 & 45 & 45 & - \\
\hline 1995 & 45 & 49 & 52 & 50 & 56 & 53 & 51 & 47 & 51 & 47 & 45 & 45 & 49 \\
\hline 1996 & 43 & 46 & 53 & 55 & 56 & 53 & 52 & 50 & 49 & 39 & 40 & 38 & 48 \\
\hline 1997 & 39 & 40 & 48 & 54 & 56 & 55 & 55 & 51 & 50 & 45 & 45 & 47 & 49 \\
\hline 1998 & 37 & 48 & 54 & 61 & 54 & 59 & 54 & 51 & 50 & 49 & 33 & 36 & 49 \\
\hline 1999 & 46 & 47 & 55 & 63 & 59 & 56 & 59 & 58 & 56 & 57 & 51 & 51 & 55 \\
\hline 2000 & 52 & 56 & 62 & 66 & 60 & 62 & 63 & 61 & 58 & 57 & 54 & 53 & 59 \\
\hline 2001 & 52 & 59 & 62 & 69 & 61 & 64 & 61 & 63 & 61 & 59 & 56 & 51 & 60 \\
\hline 2002 & 54 & 53 & 62 & 68 & 66 & 62 & 64 & 66 & 65 & 58 & 54 & 51 & 60 \\
\hline 2003 & 44 & 56 & 61 & 69 & 68 & 67 & 61 & 65 & 66 & 60 & 53 & 49 & 60 \\
\hline 2004 & 46 & 56 & 65 & 72 & 65 & 68 & 63 & 71 & 62 & 56 & 51 & 50 & 60 \\
\hline 2005 & 45 & 51 & 62 & 75 & 73 & 64 & 65 & 63 & 62 & 61 & 51 & 45 & 60 \\
\hline 2006 & 52 & 51 & 63 & 68 & 71 & 64 & 65 & 64 & 63 & 57 & 50 & 55 & 60 \\
\hline 2007 & 44 & 54 & 65 & 76 & 70 & 71 & 69 & 67 & 67 & 57 & 55 & 48 & 62 \\
\hline 2008 & 48 & 49 & 65 & 75 & 73 & 64 & 68 & 65 & 59 & 58 & 55 & 40 & 60 \\
\hline 2009 & 33 & 32 & 48 & 56 & 55 & 57 & 55 & 52 & 55 & 47 & 44 & 39 & 48 \\
\hline 2010 & 32 & 44 & 61 & 72 & 58 & 67 & 66 & 67 & 62 & 48 & 48 & 44 & 56 \\
\hline 2011 & 40 & 40 & 53 & 73 & 64 & 60 & 64 & 60 & 65 & 57 & 58 & 48 & 57 \\
\hline 2012 & 34 & 48 & 60 & 68 & 71 & 64 & 65 & 65 & 62 & 55 & 54 & 46 & 58 \\
\hline 2013 & 40 & 45 & 64 & 66 & 67 & 59 & 65 & 59 & 60 & 48 & 44 & 42 & 55 \\
\hline 2014 & 44 & 47 & 51 & 65 & 64 & 60 & 61 & 58 & 62 & 56 & 52 & 45 & 55 \\
\hline 2015 & 41 & 42 & 45 & 54 & 58 & 64 & 59 & 55 & 56 & 52 & 52 & 45 & 52 \\
\hline 2016 & 43 & 37 & 48 & 61 & 62 & 59 & 60 & 56 & 57 & 48 & 47 & 49 & 52 \\
\hline 2017 & 43 & 42 & 60 & 65 & 66 & 60 & 50 & 58 & 53 & 53 & 47 & 46 & 54 \\
\hline 2018 & 41 & 45 & 58 & 66 & 58 & 59 & 57 & 54 & 60 & 44 & 40 & & \\
\hline
\end{tabular}




\begin{tabular}{|c|c|c|c|c|c|c|c|c|c|c|c|c|c|}
\hline Year & Jan. & Feb. & Mar. & Apr. & May & June & July & Aug. & Sept & Oct. & Nov. & Dec. & Annual \\
\hline \multicolumn{14}{|c|}{$\begin{array}{l}\text { 30. Diffusion index of indebtedness to banks, industry, anticipated (percent rising over 3-month spans) } \\
\text { 30. Дифрфузный индекс задолженности банкам, промышленность, ожидаемые изменения (доля } \\
\text { предприятий с растущим за } 3 \text { месяца показателем) }\end{array}$} \\
\hline 1994 & - & - & - & 50 & 44 & 45 & 48 & 47 & 53 & 44 & 40 & 41 & - \\
\hline 1995 & 45 & 44 & 40 & 42 & 35 & 37 & 36 & 34 & 36 & 31 & 36 & 35 & 38 \\
\hline 1996 & 33 & 37 & 39 & 43 & 40 & 41 & 33 & 36 & 36 & 40 & 40 & 38 & 38 \\
\hline 1997 & 39 & 43 & 32 & 35 & 34 & 36 & 32 & 31 & 31 & 26 & 29 & 31 & 33 \\
\hline 1998 & 33 & 31 & 30 & 29 & 30 & 31 & 23 & 27 & 26 & 29 & 27 & 21 & 28 \\
\hline 1999 & 23 & 22 & 18 & 21 & 23 & 20 & 18 & 19 & 23 & 18 & 17 & 18 & 20 \\
\hline 2000 & 22 & 24 & 17 & 19 & 19 & 24 & 19 & 20 & 17 & 19 & 18 & 19 & 20 \\
\hline 2001 & 17 & 20 & 22 & 16 & 19 & 25 & 23 & 22 & 21 & 21 & 22 & 24 & 21 \\
\hline 2002 & 21 & 26 & 20 & 22 & 29 & 21 & 24 & 24 & 16 & 23 & 20 & 19 & 22 \\
\hline 2003 & 22 & 18 & 25 & 23 & 23 & 22 & 23 & 26 & 20 & 26 & 24 & 23 & 23 \\
\hline 2004 & 25 & 29 & 27 & 23 & 27 & 19 & 18 & 27 & 22 & 22 & 23 & 23 & 24 \\
\hline 2005 & 35 & 25 & 24 & 26 & 28 & 28 & 27 & 25 & 26 & 21 & 26 & 26 & 26 \\
\hline 2006 & 25 & 30 & 26 & 22 & 24 & 28 & 24 & 27 & 28 & 23 & 29 & 29 & 26 \\
\hline 2007 & 30 & 29 & 30 & 26 & 26 & 24 & 25 & 26 & 25 & 25 & 21 & 26 & 26 \\
\hline 2008 & 27 & 26 & 23 & 26 & 27 & 28 & 22 & 23 & 24 & 26 & 30 & 26 & 26 \\
\hline 2009 & 35 & 27 & 32 & 34 & 29 & 26 & 27 & 23 & 23 & 24 & 30 & 23 & 28 \\
\hline 2010 & 22 & 26 & 21 & 22 & 29 & 22 & 22 & 23 & 24 & 23 & 20 & 17 & 23 \\
\hline 2011 & 24 & 29 & 23 & 26 & 25 & 26 & 28 & 23 & 18 & 25 & 20 & 24 & 24 \\
\hline 2012 & 23 & 20 & 19 & 21 & 20 & 21 & 19 & 23 & 19 & 23 & 22 & 18 & 21 \\
\hline 2013 & 24 & 29 & 25 & 25 & 27 & 27 & 20 & 29 & 26 & 24 & 25 & 27 & 26 \\
\hline 2014 & 23 & 23 & 24 & 32 & 27 & 16 & 30 & 24 & 26 & 23 & 19 & 24 & 24 \\
\hline 2015 & 26 & 28 & 24 & 27 & 21 & 23 & 24 & 28 & 30 & 15 & 24 & 29 & 25 \\
\hline 2016 & 21 & 24 & 29 & 32 & 24 & 21 & 30 & 30 & 26 & 27 & 29 & 22 & 26 \\
\hline 2017 & 24 & 25 & 29 & 16 & 32 & 30 & 30 & 23 & 28 & 24 & 28 & 21 & 26 \\
\hline 2018 & 18 & 26 & 15 & 17 & 23 & 20 & 22 & 17 & 18 & 26 & 27 & & \\
\hline \multicolumn{14}{|c|}{$\begin{array}{l}\text { 13. Capacity utilization rate, industry (normal monthly level }=100 \text { ) } \\
\text { 13. Загрузка производственных мощностей, промышленность } \\
\text { (нормальный месячный уровень }=100 \text { ) }\end{array}$} \\
\hline 1991 & - & - & - & - & - & - & - & - & - & - & - & 78 & - \\
\hline 1992 & 70 & 64 & 70 & 77 & 79 & 75 & 71 & 70 & 75 & 72 & 77 & 75 & 73 \\
\hline 1993 & 75 & 76 & 78 & 76 & 76 & 72 & 73 & 74 & 72 & 72 & 71 & 69 & 74 \\
\hline 1994 & 67 & 67 & 63 & 61 & 58 & 55 & 57 & 61 & 61 & 62 & 61 & 64 & 61 \\
\hline 1995 & 59 & 60 & 63 & 62 & 60 & 59 & 62 & 58 & 61 & 61 & 61 & 57 & 60 \\
\hline 1996 & 56 & 54 & 55 & 56 & 54 & 56 & 57 & 51 & 55 & 52 & 54 & 54 & 54 \\
\hline 1997 & 52 & 50 & 51 & 51 & 55 & 54 & 53 & 57 & 57 & 57 & 57 & 60 & 54 \\
\hline 1998 & 55 & 55 & 57 & 55 & 53 & 55 & 53 & 53 & 53 & 55 & 57 & 57 & 55 \\
\hline 1999 & 56 & 58 & 60 & 61 & 59 & 64 & 63 & 63 & 63 & 64 & 64 & 65 & 62 \\
\hline 2000 & 64 & 64 & 66 & 66 & 69 & 68 & 67 & 68 & 69 & 65 & 66 & 66 & 66 \\
\hline 2001 & 68 & 67 & 67 & 67 & 67 & 69 & 71 & 70 & 71 & 73 & 70 & 68 & 69 \\
\hline 2002 & 66 & 67 & 69 & 69 & 68 & 69 & 68 & 73 & 74 & 73 & 72 & 71 & 70 \\
\hline 2003 & 71 & 72 & 71 & 72 & 73 & 73 & 68 & 73 & 73 & 74 & 75 & 76 & 73 \\
\hline 2004 & 70 & 72 & 73 & 76 & 74 & 73 & 75 & 73 & 73 & 76 & 75 & 77 & 74 \\
\hline 2005 & 71 & 73 & 75 & 73 & 74 & 77 & 76 & 78 & 80 & 79 & 74 & 79 & 76 \\
\hline 2006 & 70 & 74 & 78 & 78 & 81 & 76 & 81 & 82 & 80 & 81 & 79 & 81 & 78 \\
\hline 2007 & 78 & 79 & 79 & 78 & 81 & 80 & 78 & 81 & 78 & 81 & 82 & 80 & 80 \\
\hline 2008 & 78 & 79 & 81 & 82 & 78 & 79 & 79 & 77 & 78 & 78 & 67 & 64 & 77 \\
\hline 2009 & 61 & 63 & 62 & 63 & 62 & 64 & 66 & 67 & 69 & 67 & 70 & 70 & 65 \\
\hline 2010 & 68 & 71 & 69 & 70 & 72 & 72 & 74 & 73 & 73 & 77 & 75 & 75 & 72 \\
\hline 2011 & 72 & 75 & 78 & 79 & 79 & 77 & 80 & 82 & 77 & 79 & 82 & 79 & 78 \\
\hline 2012 & 75 & 78 & 81 & 77 & 80 & 77 & 81 & 81 & 79 & 82 & 79 & 81 & 79 \\
\hline 2013 & 79 & 81 & 81 & 75 & 75 & 78 & 75 & 76 & 76 & 78 & 79 & 78 & 78 \\
\hline 2014 & 76 & 75 & 77 & 78 & 77 & 78 & 77 & 80 & 84 & 76 & 74 & 73 & 77 \\
\hline 2015 & 68 & 71 & 75 & 74 & 78 & 74 & 75 & 80 & 73 & 76 & 76 & 78 & 75 \\
\hline 2016 & 67 & 75 & 75 & 75 & 74 & 81 & 81 & 78 & 79 & 80 & 81 & 80 & 77 \\
\hline 2017 & 77 & 77 & 80 & 77 & 77 & 79 & 80 & 82 & 79 & 83 & 82 & 78 & 79 \\
\hline 2018 & 81 & 82 & 84 & 73 & 77 & 82 & 69 & 76 & & & & & \\
\hline
\end{tabular}




\begin{tabular}{|c|c|c|c|c|c|c|c|c|c|c|c|c|c|}
\hline Year & Jan. & Feb. & Mar. & Apr. & May & June & July & Aug. & Sept & Oct. & Nov. & Dec. & Annual \\
\hline \multicolumn{14}{|c|}{$\begin{array}{l}\text { 15. Labour utilization rate, industry (normal monthly level }=100 \text { ) } \\
\text { 15. Загрузка наличной рабочей силы, промышленность } \\
\text { (нормальный месячный уровень }=100 \text { ) }\end{array}$} \\
\hline 1994 & 75 & 76 & 73 & 74 & 72 & 69 & 73 & 75 & 76 & 78 & 78 & 79 & 75 \\
\hline 1995 & 78 & 76 & 81 & 78 & 75 & 78 & 78 & 79 & 78 & 78 & 77 & 73 & 77 \\
\hline 1996 & 73 & 73 & 73 & 75 & 72 & 75 & 76 & 69 & 72 & 70 & 71 & 72 & 73 \\
\hline 1997 & 69 & 72 & 73 & 74 & 75 & 74 & 74 & 76 & 78 & 77 & 76 & 78 & 75 \\
\hline 1998 & 74 & 75 & 78 & 77 & 71 & 74 & 72 & 74 & 73 & 73 & 76 & 78 & 75 \\
\hline 1999 & 75 & 79 & 82 & 83 & 82 & 84 & 83 & 87 & 85 & 85 & 87 & 85 & 83 \\
\hline 2000 & 84 & 85 & 85 & 86 & 86 & 89 & 88 & 89 & 89 & 86 & 88 & 88 & 87 \\
\hline 2001 & 84 & 86 & 87 & 86 & 87 & 88 & 86 & 88 & 88 & 87 & 88 & 85 & 87 \\
\hline 2002 & 85 & 83 & 88 & 86 & 85 & 85 & 85 & 89 & 89 & 90 & 89 & 88 & 87 \\
\hline 2003 & 84 & 89 & 87 & 89 & 88 & 88 & 84 & 89 & 90 & 91 & 91 & 89 & 88 \\
\hline 2004 & 86 & 86 & 87 & 87 & 87 & 87 & 89 & 90 & 87 & 92 & 88 & 90 & 88 \\
\hline 2005 & 84 & 85 & 90 & 87 & 89 & 92 & 92 & 91 & 91 & 90 & 90 & 91 & 89 \\
\hline 2006 & 84 & 89 & 92 & 90 & 93 & 90 & 93 & 94 & 94 & 94 & 93 & 94 & 92 \\
\hline 2007 & 90 & 93 & 91 & 95 & 94 & 93 & 93 & 95 & 93 & 95 & 95 & 95 & 94 \\
\hline 2008 & 92 & 92 & 94 & 94 & 92 & 91 & 91 & 90 & 91 & 90 & 84 & 80 & 90 \\
\hline 2009 & 73 & 77 & 76 & 76 & 76 & 77 & 78 & 81 & 82 & 80 & 84 & 83 & 79 \\
\hline 2010 & 81 & 85 & 82 & 84 & 85 & 89 & 88 & 88 & 91 & 92 & 89 & 91 & 87 \\
\hline 2011 & 87 & 89 & 91 & 90 & 89 & 87 & 89 & 91 & 89 & 91 & 93 & 90 & 90 \\
\hline 2012 & 88 & 90 & 92 & 92 & 93 & 89 & 93 & 93 & 91 & 91 & 91 & 89 & 91 \\
\hline 2013 & 87 & 91 & 91 & 89 & 88 & 89 & 88 & 87 & 88 & 87 & 90 & 84 & 88 \\
\hline 2014 & 89 & 86 & 88 & 87 & 85 & 88 & 85 & 90 & 92 & 88 & 83 & 86 & 87 \\
\hline 2015 & 78 & 83 & 84 & 85 & 88 & 87 & 87 & 87 & 89 & 86 & 88 & 90 & 86 \\
\hline 2016 & 80 & 88 & 83 & 87 & 87 & 91 & 87 & 88 & 89 & 90 & 92 & 86 & 87 \\
\hline 2017 & 87 & 88 & 91 & 92 & 91 & 92 & 90 & 92 & 91 & 94 & 91 & 92 & 91 \\
\hline 2018 & 89 & 94 & 90 & 84 & 87 & 91 & 86 & 86 & & & & & \\
\hline \multicolumn{14}{|c|}{ 16. Stocks of finished products, industry (normal monthly level $=100$ ) } \\
\hline 1993 & 83 & 92 & 82 & 95 & 91 & 91 & 87 & 95 & 83 & 97 & 109 & 104 & 92 \\
\hline 1994 & 101 & 103 & 104 & 116 & 107 & 96 & 93 & 84 & 75 & 89 & 84 & 78 & 94 \\
\hline 1995 & 81 & 74 & 82 & 90 & 78 & 77 & 74 & 78 & 78 & 89 & 88 & 96 & 82 \\
\hline 1996 & 95 & 101 & 93 & 103 & 97 & 102 & 94 & 91 & 86 & 90 & 84 & 88 & 94 \\
\hline 1997 & 90 & 88 & 99 & 95 & 92 & 90 & 83 & 82 & 81 & 79 & 86 & 82 & 87 \\
\hline 1998 & 83 & 91 & 90 & 92 & 90 & 91 & 89 & 78 & 80 & 80 & 79 & 78 & 85 \\
\hline 1999 & 76 & 79 & 83 & 79 & 77 & 75 & 74 & 75 & 70 & 75 & 75 & 73 & 76 \\
\hline 2000 & 74 & 78 & 78 & 84 & 86 & 82 & 82 & 80 & 84 & 78 & 80 & 74 & 80 \\
\hline 2001 & 85 & 83 & 84 & 86 & 89 & 88 & 84 & 85 & 83 & 82 & 84 & 83 & 85 \\
\hline 2002 & 91 & 94 & 94 & 93 & 93 & 88 & 92 & 90 & 87 & 89 & 86 & 84 & 90 \\
\hline 2003 & 86 & 88 & 86 & 94 & 86 & 89 & 88 & 89 & 85 & 91 & 88 & 84 & 88 \\
\hline 2004 & 90 & 91 & 90 & 93 & 89 & 87 & 80 & 85 & 81 & 91 & 95 & 92 & 89 \\
\hline 2005 & 91 & 99 & 94 & 88 & 94 & 88 & 89 & 87 & 86 & 91 & 87 & 84 & 90 \\
\hline 2006 & 80 & 102 & 95 & 91 & 91 & 89 & 89 & 84 & 86 & 81 & 90 & 84 & 88 \\
\hline 2007 & 82 & 90 & 88 & 89 & 87 & 91 & 82 & 85 & 87 & 86 & 84 & 88 & 87 \\
\hline 2008 & 85 & 88 & 89 & 88 & 90 & 93 & 92 & 94 & 97 & 91 & 99 & 87 & 91 \\
\hline 2009 & 102 & 90 & 98 & 97 & 98 & 92 & 95 & 92 & 96 & 88 & 92 & 88 & 94 \\
\hline 2010 & 92 & 90 & 97 & 90 & 89 & 88 & 94 & 85 & 86 & 89 & 88 & 89 & 90 \\
\hline 2011 & 94 & 89 & 96 & 95 & 91 & 98 & 84 & 92 & 85 & 88 & 85 & 88 & 90 \\
\hline 2012 & 90 & 93 & 89 & 92 & 93 & 95 & 100 & 84 & 91 & 83 & 93 & 97 & 92 \\
\hline 2013 & 99 & 102 & 100 & 92 & 95 & 92 & 94 & 94 & 84 & 85 & 89 & 97 & 94 \\
\hline 2014 & 93 & 95 & 96 & 94 & 89 & 92 & 87 & 92 & 99 & 95 & 92 & 96 & 93 \\
\hline 2015 & 91 & 84 & 99 & 89 & 92 & 93 & 87 & 90 & 86 & 88 & 94 & 83 & 90 \\
\hline 2016 & 83 & 76 & 92 & 87 & 97 & 80 & 90 & 89 & 84 & 87 & 85 & 85 & 86 \\
\hline 2017 & 83 & 89 & 84 & 74 & 86 & 84 & 93 & 79 & 82 & 85 & 97 & 84 & 85 \\
\hline 2018 & 76 & 95 & 97 & 82 & 87 & 89 & 79 & 80 & & & & & \\
\hline
\end{tabular}




\begin{tabular}{|c|c|c|c|c|c|c|c|c|c|c|c|c|c|}
\hline Year & Jan. & Feb. & Mar. & Apr. & May & June & July & Aug. & Sept & Oct. & Nov. & Dec. & Annual \\
\hline \multicolumn{14}{|c|}{$\begin{array}{l}\text { 17. Order-book level, industry (normal monthly level }=100 \text { ) } \\
\text { ель заказов, промышленность (нормальный месячный уровень = 100) }\end{array}$} \\
\hline 1993 & 82 & 91 & 83 & 82 & 86 & 81 & 83 & 78 & 79 & 76 & 72 & 74 & 81 \\
\hline 1994 & 71 & 70 & 65 & 66 & 66 & 65 & 65 & 72 & 73 & 72 & 75 & 74 & 70 \\
\hline 1995 & 74 & 70 & 74 & 73 & 70 & 73 & 74 & 68 & 71 & 68 & 67 & 66 & 71 \\
\hline 1996 & 62 & 61 & 61 & 66 & 64 & 70 & 63 & 59 & 63 & 59 & 59 & 61 & 62 \\
\hline 1997 & 58 & 56 & 59 & 59 & 63 & 64 & 63 & 66 & 67 & 67 & 69 & 68 & 63 \\
\hline 1998 & 67 & 66 & 65 & 67 & 60 & 61 & 65 & 62 & 66 & 66 & 70 & 70 & 65 \\
\hline 1999 & 70 & 77 & 74 & 78 & 75 & 81 & 82 & 81 & 78 & 83 & 76 & 77 & 78 \\
\hline 2000 & 78 & 77 & 80 & 79 & 80 & 81 & 83 & 84 & 80 & 80 & 79 & 76 & 80 \\
\hline 2001 & 77 & 76 & 79 & 78 & 76 & 77 & 81 & 82 & 81 & 81 & 79 & 76 & 79 \\
\hline 2002 & 74 & 77 & 75 & 76 & 77 & 79 & 77 & 81 & 83 & 83 & 78 & 82 & 79 \\
\hline 2003 & 75 & 81 & 76 & 78 & 76 & 81 & 79 & 80 & 85 & 83 & 85 & 81 & 80 \\
\hline 2004 & 82 & 82 & 82 & 83 & 83 & 81 & 80 & 84 & 83 & 83 & 81 & 81 & 82 \\
\hline 2005 & 74 & 78 & 83 & 80 & 82 & 83 & 85 & 85 & 85 & 85 & 83 & 84 & 82 \\
\hline 2006 & 79 & 81 & 85 & 86 & 89 & 85 & 91 & 91 & 91 & 90 & 90 & 92 & 87 \\
\hline 2007 & 84 & 86 & 89 & 91 & 92 & 93 & 93 & 92 & 91 & 91 & 88 & 89 & 90 \\
\hline 2008 & 86 & 88 & 90 & 89 & 86 & 87 & 87 & 86 & 86 & 79 & 70 & 68 & 84 \\
\hline 2009 & 63 & 65 & 65 & 66 & 65 & 68 & 71 & 72 & 76 & 72 & 74 & 77 & 70 \\
\hline 2010 & 77 & 76 & 75 & 78 & 79 & 81 & 79 & 80 & 81 & 86 & 80 & 84 & 80 \\
\hline 2011 & 79 & 85 & 85 & 84 & 86 & 82 & 87 & 88 & 87 & 85 & 85 & 84 & 85 \\
\hline 2012 & 82 & 79 & 86 & 86 & 89 & 84 & 84 & 87 & 85 & 86 & 84 & 83 & 85 \\
\hline 2013 & 80 & 87 & 84 & 84 & 82 & 84 & 82 & 82 & 84 & 82 & 87 & 80 & 83 \\
\hline 2014 & 84 & 85 & 84 & 80 & 79 & 83 & 80 & 86 & 88 & 84 & 79 & 80 & 83 \\
\hline 2015 & 74 & 77 & 78 & 82 & 83 & 79 & 77 & 82 & 81 & 80 & 82 & 82 & 80 \\
\hline 2016 & 76 & 79 & 79 & 80 & 90 & 90 & 83 & 83 & 83 & 84 & 87 & 82 & 83 \\
\hline 2017 & 84 & 86 & 87 & 82 & 84 & 88 & 85 & 84 & 87 & 89 & 86 & 85 & 86 \\
\hline 2018 & 85 & 87 & 86 & 80 & 88 & 91 & 75 & 74 & & & & & \\
\hline \multicolumn{14}{|c|}{$\begin{array}{l}\text { 19. Share of enterprises in 'good' or 'normal' financial conditions, industry (\%) } \\
\text { 19. Доля предприятий в "хорошем" или "нормальном" финансовом состоянии, промышленность (\%) }\end{array}$} \\
\hline 1993 & 42 & 41 & 46 & 44 & 43 & 41 & 42 & 31 & 37 & 28 & 19 & 21 & 36 \\
\hline 1994 & 21 & 20 & 18 & 19 & 19 & 23 & 18 & 25 & 24 & 27 & 33 & 35 & 24 \\
\hline 1995 & 27 & 25 & 36 & 29 & 28 & 29 & 32 & 26 & 28 & 23 & 17 & 19 & 27 \\
\hline 1996 & 12 & 16 & 15 & 14 & 14 & 13 & 19 & 15 & 19 & 15 & 17 & 17 & 16 \\
\hline 1997 & 18 & 18 & 17 & 17 & 18 & 24 & 20 & 24 & 31 & 32 & 25 & 27 & 23 \\
\hline 1998 & 24 & 19 & 21 & 17 & 16 & 18 & 20 & 13 & 16 & 22 & 26 & 32 & 20 \\
\hline 1999 & 30 & 41 & 41 & 41 & 45 & 48 & 47 & 49 & 54 & 52 & 48 & 47 & 45 \\
\hline 2000 & 49 & 53 & 51 & 50 & 56 & 54 & 54 & 58 & 61 & 56 & 51 & 52 & 54 \\
\hline 2001 & 47 & 49 & 49 & 51 & 52 & 60 & 61 & 58 & 59 & 59 & 55 & 54 & 54 \\
\hline 2002 & 47 & 39 & 46 & 43 & 47 & 52 & 51 & 55 & 49 & 54 & 45 & 55 & 49 \\
\hline 2003 & 50 & 47 & 55 & 57 & 53 & 60 & 57 & 62 & 65 & 69 & 59 & 60 & 58 \\
\hline 2004 & 51 & 55 & 61 & 64 & 63 & 65 & 65 & 68 & 65 & 68 & 65 & 62 & 63 \\
\hline 2005 & 56 & 54 & 69 & 67 & 69 & 71 & 78 & 74 & 73 & 73 & 71 & 75 & 69 \\
\hline 2006 & 59 & 62 & 71 & 69 & 69 & 75 & 79 & 83 & 81 & 79 & 79 & 83 & 74 \\
\hline 2007 & 72 & 74 & 76 & 75 & 77 & 83 & 78 & 82 & 76 & 81 & 82 & 80 & 78 \\
\hline 2008 & 72 & 73 & 75 & 73 & 74 & 72 & 72 & 72 & 69 & 58 & 51 & 48 & 67 \\
\hline 2009 & 36 & 42 & 42 & 41 & 43 & 52 & 53 & 56 & 58 & 55 & 64 & 63 & 50 \\
\hline 2010 & 53 & 54 & 55 & 61 & 61 & 69 & 67 & 69 & 74 & 74 & 67 & 69 & 64 \\
\hline 2011 & 63 & 56 & 62 & 71 & 71 & 67 & 76 & 73 & 77 & 81 & 81 & 73 & 71 \\
\hline 2012 & 66 & 70 & 72 & 76 & 78 & 63 & 82 & 80 & 86 & 84 & 73 & 77 & 76 \\
\hline 2013 & 72 & 71 & 80 & 63 & 71 & 68 & 66 & 72 & 75 & 80 & 76 & 73 & 72 \\
\hline 2014 & 63 & 61 & 65 & 64 & 67 & 66 & 71 & 71 & 75 & 74 & 75 & 67 & 68 \\
\hline 2015 & 55 & 61 & 71 & 74 & 67 & 69 & 64 & 71 & 67 & 68 & 69 & 78 & 68 \\
\hline 2016 & 70 & 69 & 66 & 69 & 71 & 83 & 71 & 69 & 77 & 76 & 80 & 72 & 73 \\
\hline 2017 & 76 & 64 & 74 & 66 & 82 & 77 & 79 & 76 & 72 & 84 & 80 & 79 & 76 \\
\hline 2018 & 80 & 76 & 86 & 72 & 82 & 87 & 66 & 74 & & & & & \\
\hline
\end{tabular}


The Russian Economic Barometer, Vol. XXVII, 3/4, 2018

\begin{tabular}{|c|c|c|c|c|c|c|c|c|c|c|c|c|c|}
\hline Year & Jan. & Feb. & Mar. & Apr. & May & June & July & Aug. & Sept & Oct. & Nov. & Dec. & Annual \\
\hline \multicolumn{14}{|c|}{$\begin{array}{l}\text { 20. Share of enterprises not buying equipment for } 2 \text { and more months, industry (\%) } \\
\text { 20. Доля предприятий, не закупающих оборудование } 2 \text { и более месяцев подряд, промышленность (\% }\end{array}$} \\
\hline 1993 & 50 & 49 & 47 & 62 & 48 & 57 & 50 & 57 & 53 & 58 & 56 & 54 & 53 \\
\hline 1994 & 53 & 65 & 67 & 69 & 65 & 65 & 60 & 58 & 64 & 65 & 60 & 55 & 62 \\
\hline 1995 & 61 & 65 & 57 & 59 & 61 & 50 & 55 & 60 & 58 & 54 & 61 & 58 & 58 \\
\hline 1996 & 64 & 62 & 65 & 67 & 63 & 69 & 65 & 68 & 66 & 67 & 66 & 66 & 66 \\
\hline 1997 & 70 & 68 & 72 & 74 & 63 & 63 & 70 & 67 & 57 & 56 & 61 & 63 & 65 \\
\hline 1998 & 60 & 65 & 64 & 66 & 69 & 66 & 62 & 71 & 72 & 66 & 67 & 65 & 66 \\
\hline 1999 & 62 & 69 & 64 & 58 & 55 & 55 & 55 & 54 & 53 & 57 & 55 & 54 & 58 \\
\hline 2000 & 54 & 52 & 52 & 54 & 48 & 51 & 48 & 49 & 49 & 48 & 49 & 51 & 50 \\
\hline 2001 & 50 & 51 & 51 & 50 & 48 & 43 & 44 & 46 & 41 & 41 & 39 & 41 & 45 \\
\hline 2002 & 43 & 46 & 45 & 47 & 45 & 44 & 44 & 40 & 38 & 45 & 48 & 49 & 45 \\
\hline 2003 & 44 & 43 & 45 & 47 & 42 & 39 & 38 & 41 & 45 & 40 & 43 & 36 & 42 \\
\hline 2004 & 41 & 41 & 45 & 42 & 39 & 45 & 40 & 37 & 44 & 43 & 40 & 40 & 41 \\
\hline 2005 & 43 & 46 & 39 & 41 & 36 & 42 & 38 & 34 & 38 & 40 & 43 & 43 & 40 \\
\hline 2006 & 46 & 36 & 36 & 41 & 33 & 33 & 25 & 33 & 27 & 29 & 32 & 31 & 34 \\
\hline 2007 & 35 & 30 & 30 & 25 & 31 & 27 & 26 & 30 & 33 & 29 & 32 & 36 & 30 \\
\hline 2008 & 35 & 35 & 30 & 31 & 26 & 29 & 27 & 28 & 37 & 3 & 38 & 44 & 33 \\
\hline 2009 & 54 & 51 & 53 & 50 & 50 & 52 & 53 & 39 & 43 & 47 & 39 & 43 & 48 \\
\hline 2010 & 39 & 48 & 51 & 45 & 47 & 45 & 47 & 47 & 47 & 44 & 44 & 41 & 45 \\
\hline 2011 & 34 & 44 & 42 & 39 & 39 & 36 & 38 & 4 & 37 & 35 & 38 & 41 & 40 \\
\hline 2012 & 37 & 45 & 39 & 28 & 42 & 41 & 30 & 31 & 32 & 37 & 36 & 33 & 36 \\
\hline 2013 & 38 & 36 & 31 & 32 & 34 & 28 & 40 & 33 & 34 & 31 & 35 & 34 & 34 \\
\hline 2014 & 38 & 37 & 34 & 43 & 43 & 39 & 3 & $\checkmark$ & 35 & 40 & 45 & 41 & 39 \\
\hline 2015 & 47 & 40 & 33 & 41 & 37 & 38 & 40 & 39 & 48 & 44 & 44 & 41 & 41 \\
\hline 2016 & 46 & 44 & 51 & 46 & 45 & 40 & 38 & 46 & 51 & 43 & 45 & 50 & 45 \\
\hline 2017 & 50 & 45 & 38 & 46 & 41 & 40 & 37 & 28 & 38 & 38 & 37 & 37 & 40 \\
\hline 2018 & 42 & 29 & 32 & 36 & 36 & 28 & 44 & 47 & & & & & \\
\hline \multicolumn{14}{|c|}{$\begin{array}{l}\text { 31. Anticipated interest rates on bank credits (in roubles) to be received in the course of } 3 \text { months, industry } \\
\text { ( } \% \text { on annual basis) }\end{array}$} \\
\hline \multicolumn{14}{|c|}{$\begin{array}{l}\text { 31. Процентные ставки по банковским кредитам (в рублях), привлекаемым в ближайшие три месяца, } \\
\text { промышленность (\% в годовом исчислении) }\end{array}$} \\
\hline 1994 & 199 & 201 & 222 & 199 & 206 & 165 & 158 & 133 & 128 & 140 & 144 & 164 & 172 \\
\hline 1995 & 163 & 166 & 163 & 170 & 156 & 152 & 130 & 128 & 140 & 136 & 144 & 130 & 148 \\
\hline 1996 & 124 & 121 & 111 & 109 & 112 & 101 & 106 & 89 & 82 & 76 & 64 & 58 & 96 \\
\hline 1997 & 48 & 47 & 43 & 40 & 34 & 29 & 28 & 2 & 23 & 2 & 2 & 3 & 33 \\
\hline 1998 & 26 & 26 & 23 & 23 & 31 & 28 & 27 & 35 & 32 & 37 & 38 & 30 & 30 \\
\hline 1999 & 34 & 36 & 29 & 31 & 37 & 37 & 32 & 31 & 33 & 32 & 34 & 28 & 33 \\
\hline 2000 & 29 & 31 & 26 & 26 & 24 & 25 & 2 & 2 & 20 & 2 & $2 C$ & 19 & 24 \\
\hline 2001 & 21 & 19 & 20 & 20 & 20 & 20 & 20 & 20 & 19 & 20 & 18 & 19 & 20 \\
\hline 2002 & 20 & 20 & 19 & 19 & 20 & 19 & 19 & 19 & 19 & 18 & 19 & 18 & 19 \\
\hline 2003 & 18 & 18 & 1 & 17 & 17 & 16 & 15 & 16 & 15 & 1 & 14 & 14 & 16 \\
\hline 2004 & 15 & 15 & 16 & 15 & 14 & 14 & 14 & 14 & 14 & 13 & 14 & 14 & 14 \\
\hline 2005 & 14 & 14 & 14 & 14 & 13 & 14 & 13 & 12 & 13 & 13 & 13 & 13 & 13 \\
\hline & 1 & 1 & 1. & 1 & 12 & 11 & 1 & 1 & 11 & I & 1 & & 12 \\
\hline 2007 & 11 & 11 & 11 & 11 & 11 & 12 & 11 & 11 & 11 & 10 & 11 & 11 & 11 \\
\hline 2008 & 11 & 11 & 11 & 11 & 12 & 11 & 11 & 12 & 13 & 15 & 14 & 15 & 12 \\
\hline 2009 & 15 & 15 & 15 & 16 & 14 & 15 & 1 & 1 & 15 & 1 & 1 & 1 & 15 \\
\hline 2010 & 14 & 13 & 14 & 14 & 13 & 13 & 12 & 12 & 13 & 12 & 12 & 12 & 13 \\
\hline 2011 & 12 & 12 & 11 & 11 & 12 & 11 & 11 & 11 & 11 & 1 & 11 & 12 & 11 \\
\hline 2012 & 11 & 11 & 11 & 11 & 12 & 11 & 11 & 11 & 12 & 1 & 12 & 11 & 11 \\
\hline 2013 & 12 & 12 & 12 & 11 & 11 & 11 & 12 & 12 & 11 & 11 & 12 & 11 & 11 \\
\hline 2014 & 11 & 12 & 11 & 12 & 12 & 12 & 12 & 12 & 12 & 12 & 12 & 14 & 12 \\
\hline 2015 & 16 & 16 & 16 & 14 & 15 & 15 & 15 & 15 & 14 & 13 & 14 & 14 & 15 \\
\hline 2016 & 14 & 15 & 14 & 15 & 13 & 13 & 14 & 14 & 12 & 12 & 12 & 13 & 13 \\
\hline 2017 & 11 & 12 & 12 & 11 & 11 & 11 & 11 & 10 & 9 & 10 & 12 & 10 & 11 \\
\hline 2018 & 10 & 10 & 11 & 11 & 10 & 9 & 10 & 9 & & & & & \\
\hline
\end{tabular}




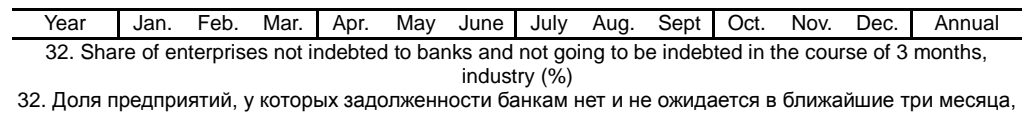

32. Доля предприятий, у которых задолженности банкам нет и не ожидается в ближайшие три месяца,
промышленность (\%)

\begin{tabular}{|c|c|c|c|c|c|c|c|c|c|c|c|c|c|}
\hline 1994 & 21 & 23 & 20 & 19 & 24 & 17 & 24 & 21 & 26 & 21 & 21 & 31 & 22 \\
\hline 1995 & 25 & 27 & 23 & 31 & 32 & 28 & 35 & 23 & 27 & 30 & 30 & 26 & 28 \\
\hline 1996 & 31 & 32 & 27 & 34 & 33 & 32 & 33 & 28 & 34 & 30 & 32 & 42 & 32 \\
\hline 1997 & 34 & 36 & 36 & 33 & 42 & 40 & 41 & 37 & 38 & 40 & 40 & 38 & 38 \\
\hline 1998 & 43 & 43 & 43 & 49 & 44 & 46 & 42 & 49 & 54 & 49 & 55 & 55 & 48 \\
\hline 1999 & 54 & 55 & 50 & 53 & 54 & 45 & 47 & 53 & 54 & 50 & 48 & 52 & 51 \\
\hline 2000 & 47 & 48 & 45 & 47 & 49 & 52 & 45 & 47 & 55 & 51 & 46 & 48 & 48 \\
\hline 2001 & 45 & 44 & 39 & 40 & 42 & 39 & 41 & 41 & 37 & 40 & 35 & 41 & 40 \\
\hline 2002 & 36 & 32 & 35 & 37 & 35 & 42 & 32 & 39 & 38 & 40 & 38 & 39 & 37 \\
\hline 2003 & 39 & 35 & 34 & 32 & 31 & 35 & 29 & 34 & 37 & 35 & 29 & 37 & 34 \\
\hline 2004 & 32 & 28 & 33 & 33 & 29 & 34 & 34 & 32 & 30 & 24 & 36 & 34 & 32 \\
\hline 2005 & 32 & 32 & 26 & 23 & 31 & 32 & 34 & 28 & 29 & 31 & 29 & 31 & 30 \\
\hline 2006 & 33 & 35 & 29 & 32 & 30 & 28 & 32 & 25 & 26 & 31 & 33 & 30 & 30 \\
\hline 2007 & 33 & 31 & 33 & 29 & 26 & 30 & 34 & 34 & 32 & 25 & 38 & 35 & 32 \\
\hline 2008 & 33 & 31 & 32 & 38 & 35 & 34 & 30 & 33 & 33 & 28 & 39 & 34 & 33 \\
\hline 2009 & 34 & 37 & 37 & 39 & 39 & 38 & 43 & 34 & 44 & 42 & 44 & 38 & 39 \\
\hline 2010 & 42 & 43 & 39 & 39 & 34 & 36 & 43 & 44 & 53 & 45 & 36 & 36 & 41 \\
\hline 2011 & 38 & 41 & 37 & 40 & 37 & 40 & 41 & 40 & 42 & 43 & 41 & 43 & 40 \\
\hline 2012 & 43 & 49 & 49 & 42 & 39 & 45 & 46 & 40 & 46 & 47 & 41 & 39 & 44 \\
\hline 2013 & 36 & 40 & 43 & 43 & 36 & 44 & 45 & 37 & 38 & 37 & 40 & 39 & 40 \\
\hline 2014 & 33 & 36 & 50 & 35 & 36 & 37 & 44 & 45 & 44 & 44 & 47 & 41 & 41 \\
\hline 2015 & 47 & 42 & 45 & 44 & 41 & 38 & 51 & 47 & 41 & 52 & 47 & 41 & 45 \\
\hline 2016 & 34 & 43 & 52 & 38 & 36 & 35 & 38 & 41 & 42 & 44 & 44 & 47 & 41 \\
\hline 2017 & 63 & 42 & 49 & 55 & 45 & 46 & 50 & 39 & 44 & 49 & 41 & 51 & 48 \\
\hline 2018 & 49 & 40 & 48 & 33 & 39 & 33 & 39 & 36 & & & & & \\
\hline \multicolumn{14}{|c|}{$\begin{array}{l}\text { 33. Indebtedness to banks, industry (normal monthly level = 100) } \\
\text { 33. Задолженность банкам, промышленность (нормальный месячный уровень = 100) }\end{array}$} \\
\hline 1996 & 107 & 109 & 104 & 117 & 127 & 115 & 108 & 124 & 111 & 130 & 133 & 130 & 118 \\
\hline 1997 & 128 & 120 & 112 & 112 & 107 & 114 & 112 & 115 & 114 & 95 & 92 & 95 & 110 \\
\hline 1998 & 99 & 109 & 99 & 91 & 83 & 109 & 118 & 112 & 108 & 95 & 103 & 101 & 102 \\
\hline 1999 & 80 & 99 & 97 & 95 & 90 & 84 & 84 & 85 & 65 & 85 & 77 & 81 & 85 \\
\hline 2000 & 75 & 80 & 76 & 72 & 68 & 80 & 78 & 82 & 85 & 66 & 91 & 74 & 77 \\
\hline 2001 & 82 & 70 & 79 & 79 & 77 & 79 & 69 & 89 & 83 & 78 & 67 & 85 & 78 \\
\hline 2002 & 88 & 81 & 83 & 88 & 88 & 84 & 80 & 80 & 88 & 92 & 86 & 88 & 86 \\
\hline 2003 & 94 & 86 & 95 & 93 & 87 & 99 & 88 & 93 & 89 & 92 & 85 & 90 & 91 \\
\hline 2004 & 91 & 91 & 87 & 91 & 84 & 83 & 82 & 80 & 91 & 85 & 81 & 82 & 86 \\
\hline 2005 & 85 & 95 & 99 & 81 & 76 & 79 & 81 & 86 & 87 & 77 & 85 & 80 & 84 \\
\hline 2006 & 84 & 95 & 82 & 86 & 85 & 71 & 83 & 80 & 77 & 76 & 82 & 79 & 82 \\
\hline 2007 & 80 & 84 & 83 & 78 & 85 & 81 & 73 & 74 & 78 & 78 & 79 & 85 & 80 \\
\hline 2008 & 86 & 81 & 85 & 79 & 82 & 87 & 79 & 85 & 91 & 89 & 99 & 94 & 86 \\
\hline 2009 & 101 & 95 & 94 & 98 & 101 & 93 & 103 & 90 & 103 & 85 & 101 & 95 & 97 \\
\hline 2010 & 98 & 104 & 117 & 94 & 95 & 94 & 90 & 83 & 94 & 90 & 97 & 91 & 96 \\
\hline 2011 & 100 & 96 & 97 & 94 & 96 & 93 & 97 & 89 & 98 & 88 & 75 & 78 & 92 \\
\hline 2012 & 79 & 87 & 106 & 88 & 90 & 79 & 82 & 85 & 69 & 73 & 93 & 80 & 84 \\
\hline 2013 & 82 & 86 & 77 & 85 & 84 & 65 & 69 & 79 & 77 & 81 & 72 & 83 & 78 \\
\hline 2014 & 89 & 86 & 85 & 81 & 78 & 72 & 84 & 85 & 80 & 72 & 76 & 75 & 80 \\
\hline 2015 & 70 & 79 & 72 & 87 & 85 & 82 & 78 & 72 & 80 & 88 & 91 & 80 & 80 \\
\hline 2016 & 71 & 76 & 70 & 76 & 68 & 67 & 88 & 82 & 89 & 66 & 88 & 87 & 77 \\
\hline 2017 & 69 & 86 & 70 & 84 & 67 & 85 & 78 & 76 & 104 & 100 & 95 & 78 & 83 \\
\hline 2018 & 78 & 71 & 71 & 89 & 76 & 85 & 84 & 76 & & & & & \\
\hline
\end{tabular}


The Russian Economic Barometer, Vol. XXVII, 3/4, 2018

\begin{tabular}{|c|c|c|c|c|c|c|c|c|c|c|c|c|c|}
\hline Year & Jan. & Feb. & Mar. & Apr. & May & June & July & Aug. & Sept & Oct. & Nov. & Dec. & Annual \\
\hline \multicolumn{14}{|c|}{$\begin{array}{l}\text { 34. Share of enterprises not going to make new bank borrowings in the next } 3 \text { months, industry (\%) } \\
\text { 34. Доля предприятий, не собирающихся брать новые ссуды у банков в ближайшие } 3 \text { месяца, } \\
\text { промышленность (\%) }\end{array}$} \\
\hline 1994 & 31 & 33 & 34 & 30 & 39 & 33 & 37 & 38 & 38 & 38 & 36 & 48 & 36 \\
\hline 1995 & 49 & 45 & 44 & 44 & 51 & 44 & 52 & 42 & 52 & 48 & 51 & 42 & 47 \\
\hline 1996 & 50 & 43 & 42 & 48 & 48 & 43 & 48 & 48 & 53 & 49 & 53 & 60 & 49 \\
\hline 1997 & 49 & 56 & 51 & 56 & 57 & 58 & 55 & 53 & 55 & 53 & 58 & 56 & 55 \\
\hline 1998 & 58 & 61 & 59 & 63 & 62 & 68 & 62 & 66 & 74 & 71 & 75 & 74 & 66 \\
\hline 1999 & 76 & 74 & 67 & 67 & 68 & 60 & 62 & 67 & 66 & 63 & 59 & 63 & 66 \\
\hline 2000 & 56 & 62 & 54 & 55 & 58 & 61 & 56 & 55 & 63 & 59 & 56 & 56 & 58 \\
\hline 2001 & 53 & 54 & 48 & 50 & 53 & 48 & 49 & 49 & 42 & 49 & 41 & 49 & 49 \\
\hline 2002 & 42 & 42 & 43 & 48 & 43 & 51 & 43 & 49 & 46 & 52 & 50 & 47 & 46 \\
\hline 2003 & 49 & 40 & 42 & 45 & 41 & 42 & 41 & 44 & 44 & 46 & 39 & 46 & 43 \\
\hline 2004 & 39 & 43 & 38 & 40 & 41 & 38 & 41 & 43 & 43 & 35 & 46 & 42 & 41 \\
\hline 2005 & 41 & 39 & 33 & 31 & 38 & 38 & 38 & 38 & 38 & 38 & 36 & 40 & 37 \\
\hline 2006 & 39 & 45 & 34 & 41 & 35 & 36 & 36 & 35 & 30 & 38 & 38 & 36 & 37 \\
\hline 2007 & 39 & 36 & 40 & 34 & 34 & 37 & 38 & 40 & 36 & 31 & 43 & 39 & 37 \\
\hline 2008 & 41 & 36 & 38 & 44 & 44 & 41 & 35 & 38 & 42 & 33 & 46 & 45 & 40 \\
\hline 2009 & 44 & 46 & 49 & 47 & 47 & 53 & 51 & 44 & 55 & 56 & 55 & 49 & 50 \\
\hline 2010 & 51 & 46 & 46 & 47 & 45 & 47 & 55 & 51 & 57 & 49 & 47 & 45 & 49 \\
\hline 2011 & 45 & 47 & 46 & 49 & 48 & 49 & 48 & 51 & 48 & 50 & 46 & 52 & 48 \\
\hline 2012 & 46 & 57 & 53 & 49 & 42 & 50 & 51 & 48 & 55 & 51 & 50 & 52 & 50 \\
\hline 2013 & 49 & 46 & 48 & 48 & 41 & 51 & 51 & 46 & 43 & 41 & 48 & 45 & 46 \\
\hline 2014 & 38 & 42 & 50 & 42 & 42 & 40 & 47 & 49 & 48 & 46 & 53 & 52 & 46 \\
\hline 2015 & 47 & 46 & 47 & 47 & 48 & 45 & 55 & 49 & 46 & 54 & 51 & 43 & 48 \\
\hline 2016 & 40 & 49 & 54 & 44 & 41 & 41 & 45 & 49 & 49 & 46 & 51 & 51 & 47 \\
\hline 2017 & 65 & 49 & 49 & 57 & 55 & 52 & 53 & 39 & 56 & 59 & 46 & 55 & 53 \\
\hline 2018 & 59 & 51 & 55 & 44 & 58 & 45 & 45 & 42 & & & & & \\
\hline \multicolumn{14}{|c|}{$\begin{array}{l}\text { 36. Share of sales for cash (ready money) in total sales, industry (\%) } \\
\text { 36. Доля продаж за наличные в продажах, промышленность (\%) }\end{array}$} \\
\hline 1997 & - & - & - & 18 & - & 16 & 16 & 15 & 15 & 15 & 15 & 16 & - \\
\hline 1998 & 15 & 16 & 13 & 15 & 13 & 16 & 16 & 17 & 18 & 17 & 20 & 19 & 16 \\
\hline 1999 & 21 & 20 & 20 & 20 & 24 & 25 & 25 & 24 & 26 & 27 & 26 & 27 & 23 \\
\hline 2000 & 26 & 28 & 27 & 28 & 27 & 29 & 28 & 30 & 25 & 27 & 28 & 32 & 28 \\
\hline 2001 & 30 & 27 & 29 & 26 & 26 & 27 & 29 & 28 & 29 & 28 & 27 & 26 & 28 \\
\hline 2002 & 26 & 24 & 22 & 24 & 23 & 26 & 25 & 21 & 24 & 28 & 26 & 24 & 24 \\
\hline 2003 & 25 & 22 & 22 & 19 & 24 & 21 & 24 & 22 & 24 & 23 & 24 & 22 & 23 \\
\hline 2004 & 24 & 25 & 23 & 24 & 22 & 26 & 22 & 23 & 24 & 21 & 21 & 22 & 23 \\
\hline 2005 & 20 & 20 & 22 & 18 & 21 & 18 & 19 & 20 & 16 & 22 & 22 & 20 & 20 \\
\hline 2006 & 22 & 16 & 20 & 19 & 15 & 16 & 15 & 17 & 14 & 19 & 16 & 18 & 17 \\
\hline 2007 & 15 & 14 & 13 & 16 & 15 & 11 & 15 & 13 & 12 & 19 & 10 & 9 & 14 \\
\hline 2008 & 13 & 13 & 10 & 16 & 8 & 12 & 16 & 12 & 13 & 15 & 13 & 14 & 13 \\
\hline 2009 & 12 & 12 & 12 & 18 & 13 & 13 & 9 & 12 & 15 & 13 & 14 & 13 & 13 \\
\hline 2010 & 13 & 14 & 12 & 11 & 11 & 10 & 12 & 10 & 12 & 7 & 7 & 9 & 11 \\
\hline 2011 & 6 & 11 & 12 & 8 & 7 & 9 & 11 & 9 & 11 & 10 & 12 & 8 & 10 \\
\hline 2012 & 10 & 6 & 7 & 8 & 7 & 8 & 8 & 9 & 8 & 9 & 7 & 11 & 8 \\
\hline 2013 & 8 & 7 & 5 & 4 & 9 & 6 & 8 & 7 & 9 & 5 & 5 & 9 & 7 \\
\hline 2014 & 6 & 6 & 4 & 6 & 8 & 3 & 7 & 8 & 5 & 11 & 8 & 9 & 7 \\
\hline 2015 & 7 & 4 & 7 & 8 & 4 & 5 & 5 & 5 & 4 & 6 & 5 & 5 & 5 \\
\hline 2016 & 7 & 6 & 7 & $-{ }^{1}$ & - & - & - & - & - & - & - & - & - \\
\hline
\end{tabular}

${ }^{1}$ Since April 2016 this index is not measured. 


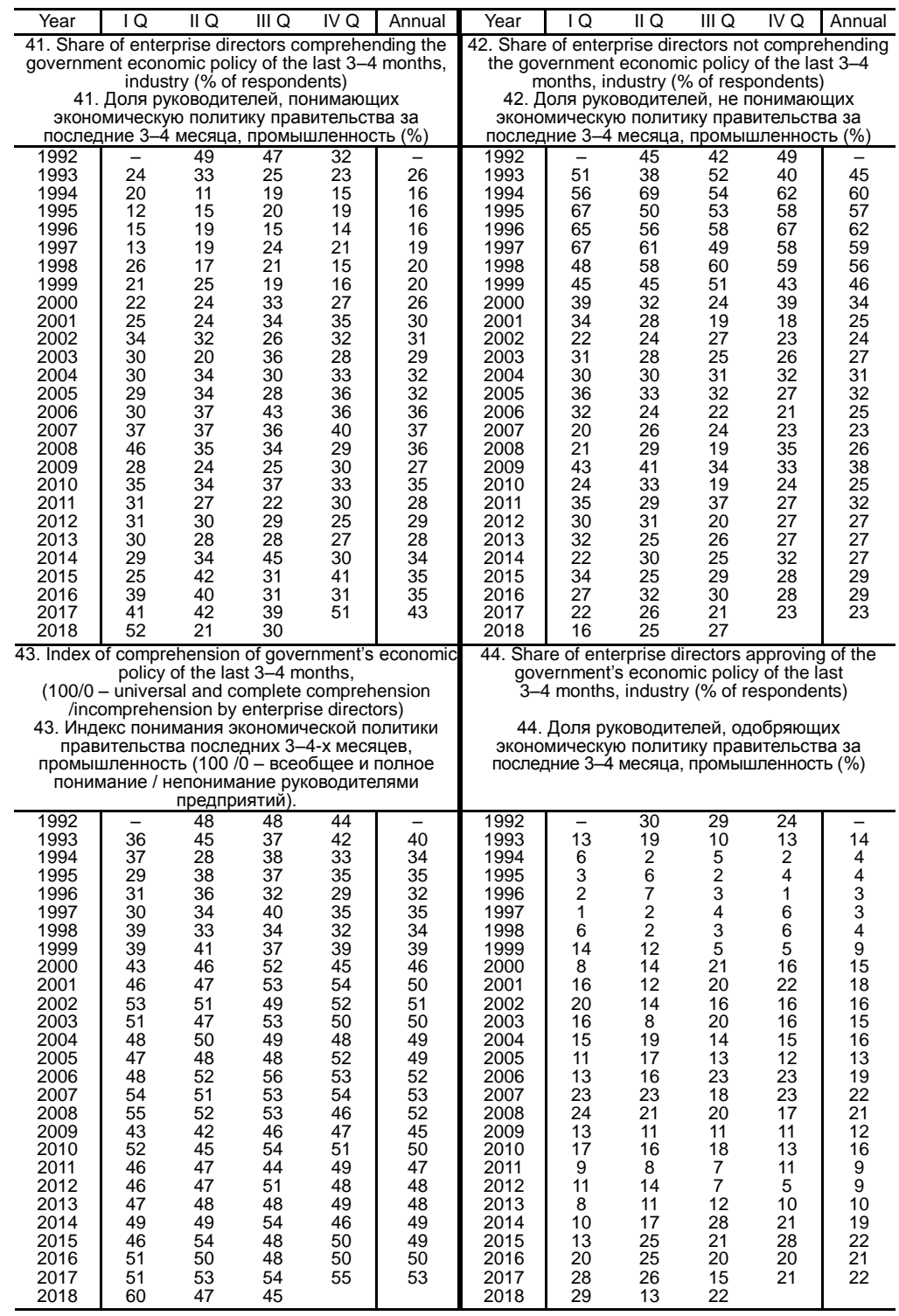


The Russian Economic Barometer, Vol. XXVII, 3/4, 2018

\begin{tabular}{|c|c|c|c|c|c|c|c|c|c|c|c|}
\hline Year & $I Q$ & $\mathrm{IIQ}$ & $\mathrm{IIIQ}$ & $I V Q$ & Annual & Year & $\overline{I Q}$ & $\| \mathrm{Q}$ & $\mathrm{IIIQ}$ & $I V Q$ & Annual \\
\hline \multicolumn{6}{|c|}{$\begin{array}{c}\text { 45. Доля руководителей, не одобряющих } \\
\text { экономическую политику правительства за последние } \\
\text { 3-4 месяца, промышленность (\%) }\end{array}$} & \multicolumn{6}{|c|}{$\begin{array}{c}\text { 46. Index of approval of government's economic } \\
\text { policy of the last 3-4 months, (100/0 - universal and } \\
\text { complete approval/disapproval by } \\
\text { enterprise directors) } \\
\text { 46. Индекс одобрения экономической политики } \\
\text { правительства последних } 3-4-x \text { месяцев, } \\
\text { промышленность (100/0- - всеобщее и полное } \\
\text { одобрение / неодобрение pуководителями } \\
\text { предприятий). }\end{array}$} \\
\hline $\begin{array}{l}1992 \\
1993 \\
1994 \\
1995 \\
1996 \\
1997 \\
1998 \\
1999 \\
2000 \\
2001 \\
2002 \\
2003 \\
2004 \\
2005 \\
2006 \\
2007 \\
2008 \\
2009 \\
2010 \\
2011 \\
2012 \\
2013 \\
2014 \\
2015 \\
2016 \\
2017 \\
2018\end{array}$ & $\begin{array}{l}- \\
48 \\
61 \\
68 \\
74 \\
75 \\
69 \\
45 \\
45 \\
33 \\
26 \\
38 \\
37 \\
45 \\
46 \\
29 \\
31 \\
39 \\
27 \\
41 \\
37 \\
37 \\
26 \\
32 \\
30 \\
19 \\
23\end{array}$ & $\begin{array}{l}39 \\
40 \\
61 \\
60 \\
63 \\
75 \\
68 \\
49 \\
30 \\
31 \\
32 \\
36 \\
35 \\
43 \\
39 \\
32 \\
26 \\
36 \\
37 \\
36 \\
27 \\
31 \\
24 \\
24 \\
30 \\
30 \\
18\end{array}$ & $\begin{array}{l}49 \\
55 \\
63 \\
63 \\
63 \\
62 \\
73 \\
61 \\
22 \\
23 \\
29 \\
34 \\
34 \\
41 \\
27 \\
31 \\
19 \\
36 \\
24 \\
38 \\
33 \\
31 \\
27 \\
23 \\
26 \\
21 \\
28\end{array}$ & $\begin{array}{l}44 \\
44 \\
73 \\
65 \\
75 \\
66 \\
64 \\
54 \\
38 \\
23 \\
29 \\
30 \\
44 \\
37 \\
25 \\
27 \\
31 \\
39 \\
32 \\
32 \\
29 \\
25 \\
33 \\
31 \\
24 \\
31\end{array}$ & $\begin{array}{l}- \\
47 \\
65 \\
64 \\
69 \\
69 \\
68 \\
52 \\
34 \\
28 \\
29 \\
34 \\
37 \\
41 \\
34 \\
30 \\
27 \\
37 \\
30 \\
37 \\
32 \\
31 \\
28 \\
28 \\
28 \\
25\end{array}$ & $\begin{array}{l}1992 \\
1993 \\
1994 \\
1995 \\
1996 \\
1997 \\
1998 \\
1999 \\
2000 \\
2001 \\
2002 \\
2003 \\
2004 \\
2005 \\
2006 \\
2007 \\
2008 \\
2009 \\
2010 \\
2011 \\
2012 \\
2013 \\
2014 \\
2015 \\
2016 \\
2017 \\
2018\end{array}$ & $\begin{array}{l}- \\
35 \\
28 \\
25 \\
23 \\
22 \\
26 \\
37 \\
37 \\
44 \\
47 \\
42 \\
41 \\
38 \\
39 \\
47 \\
47 \\
40 \\
44 \\
39 \\
40 \\
39 \\
43 \\
43 \\
44 \\
51{ }^{*} \\
51\end{array}$ & $\begin{array}{l}41 \\
39 \\
27 \\
30 \\
29 \\
23 \\
24 \\
36 \\
44 \\
42 \\
44 \\
35 \\
42 \\
39 \\
42 \\
46 \\
47 \\
40 \\
43 \\
40 \\
44 \\
42 \\
46 \\
48 \\
46 \\
45 \\
47\end{array}$ & $\begin{array}{l}40 \\
31 \\
27 \\
28 \\
26 \\
28 \\
23 \\
29 \\
48 \\
48 \\
44 \\
44 \\
43 \\
39 \\
47 \\
46 \\
40 \\
42 \\
48 \\
38 \\
41 \\
43 \\
48 \\
48 \\
46 \\
46 \\
44\end{array}$ & $\begin{array}{l}41 \\
37 \\
23 \\
27 \\
22 \\
26 \\
27 \\
32 \\
42 \\
48 \\
44 \\
44 \\
39 \\
41 \\
49 \\
48 \\
44 \\
39 \\
43 \\
43 \\
41 \\
44 \\
45 \\
46 \\
48 \\
44\end{array}$ & $\begin{array}{l}- \\
36 \\
26 \\
28 \\
25 \\
25 \\
25 \\
34 \\
43 \\
46 \\
45 \\
41 \\
41 \\
39 \\
44 \\
47 \\
45 \\
40 \\
44 \\
40 \\
41 \\
42 \\
45 \\
46 \\
46 \\
46\end{array}$ \\
\hline \multicolumn{6}{|c|}{$\begin{array}{l}\text { 47. Time remaining until the end of crisis, managers' } \\
\text { estimates, industry (years) }\end{array}$} & \multicolumn{6}{|c|}{$\begin{array}{l}\text { 48. Limitations to production: insufficient demand, } \\
\text { present situation, industry } \\
\text { (share of enterprises, \%) } \\
\text { 48. Ограничения производства: недостаток } \\
\text { спроса, текущая ситуация, промышленность (доля } \\
\text { предприятий, \%) }\end{array}$} \\
\hline $\begin{array}{l}1991 \\
1992 \\
1993 \\
1994 \\
1995 \\
1996 \\
1997 \\
1998 \\
1999 \\
2000 \\
2001 \\
2002 \\
2003 \\
2004 \\
2005 \\
2006 \\
2007 \\
2008 \\
2009 \\
2010 \\
2011 \\
2012 \\
2013 \\
2014 \\
2015 \\
2016 \\
2017 \\
2018\end{array}$ & $\begin{array}{c}- \\
- \\
5.8 \\
5.9 \\
8.2 \\
5.9 \\
7.5 \\
7.3 \\
7.3 \\
6.3 \\
7.0 \\
6.5 \\
7.5 \\
8.0 \\
8.0 \\
8.1 \\
6.8 \\
5.6 \\
3.7 \\
5.4 \\
4.0 \\
5.2 \\
5.0 \\
5.7 \\
5.2 \\
6.2 \\
6.5 \\
5.8\end{array}$ & $\begin{array}{l}- \\
6.2 \\
5.4 \\
6.5 \\
6.6 \\
6.3 \\
8.2 \\
7.1 \\
7.9 \\
5.3 \\
6.5 \\
7.8 \\
8.0 \\
8.3 \\
8.3 \\
7.5 \\
7.0 \\
6.4 \\
4.4 \\
4.3 \\
4.8 \\
5.7 \\
6.4 \\
6.0 \\
3.9 \\
6.5 \\
7.0 \\
7.7\end{array}$ & $\begin{array}{l}- \\
5.8 \\
6.5 \\
6.1 \\
6.6 \\
7.2 \\
7.8 \\
8.3 \\
8.0 \\
5.9 \\
6.2 \\
7.0 \\
7.1 \\
7.0 \\
7.5 \\
6.3 \\
6.7 \\
5.7 \\
4.3 \\
3.8 \\
5.2 \\
5.9 \\
5.9 \\
6.3 \\
5.0 \\
5.6 \\
5.7 \\
8.4\end{array}$ & $\begin{array}{l}-.9 \\
5.1 \\
6.8 \\
6.8 \\
7.5 \\
7.6 \\
6.7 \\
7.6 \\
6.7 \\
6.0 \\
7.2 \\
7.4 \\
7.2 \\
7.6 \\
6.5 \\
6.8 \\
5.7 \\
4.3 \\
4.1 \\
5.7 \\
6.0 \\
5.6 \\
5.6 \\
5.8 \\
5.8 \\
6.7\end{array}$ & $\begin{array}{l}- \\
- \\
6.0 \\
6.3 \\
7.1 \\
6.7 \\
7.8 \\
7.4 \\
7.7 \\
6.0 \\
6.4 \\
7.1 \\
7.5 \\
7.6 \\
7.8 \\
7.1 \\
6.8 \\
5.9 \\
4.2 \\
4.4 \\
4.9 \\
5.7 \\
5.7 \\
5.9 \\
5.0 \\
6.0 \\
6.5\end{array}$ & $\begin{array}{l}1991 \\
1992 \\
1993 \\
1994 \\
1995 \\
1996 \\
1997 \\
1998 \\
1999 \\
2000 \\
2001 \\
2002 \\
2003 \\
2004 \\
2005 \\
2006 \\
2007 \\
2008 \\
2009 \\
2010 \\
2011 \\
2012 \\
2013 \\
2014 \\
2015 \\
2016 \\
2017 \\
2018\end{array}$ & $\begin{array}{l} \\
36 \\
43 \\
53 \\
43 \\
57 \\
54 \\
54 \\
41 \\
40 \\
43 \\
62 \\
63 \\
60 \\
68 \\
67 \\
50 \\
44 \\
76 \\
78 \\
48 \\
51 \\
63 \\
75 \\
65 \\
53 \\
69 \\
57\end{array}$ & $\begin{array}{l}- \\
58 \\
38 \\
48 \\
43 \\
67 \\
60 \\
64 \\
33 \\
38 \\
50 \\
63 \\
54 \\
59 \\
59 \\
58 \\
41 \\
50 \\
79 \\
65 \\
52 \\
63 \\
60 \\
67 \\
67 \\
53 \\
52 \\
54\end{array}$ & $\begin{array}{l}- \\
63 \\
34 \\
49 \\
48 \\
51 \\
54 \\
54 \\
31 \\
38 \\
48 \\
55 \\
57 \\
58 \\
60 \\
40 \\
43 \\
59 \\
79 \\
57 \\
55 \\
57 \\
60 \\
68 \\
79 \\
60 \\
64\end{array}$ & $\begin{array}{l}4 \\
48 \\
50 \\
42 \\
53 \\
59 \\
50 \\
43 \\
42 \\
40 \\
49 \\
52 \\
54 \\
65 \\
58 \\
50 \\
47 \\
70 \\
78 \\
49 \\
58 \\
53 \\
67 \\
56 \\
63 \\
57 \\
54\end{array}$ & $\begin{array}{l}- \\
51 \\
41 \\
48 \\
47 \\
58 \\
54 \\
54 \\
37 \\
39 \\
48 \\
58 \\
57 \\
60 \\
61 \\
54 \\
45 \\
56 \\
78 \\
62 \\
53 \\
56 \\
62 \\
66 \\
68 \\
56 \\
60\end{array}$ \\
\hline
\end{tabular}




\begin{tabular}{|c|c|c|c|c|c|c|c|c|c|c|c|}
\hline Year & $\overline{I Q}$ & $\| \mathrm{Q}$ & $\mathrm{IIIQ}$ & $I V Q$ & Annual & Year & $\mathrm{IQ}$ & $\| \mathrm{IIQ}$ & $\mathrm{IIIQ}$ & $\overline{I V Q}$ & Annual \\
\hline $\begin{array}{l}\text { 49. Limit } \\
\text { and sen } \\
\text { 49. Or } \\
\text { матер } \\
\text { про }\end{array}$ & $\begin{array}{l}\text { ns to } \\
\text { ishe } \\
(\mathrm{s} \\
\text { иче } \\
\text { B, त } \\
\text { илен }\end{array}$ & $\begin{array}{l}\text { ductic } \\
\text { oduct } \\
\text { e of e } \\
\text { ipons } \\
\text { paбp } \\
\text { Tь }\end{array}$ & $\begin{array}{l}\text { short } \\
\text { orese } \\
\text { rprise } \\
\text { дства } \\
\text { тов, } \\
\text { рля п }\end{array}$ & $\begin{array}{l}\text { e of ral } \\
\text { situatio } \\
\% \text { ) } \\
\text { ехватк } \\
\text { ущая } \\
\text { mрият }\end{array}$ & $\begin{array}{l}\text { material } \\
\text { industry } \\
\text { сырья, } \\
\text { туация, } \\
(\%) \%\end{array}$ & $\begin{array}{r}50 . \text { Lin } \\
\text { reso } \\
50 . \\
\text { фин } \\
\text { прс }\end{array}$ & $\begin{array}{l}\text { tions } \\
\text { es, pr } \\
\text { рани } \\
\text { совы } \\
\text { шшле }\end{array}$ & $\begin{array}{l}\text { rodu } \\
\text { nt sit } \\
\text { nterp } \\
\text { uя חy } \\
\text { eyp } \\
\text { cTb }\end{array}$ & $\begin{array}{l}\text { n: sh } \\
\text { on, in } \\
\text { s, \% } \\
\text { воодс } \\
\text { теку } \\
\text { я пре }\end{array}$ & $\begin{array}{l}\text { gie of } \\
\text { stry (s } \\
\\
\text { : нехв } \\
\text { ситу } \\
\text { сияти }\end{array}$ & $\begin{array}{l}\text { nancial } \\
\text { are of } \\
\text { тка } \\
\text { ция, } \\
\% \text { ) }\end{array}$ \\
\hline 1991 & - & - & - & 82 & - & 1991 & - & - & - & 18 & - \\
\hline 1992 & 63 & 43 & 39 & 40 & 46 & 1992 & 29 & 46 & 50 & 56 & 45 \\
\hline 1993 & 33 & 27 & 19 & 14 & 23 & 1993 & 56 & 61 & 64 & 65 & 62 \\
\hline 1994 & 13 & 19 & 20 & 25 & 19 & 1994 & 69 & 66 & 57 & 62 & 64 \\
\hline 1995 & 24 & 21 & 25 & 19 & 22 & 1995 & 63 & 65 & 59 & 68 & 64 \\
\hline 1996 & 25 & 23 & 18 & 22 & 22 & 1996 & 66 & 78 & 68 & 69 & 70 \\
\hline 1997 & 25 & 21 & 24 & 27 & 24 & 1997 & 70 & 67 & 68 & 72 & 69 \\
\hline 1998 & 24 & 30 & 29 & 34 & 29 & 1998 & 70 & 68 & 70 & 67 & 69 \\
\hline 1999 & 38 & 37 & 42 & 30 & 37 & 1999 & 66 & 63 & 61 & 64 & 64 \\
\hline 2000 & 34 & 29 & 27 & 29 & 30 & 2000 & 63 & 60 & 59 & 62 & 61 \\
\hline 2001 & $\begin{array}{l}34 \\
26\end{array}$ & 22 & 20 & 27 & 24 & 2001 & 66 & 48 & 56 & 54 & 56 \\
\hline 2002 & 15 & 14 & 15 & 18 & 16 & 2002 & 58 & 54 & 56 & 52 & 55 \\
\hline 2003 & 15 & 18 & 17 & 12 & 16 & 2003 & 47 & 57 & 46 & 47 & 49 \\
\hline 2004 & 16 & 21 & 19 & 21 & 19 & 2004 & 51 & 48 & 47 & 40 & 47 \\
\hline & 13 & 12 & 12 & 17 & 14 & 2005 & 54 & 41 & 46 & 38 & 45 \\
\hline 2006 & 8 & 15 & 16 & 14 & 13 & 2006 & 41 & 39 & 45 & 34 & 40 \\
\hline 2007 & 19 & 14 & 22 & 18 & 18 & 2007 & 40 & 41 & 37 & 29 & 37 \\
\hline 2008 & 18 & 13 & 16 & 7 & 14 & 2008 & 34 & 36 & 41 & 47 & 39 \\
\hline 2009 & 11 & 9 & 17 & 14 & 13 & 2009 & 44 & 47 & 44 & 37 & 43 \\
\hline 2010 & 11 & 13 & 19 & 12 & 14 & 2010 & 46 & 40 & 43 & 37 & 42 \\
\hline 2011 & 14 & 17 & 11 & 10 & 13 & 2011 & 41 & 39 & 45 & 36 & 40 \\
\hline 2012 & 8 & 11 & 10 & 13 & 11 & 2012 & 35 & 33 & 26 & 31 & 31 \\
\hline 2013 & 10 & 7 & 10 & 7 & 9 & 2013 & 41 & 32 & 37 & 30 & 35 \\
\hline 2014 & 8 & 13 & 8 & 10 & 10 & 2014 & 33 & 30 & 21 & 37 & 30 \\
\hline 2015 & 7 & 5 & 10 & 11 & . & 5 & 30 & 35 & 33 & 37 & 34 \\
\hline 2016 & 4 & 12 & 13 & 6 & 9 & 2016 & 41 & 35 & 33 & 40 & 37 \\
\hline 2017 & 10 & 8 & 7 & 7 & 8 & 2017 & 33 & 22 & 36 & 33 & 31 \\
\hline 2018 & 9 & 8 & & & & 2018 & 23 & 24 & & & \\
\hline $\begin{array}{r}51 . L \\
\text { presen } \\
51 . \text { Orp } \\
\text { силы, }\end{array}$ & $\begin{array}{l}\text { ation } \\
\text { uatio } \\
\text { чен } \\
\text { щая }\end{array}$ & $\begin{array}{l}\text { уаци } \\
\text { еддр }\end{array}$ & тий, & tage o & $\begin{array}{l}\text { bour, } \\
\text { es, \%) } \\
\text { абочей } \\
\text { (доля }\end{array}$ & $\begin{array}{r}52 . \operatorname{Lim} \\
\text { and p } \\
52 . \\
\text { oбор } \\
\text { пр }\end{array}$ & $\begin{array}{l}\text { ons to } \\
\text { ises, } \\
\text { рани } \\
\text { ания } \\
\text { Ішле }\end{array}$ & $\begin{array}{l}\text { oduc } \\
\text { ustry } \\
\text { uя חy } \\
\text { omeu } \\
\text { cTb ( }\end{array}$ & $\begin{array}{l}\text { sho } \\
\text { are } \\
\text { Bводс } \\
\text { й, те } \\
\text { я пр }\end{array}$ & $\begin{array}{l}\text { ye of } \epsilon \\
\text { nterpr } \\
\text { : нex } \\
\text { цая } \mathrm{cl} \\
\text { рияти }\end{array}$ & $\begin{array}{l}\text { uipment } \\
\text { es, \%) } \\
\text { iтка } \\
\text { уация, } \\
\% \text { ) }\end{array}$ \\
\hline 1991 & - & - & - & 28 & - & 1991 & - & - & - & - & - \\
\hline & 10 & 8 & 6 & 6 & 8 & & 16 & 5 & 2 & 2 & 6 \\
\hline & 5 & 9 & 7 & 3 & 6 & & 6 & 7 & 7 & 0 & 5 \\
\hline & 4 & 7 & 5 & 3 & 5 & 4 & 3 & 0 & 2 & 4 & 2 \\
\hline & 4 & 6 & 5 & 2 & 4 & 5 & 4 & 3 & 3 & 2 & 3 \\
\hline & 0 & 2 & 2 & 0 & 1 & & 0 & 6 & 3 & 2 & 3 \\
\hline & 2 & 2 & 3 & 0 & 2 & & 3 & 3 & 3 & 5 & 4 \\
\hline & 2 & 2 & 1 & 3 & 2 & & 3 & 2 & 4 & 3 & 3 \\
\hline & 2 & 6 & 7 & 5 & 5 & 9 & 4 & 7 & 8 & 6 & 6 \\
\hline & 5 & 10 & 12 & 11 & 10 & & 8 & 9 & 8 & 10 & 9 \\
\hline & 7 & 6 & 8 & 10 & 8 & & 7 & 4 & 6 & 0 & 6 \\
\hline & 4 & 5 & 9 & 4 & 5 & & 6 & 4 & 8 & 6 & 6 \\
\hline & 6 & 8 & 10 & 10 & 8 & & 7 & 5 & 5 & 10 & 7 \\
\hline & 8 & 0 & 10 & 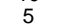 & 8 & & 9 & 10 & 6 & 6 & 8 \\
\hline & $\begin{array}{l}0 \\
4\end{array}$ & 12 & 12 & 8 & $\stackrel{\circ}{9}$ & & 8 & 7 & $\begin{array}{l}0 \\
6\end{array}$ & $\begin{array}{l}0 \\
6\end{array}$ & $\begin{array}{l}0 \\
7\end{array}$ \\
\hline 20 & 7 & 11 & 22 & 15 & 14 & 6 & 11 & 14 & 15 & 11 & 13 \\
\hline & 19 & 25 & 28 & 21 & 23 & & 12 & 10 & 12 & 11 & 11 \\
\hline & 25 & 20 & 25 & 6 & 19 & & 12 & 10 & 6 & 3 & 8 \\
\hline & 3 & 5 & 7 & 2 & 4 & 2009 & 3 & 3 & 3 & 5 & 4 \\
\hline & 4 & 11 & 13 & 5 & 8 & 2010 & 5 & 7 & 9 & 9 & 7 \\
\hline & 9 & 7 & 14 & 9 & 10 & & 8 & 7 & 9 & 12 & 9 \\
\hline & 9 & 12 & 17 & 10 & 12 & & 8 & 8 & 6 & 17 & 10 \\
\hline & 12 & 10 & 16 & 4 & 11 & ט & 6 & 12 & 6 & 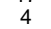 & 7 \\
\hline & 8 & 5 & 13 & 3 & 7 & & 8 & 5 & 6 & $\begin{array}{c}4 \\
10\end{array}$ & 7 \\
\hline 2 & 4 & 11 & 2 & 2 & 5 & 2 & 7 & 9 & 2 & 11 & 7 \\
\hline 201 & 4 & 9 & 4 & 9 & 6 & 2016 & 12 & 14 & 13 & 11 & 12 \\
\hline 2017 & 2 & 8 & 2 & 6 & 5 & 2017 & 14 & 12 & 21 & 9 & 14 \\
\hline 2 & 9 & 11 & & & & 2018 & 11 & 0 & & & \\
\hline
\end{tabular}


The Russian Economic Barometer, Vol. XXVII, 3/4, 2018

\begin{tabular}{|c|c|c|c|c|c|c|c|c|c|c|c|}
\hline Year & $I Q$ & $\| \mathrm{Q}$ & $\mathrm{IIIQ}$ & $I V Q$ & Annual & Year & $1 Q$ & $\| \mathrm{IIQ}$ & III Q & $\mathrm{IVQ}$ & Annual \\
\hline \multicolumn{6}{|c|}{$\begin{array}{l}\text { 53. Limitations to capital investments: shortage of } \\
\text { financial resources, present situation, industry } \\
\text { (share of enterprises, \%) } \\
\text { 53. Ограничения капитальных вложений: нехватка } \\
\text { финансовых ресурсов, текущая ситуация, } \\
\text { промышленность (доля предприятий, \%) }\end{array}$} & \multicolumn{6}{|c|}{$\begin{array}{l}\text { 54. Limitations to capital investments: high prices of } \\
\text { equipment and construction, present situation, } \\
\text { industry (share of enterprises, \%) } \\
\text { 54. Ограничения капитальных вложений: высокие } \\
\text { цены на оборудование и строительство, текущая } \\
\text { ситуация, промышленность (доля предприятий, \%) }\end{array}$} \\
\hline $\begin{array}{l}1992 \\
1993 \\
1994 \\
1995 \\
1996 \\
1997 \\
1998 \\
1999 \\
2000 \\
2001 \\
2002 \\
2003 \\
2004 \\
2005 \\
2006 \\
2007 \\
2008 \\
2009 \\
2010 \\
2011 \\
2012 \\
2013 \\
2014 \\
2015 \\
2016 \\
2017 \\
2018\end{array}$ & $\begin{array}{l}- \\
70 \\
54 \\
68 \\
70 \\
72 \\
74 \\
84 \\
82 \\
82 \\
85 \\
79 \\
74 \\
78 \\
79 \\
78 \\
73 \\
71 \\
83 \\
78 \\
69 \\
70 \\
68 \\
59 \\
66 \\
67 \\
58\end{array}$ & $\begin{array}{l}66 \\
72 \\
61 \\
63 \\
72 \\
74 \\
82 \\
81 \\
78 \\
81 \\
85 \\
82 \\
78 \\
78 \\
75 \\
74 \\
75 \\
83 \\
74 \\
74 \\
66 \\
64 \\
67 \\
57 \\
57 \\
58 \\
53\end{array}$ & $\begin{array}{l}72 \\
77 \\
70 \\
68 \\
67 \\
75 \\
81 \\
85 \\
83 \\
81 \\
85 \\
80 \\
83 \\
79 \\
77 \\
76 \\
75 \\
82 \\
77 \\
71 \\
69 \\
66 \\
74 \\
60 \\
66 \\
61 \\
46\end{array}$ & $\begin{array}{l}68 \\
76 \\
66 \\
75 \\
75 \\
71 \\
88 \\
80 \\
82 \\
80 \\
83 \\
78 \\
78 \\
76 \\
71 \\
68 \\
69 \\
83 \\
75 \\
77 \\
77 \\
75 \\
58 \\
59 \\
50 \\
46\end{array}$ & $\begin{array}{l}- \\
74 \\
63 \\
69 \\
71 \\
73 \\
81 \\
82 \\
81 \\
81 \\
84 \\
80 \\
78 \\
78 \\
76 \\
74 \\
73 \\
80 \\
77 \\
75 \\
70 \\
69 \\
67 \\
59 \\
60 \\
58\end{array}$ & $\begin{array}{l}1992 \\
1993 \\
1994 \\
1995 \\
1996 \\
1997 \\
1998 \\
1999 \\
2000 \\
2001 \\
2002 \\
2003 \\
2004 \\
2005 \\
2006 \\
2007 \\
2008 \\
2009 \\
2010 \\
2011 \\
2012 \\
2013 \\
2014 \\
2015 \\
2016 \\
2017 \\
2018\end{array}$ & $\begin{array}{l}- \\
67 \\
58 \\
63 \\
50 \\
48 \\
46 \\
50 \\
52 \\
56 \\
56 \\
44 \\
53 \\
48 \\
55 \\
64 \\
65 \\
57 \\
53 \\
54 \\
51 \\
43 \\
54 \\
52 \\
53 \\
53 \\
37\end{array}$ & $\begin{array}{l}73 \\
71 \\
55 \\
64 \\
52 \\
50 \\
42 \\
50 \\
59 \\
57 \\
54 \\
56 \\
50 \\
49 \\
52 \\
65 \\
63 \\
44 \\
54 \\
53 \\
50 \\
51 \\
44 \\
52 \\
49 \\
49 \\
35\end{array}$ & $\begin{array}{l}77 \\
77 \\
54 \\
60 \\
48 \\
51 \\
39 \\
62 \\
61 \\
59 \\
60 \\
56 \\
54 \\
52 \\
58 \\
61 \\
59 \\
54 \\
59 \\
52 \\
55 \\
48 \\
40 \\
56 \\
51 \\
43^{*} \\
38\end{array}$ & $\begin{array}{l}68 \\
69 \\
62 \\
50 \\
49 \\
50 \\
57 \\
53 \\
60 \\
60 \\
51 \\
54 \\
49 \\
55 \\
58 \\
63 \\
48 \\
49 \\
55 \\
57 \\
51 \\
50 \\
52 \\
45 \\
61 \\
66\end{array}$ & $\begin{array}{l}- \\
69 \\
57 \\
59 \\
50 \\
50 \\
46 \\
54 \\
58 \\
58 \\
55 \\
53 \\
52 \\
51 \\
56 \\
63 \\
59 \\
51 \\
55 \\
54 \\
52 \\
48 \\
48 \\
51 \\
53 \\
53\end{array}$ \\
\hline \multicolumn{6}{|c|}{$\begin{array}{l}\text { 55. Limitations to capital investments: high bank } \\
\text { interest, present situation, industry (share of } \\
\text { enterprises, \%) } \\
\text { 5. Ограничения капитальных вложений: высокий } \\
\text { банковский процент, текущая ситуация, } \\
\text { промышленность (доля предприятий, \%) }\end{array}$} & \multicolumn{6}{|c|}{$\begin{array}{l}\text { 56. Limitations to capital investments: high } \\
\text { indebtedness, present situation, industry (share of } \\
\text { enterprises, \%) } \\
\text { 56. Ограничения капитальных вложений: большая } \\
\text { задолженность, текущая ситуация, } \\
\text { промышленность (доля предприятий, \%) }\end{array}$} \\
\hline $\begin{array}{l}1992 \\
1993 \\
1994 \\
1995 \\
1996 \\
1997 \\
1998 \\
1999 \\
2000 \\
2001\end{array}$ & 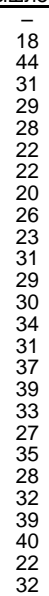 & $\begin{array}{l}33 \\
34 \\
29 \\
38 \\
31 \\
30 \\
35 \\
24 \\
40 \\
24\end{array}$ & $\begin{array}{l}37 \\
37 \\
33 \\
39 \\
44 \\
38 \\
50 \\
34 \\
26^{*} \\
26\end{array}$ & $\begin{array}{l}40 \\
30 \\
42 \\
41 \\
45 \\
35 \\
34\end{array}$ & $\begin{array}{l}32 \\
35 \\
36 \\
35 \\
42 \\
33 \\
31\end{array}$ & $\begin{array}{l}1992 \\
1993 \\
1994 \\
1995 \\
1996\end{array}$ & $\begin{array}{c}0 \\
7 \\
8 \\
11 \\
8\end{array}$ & $\begin{array}{c}21 \\
20 \\
19 \\
19 \\
15 \\
11 \\
12 \\
7 \\
9 \\
11 \\
11 \\
8 \\
11 \\
8 \\
7 \\
9 \\
6 \\
4 \\
12\end{array}$ & $\begin{array}{l}13 \\
13 \\
22 \\
29 \\
35 \\
42 \\
42 \\
38 \\
27 \\
23 \\
23 \\
21 \\
21 \\
17 \\
17 \\
13 \\
12 \\
12 \\
7 \\
9 \\
6 \\
13 \\
13 \\
16 \\
4 \\
5 \\
9 \\
6 \\
4\end{array}$ & $\begin{array}{c}18 \\
25 \\
29 \\
40 \\
32 \\
39 \\
31 \\
24 \\
23 \\
22 \\
19 \\
17 \\
18 \\
14 \\
9 \\
12 \\
8 \\
10 \\
15 \\
7 \\
13 \\
9 \\
5 \\
18 \\
11 \\
2\end{array}$ & $\begin{array}{c}- \\
22 \\
31 \\
36 \\
37 \\
40 \\
38 \\
27 \\
23 \\
22 \\
22 \\
17 \\
17 \\
13 \\
12 \\
8 \\
9 \\
9 \\
14 \\
11 \\
9 \\
8 \\
6 \\
9 \\
9 \\
7\end{array}$ \\
\hline
\end{tabular}




\begin{tabular}{|c|c|c|c|c|c|c|c|c|c|c|c|}
\hline \multirow{2}{*}{\multicolumn{6}{|c|}{ 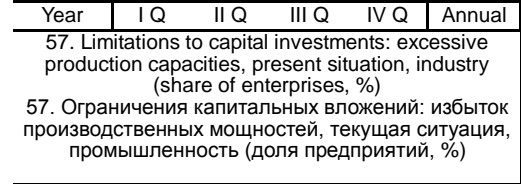 }} & Year & $I Q$ & $\| \mathrm{Q}$ & $\mathrm{IIIQ} \mathrm{Q}$ & $I V Q$ & Annual \\
\hline & & & & & & \multicolumn{6}{|c|}{$\begin{array}{l}\text { 58. Limitations to capital investments: insufficient } \\
\text { profitability of investment projects, present situation, } \\
\text { industry (share of enterprises, \%) } \\
\text { 58. Oгрраничения капитальных вложений: } \\
\text { недостаточная прибыльность инвестиционных } \\
\text { проектов, текущая ситуация, промышленность } \\
\text { (доля предприятий, \%) }\end{array}$} \\
\hline 1992 & 1 & 4 & $\begin{array}{l}6 \\
6\end{array}$ & 5 & & 1992 & - & - & $\overline{-}$ & $\bar{c}$ & - \\
\hline $\begin{array}{l}1993 \\
1994\end{array}$ & $\begin{array}{l}4 \\
9\end{array}$ & $\begin{array}{r}5 \\
12\end{array}$ & $\begin{array}{l}11 \\
14\end{array}$ & $\begin{array}{l}6 \\
11\end{array}$ & $\begin{array}{l}6 \\
12\end{array}$ & & $\overline{7}$ & $\overline{8}$ & 4 & $\begin{array}{l}6 \\
8\end{array}$ & $\overline{8}$ \\
\hline 1095 & 11 & 15 & $\begin{array}{l}14 \\
14\end{array}$ & 17 & 14 & & 7 & 7 & 7 & $\begin{array}{l}0 \\
4\end{array}$ & $\begin{array}{l}0 \\
6\end{array}$ \\
\hline 1 & 14 & 15 & 18 & 18 & 16 & 6 & 6 & 6 & 7 & 5 & 6 \\
\hline & 20 & 20 & 19 & 21 & 20 & 97 & 8 & 8 & 6 & 6 & 7 \\
\hline 1998 & 19 & 16 & 19 & 10 & 16 & 1998 & 4 & 4 & 2 & 5 & 4 \\
\hline 1999 & 20 & 15 & 12 & 15 & 16 & & 7 & 4 & 4 & 5 & 5 \\
\hline $\begin{array}{l}2000 \\
2001\end{array}$ & $\begin{array}{l}14 \\
13\end{array}$ & $\begin{array}{l}11 \\
9\end{array}$ & $\begin{array}{l}15 \\
14\end{array}$ & 11 & 13 & & 5 & 4 & 4 & 7 & 5 \\
\hline & $\begin{array}{r}13 \\
15\end{array}$ & 9 & $\begin{array}{l}14 \\
11\end{array}$ & $\begin{array}{l}12 \\
13\end{array}$ & & & $\begin{array}{l}6 \\
7\end{array}$ & $\begin{array}{l}9 \\
5\end{array}$ & 8 & $\begin{array}{l}6 \\
3 \\
3\end{array}$ & 7 \\
\hline 20 & $\begin{array}{l}15 \\
11\end{array}$ & $\begin{array}{l}13 \\
13\end{array}$ & $\begin{array}{c}11 \\
9\end{array}$ & $\begin{array}{l}13 \\
12\end{array}$ & $\begin{array}{l}13 \\
11\end{array}$ & $\begin{array}{l}2002 \\
2003\end{array}$ & 10 & $\begin{array}{l}5 \\
7\end{array}$ & $\begin{array}{l}5 \\
6\end{array}$ & $\begin{array}{c}3 \\
10\end{array}$ & $\begin{array}{l}5 \\
8\end{array}$ \\
\hline & 13 & 8 & 13 & 10 & 11 & & 11 & 16 & 10 & 18 & 14 \\
\hline 2005 & 11 & 13 & 16 & 9 & 12 & & 13 & 12 & 10 & 11 & 12 \\
\hline $\begin{array}{l}2006 \\
2007\end{array}$ & $\begin{array}{l}10 \\
7\end{array}$ & $\begin{array}{l}7 \\
9\end{array}$ & $\begin{array}{l}10 \\
12\end{array}$ & $\begin{array}{l}13 \\
10\end{array}$ & $\begin{array}{l}10 \\
10\end{array}$ & & $\begin{array}{l}11 \\
19\end{array}$ & $\begin{array}{l}11 \\
16\end{array}$ & $\begin{array}{l}13 \\
23\end{array}$ & $\begin{array}{l}16 \\
11\end{array}$ & $\begin{array}{l}13 \\
10\end{array}$ \\
\hline & & $\begin{array}{c}9 \\
10\end{array}$ & $\begin{array}{c}12 \\
7\end{array}$ & $\begin{array}{l}10 \\
13\end{array}$ & $\begin{array}{l}10 \\
10\end{array}$ & & $\begin{array}{l}19 \\
15\end{array}$ & $\begin{array}{l}16 \\
12\end{array}$ & $\begin{array}{l}23 \\
16\end{array}$ & $\begin{array}{l}15 \\
13\end{array}$ & $\begin{array}{l}18 \\
14\end{array}$ \\
\hline 2009 & $\begin{array}{l}9 \\
8\end{array}$ & $\begin{array}{c}10 \\
9\end{array}$ & 12 & $\begin{array}{l}13 \\
10\end{array}$ & $\begin{array}{l}10 \\
10\end{array}$ & 2000 & $\begin{array}{c}15 \\
7\end{array}$ & $\begin{array}{l}12 \\
13\end{array}$ & $\begin{array}{l}16 \\
14\end{array}$ & $\begin{array}{l}13 \\
12\end{array}$ & $\begin{array}{l}l_{14}^{4} \\
12\end{array}$ \\
\hline 2010 & 10 & 10 & 14 & 8 & 10 & & 14 & 8 & 14 & 13 & 12 \\
\hline 20 & 8 & 9 & 10 & 5 & 8 & & 12 & 19 & 17 & 8 & 14 \\
\hline & $\begin{array}{l}11 \\
11\end{array}$ & $\begin{array}{l}16 \\
13\end{array}$ & $\begin{array}{l}7 \\
10\end{array}$ & $\begin{array}{l}10 \\
13\end{array}$ & $\begin{array}{l}11 \\
11\end{array}$ & & $\begin{array}{l}17 \\
19\end{array}$ & $\begin{array}{c}5 \\
16\end{array}$ & $\begin{array}{l}16 \\
15\end{array}$ & & 13 \\
\hline & 10 & 11 & $\begin{array}{l}10 \\
11\end{array}$ & 8 & 16 & & 24 & $\begin{array}{l}10 \\
12\end{array}$ & 15 & & \\
\hline & 13 & 9 & 4 & $\begin{array}{l}0 \\
10\end{array}$ & 9 & & ${ }_{7}^{24}$ & 13 & 13 & & 16 \\
\hline & 15 & & & & 14 & & 23 & 20 & 19 & & 120 \\
\hline & 9 & 16 & $12^{*}$ & 10 & 12 & 2017 & 13 & 20 & 15 & 24 & $\begin{array}{l}20 \\
18\end{array}$ \\
\hline 2018 & $\begin{array}{l}9 \\
11\end{array}$ & $\begin{array}{l}16 \\
12\end{array}$ & 10 & & & 2018 & $\begin{array}{l}13 \\
16\end{array}$ & $\begin{array}{l}20 \\
15\end{array}$ & $\begin{array}{l}15 \\
10\end{array}$ & & \\
\hline \multirow{5}{*}{\multicolumn{6}{|c|}{$\begin{array}{l}\text { 61. Percentage of enterprises with production } \\
\text { capacities redundant against demand anticipated } \\
\text { in } 12 \text { months (industry) } \\
\text { 61. Доля предприятий с избыточными } \\
\text { произодственными мощностями относительно } \\
\text { ожидаемого через } 12 \text { месяевев спроса (\%, } \\
\text { промышленность) }\end{array}$}} & \multirow{5}{*}{\multicolumn{6}{|c|}{$\begin{array}{l}\text { 62. Percentage of enterprises with production } \\
\text { capacities insufficient against demand anticipated in } \\
12 \text { months (industry) } \\
\text { 62. Доля препприятий с недостаточными } \\
\text { произодственными мощностмии относительно } \\
\text { ожидаемого через } 12 \text { месяцев спроса (\%, } \\
\text { промышленность) }\end{array}$}} \\
\hline & & & & & & & & & & & \\
\hline & & & & & & & & & & & \\
\hline & & & & & & & & & & & \\
\hline & & & & & & & & & & & \\
\hline $\begin{array}{l}1993 \\
1994\end{array}$ & $\overline{41}$ & 51 & $\begin{array}{l}37 \\
43\end{array}$ & $\begin{array}{l}37 \\
45\end{array}$ & 45 & $\begin{array}{l}1993 \\
1994\end{array}$ & $\overline{15}$ & $\overline{9}$ & $\begin{array}{l}23 \\
11\end{array}$ & $\begin{array}{c}144 \\
9\end{array}$ & 11 \\
\hline & 49 & 51 & 52 & 5 & & & 7 & & 9 & 8 & \\
\hline & 56 & 56 & 65 & 60 & 5 & & 8 & 8 & 7 & 8 & 8 \\
\hline & 63 & 59 & 58 & 57 & $5 \varsigma$ & & 5 & 8 & 8 & 9 & 8 \\
\hline & $\begin{array}{l}60 \\
54\end{array}$ & $\begin{array}{l}61 \\
51\end{array}$ & $\begin{array}{l}68 \\
49\end{array}$ & $\begin{array}{l}53 \\
46\end{array}$ & $\begin{array}{l}60 \\
50\end{array}$ & & $\begin{array}{l}3 \\
9\end{array}$ & 4 & 3 & $\begin{array}{l}9 \\
9\end{array}$ & $\begin{array}{c}5 \\
10\end{array}$ \\
\hline & $\begin{array}{l}54 \\
46\end{array}$ & & $\begin{array}{l}49 \\
43\end{array}$ & 4 & & & & 12 & $\begin{array}{l}11 \\
112\end{array}$ & & $\begin{array}{l}10 \\
11\end{array}$ \\
\hline & $\begin{array}{l}40 \\
46\end{array}$ & 42 & $\begin{array}{l}45 \\
37\end{array}$ & $\begin{array}{l}44 \\
42\end{array}$ & 4 & & 10 & 10 & 10 & 9 & 10 \\
\hline & 46 & 41 & 42 & 42 & 43 & & 3 & 10 & 10 & 7 & 8 \\
\hline & 35 & 38 & 39 & 3 & 37 & & 10 & 11 & 14 & 12 & 12 \\
\hline & 36 & 40 & 33 & 39 & 37 & & 8 & 11 & 9 & $\pi$ & 10 \\
\hline & 33 & 40 & 42 & 40 & 39 & & 13 & 13 & 12 & 12 & 12 \\
\hline & & & $\begin{array}{l}28 \\
28\end{array}$ & & 31 & & $\begin{array}{l}14 \\
16\end{array}$ & & & & 15 \\
\hline & $\begin{array}{l}27 \\
21\end{array}$ & 25 & $\begin{array}{l}28 \\
26\end{array}$ & & $\begin{array}{l}26 \\
2 C\end{array}$ & & $\begin{array}{l}16 \\
10\end{array}$ & $\begin{array}{l}19 \\
11\end{array}$ & 16 & 1 & 17 \\
\hline & $\begin{array}{l}21 \\
42\end{array}$ & 38 & $\begin{array}{l}20 \\
47\end{array}$ & 3 & 40 & & 10 & $\begin{array}{l}111 \\
9\end{array}$ & $\begin{array}{l}6 \\
9\end{array}$ & 12 & 10 \\
\hline & 33 & 32 & 26 & 15 & $2 \mathrm{col}$ & & 10 & 9 & 8 & & \\
\hline 201 & 27 & 25 & 19 & 19 & 23 & & 11 & 12 & 11 & 16 & 12 \\
\hline $\begin{array}{l}201 \\
201\end{array}$ & $\begin{array}{l}27 \\
22\end{array}$ & $\begin{array}{l}25 \\
22\end{array}$ & $\begin{array}{l}28 \\
20\end{array}$ & 30 & $\begin{array}{l}27 \\
? 2\end{array}$ & & 8 & 14 & 19 & 9 & 12 \\
\hline & 23 & 22 & 20 & 2 & 22 & & $\begin{array}{c}11 \\
6\end{array}$ & 5 & & & \\
\hline & 28 & 10 & 16 & 2 & $\begin{array}{l}24 \\
24\end{array}$ & & $\begin{array}{l}6 \\
8\end{array}$ & 13 & $\begin{array}{l}16 \\
12\end{array}$ & $\begin{array}{l}100 \\
8\end{array}$ & 10 \\
\hline & 2 & 4 & 27 & 2 & $2 \varsigma$ & & 10 & 11 & 13 & 11 & 1 \\
\hline 201 & 18 & 24 & 31 & 25 & & & 18 & 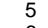 & 9 & 8 & \\
\hline & 18 & 28 & 22 & & & 2018 & & & & & \\
\hline
\end{tabular}


The Russian Economic Barometer, Vol. XXVII, 3/4, 2018

\begin{tabular}{|c|c|c|c|c|c|c|c|c|c|c|c|}
\hline Year & $I Q$ & $\| \mathrm{II}$ & $\mathrm{IIIQ}$ & $I V Q$ & Annual & Year & $1 \mathrm{IQ}$ & $\| \mathrm{IIQ}$ & $\mathrm{IIIQ}$ & $\mathrm{IVQ}$ & Annual \\
\hline \multicolumn{6}{|c|}{$\begin{array}{l}\text { 63. Percentage of enterprises with manpower } \\
\text { redundant against demand anticipated in } 12 \text { months } \\
\text { (industry) } \\
\text { 63. Доля предприятий с избыточной } \\
\text { укомплектованностью рабочей силой } \\
\text { относительно ожидаемого через 12 месяцев спроса } \\
\text { (\%, промышленность) }\end{array}$} & \multicolumn{6}{|c|}{$\begin{array}{l}\text { 64. Percentage of enterprises with manpower } \\
\text { insufficient against demand } \\
\text { anticipated in } 12 \text { months (industry) } \\
\text { 64. Доля предприятий с недостаточной } \\
\text { укомплектованностью рабочей силой } \\
\text { относительно ожидаемого через } 12 \text { месяцев } \\
\text { спроса (\%, промышленность) }\end{array}$} \\
\hline 1993 & - & - & 25 & 22 & - & 1993 & - & - & 24 & 21 & \\
\hline 1994 & 26 & 34 & 26 & 26 & 28 & 1994 & 19 & 18 & 25 & 20 & 20 \\
\hline 1995 & 24 & 27 & 22 & 35 & 27 & 1995 & 24 & 18 & 21 & 13 & 19 \\
\hline 1996 & 37 & 35 & 39 & 38 & 37 & 1996 & 12 & 12 & 11 & 10 & 11 \\
\hline 1997 & 39 & 39 & 34 & 38 & 38 & 1997 & 10 & 9 & 14 & 8 & 10 \\
\hline 1998 & 33 & 34 & 45 & 30 & 36 & 1998 & 10 & 6 & 10 & 13 & 10 \\
\hline 1999 & 27 & 19 & 14 & 15 & 19 & 1999 & 14 & 16 & 16 & 21 & 17 \\
\hline 2000 & 22 & 18 & 15 & 14 & 17 & 2000 & 16 & 21 & 25 & 26 & 22 \\
\hline 2001 & 13 & 13 & 12 & 14 & 13 & 2001 & 25 & 28 & 30 & 25 & 27 \\
\hline 2002 & 20 & 16 & 16 & 17 & 17 & 2002 & 22 & 25 & 25 & 18 & 23 \\
\hline 2003 & 12 & 14 & 10 & 10 & 12 & 2003 & 25 & 26 & 24 & 26 & 25 \\
\hline 2004 & 17 & 9 & 13 & 19 & 14 & 2004 & 19 & 26 & 28 & 19 & 23 \\
\hline 2005 & 14 & 17 & 12 & 13 & 14 & 2005 & 21 & 25 & 22 & 25 & 23 \\
\hline 2006 & 10 & 11 & 11 & 5 & 9 & 2006 & 23 & 32 & 35 & 35 & 31 \\
\hline 2007 & 7 & 3 & 9 & 5 & 6 & 2007 & 34 & 48 & 44 & 34 & 40 \\
\hline 2008 & 5 & 7 & 12 & 27 & 13 & 2008 & 39 & 39 & 38 & 14 & 33 \\
\hline 2009 & 25 & 19 & 22 & 15 & 20 & 2009 & 14 & 18 & 17 & 17 & 17 \\
\hline 2010 & 17 & 13 & 7 & 9 & 12 & 2010 & 18 & 27 & 25 & 24 & 23 \\
\hline 2011 & 10 & 9 & 8 & 4 & 8 & 2011 & 26 & 23 & 35 & 30 & 28 \\
\hline 2012 & 6 & 6 & 8 & 13 & 8 & 2012 & 23 & 18 & 32 & 25 & 25 \\
\hline 20 & 11 & 12 & 10 & 7 & 10 & 2013 & 26 & 25 & 24 & 23 & 25 \\
\hline 2014 & 11 & 7 & 8 & 9 & 9 & 2014 & 16 & 20 & 22 & 35 & 23 \\
\hline 2015 & 4 & 18 & 10 & 6 & 9 & 2015 & 14 & 16 & 22 & 18 & 18 \\
\hline 2016 & 14 & 20 & 9 & 10 & 13 & 2016 & 20 & 20 & 18 & 19 & 19 \\
\hline 2017 & 5 & 15 & 16 & 5 & 10 & 2017 & 18 & 17 & 19 & 13 & 17 \\
\hline 2018 & 8 & 19 & 14 & & & 2018 & 21 & 13 & 14 & & \\
\hline \multicolumn{6}{|c|}{$\begin{array}{l}\text { 65. Percentage of loss-making enterprises in the } \\
\text { previous half-year (industry) } \\
\text { 65. Доля предприятий убыточных за } \\
\text { предшествующие полгода (\%, промышленность) }\end{array}$} & \multicolumn{6}{|c|}{$\begin{array}{l}\text { 66. Percentage of enterprises likely to go bankrupt } \\
\text { in the coming } 1 \text { to } 2 \text { years (industry) } \\
\text { 66. Доля предприяяий, для которых угроза } \\
\text { обанкротиться в ближайшие } 1-2 \text { года выглядит } \\
\text { реальной (\%, промышленность) }\end{array}$} \\
\hline 1992 & - & - & - & - & - & 1992 & - & - & 27 & 24 & \\
\hline 1993 & - & 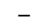 & - & - & - & 1993 & 14 & 21 & 27 & 22 & 21 \\
\hline & 11 & 17 & 25 & 23 & 19 & & 27 & 30 & 33 & 32 & 30 \\
\hline & 27 & 21 & 16 & 21 & 21 & 5 & 30 & 28 & 25 & 27 & 28 \\
\hline & 27 & 32 & 38 & 38 & 34 & & 37 & 36 & 39 & 45 & 39 \\
\hline & 39 & 44 & 45 & 33 & 40 & & 3 & 35 & 37 & 29 & 35 \\
\hline & 37 & 41 & 47 & 48 & 43 & & 3 & 43 & 41 & 42 & 40 \\
\hline & 40 & 31 & 32 & ? & 3 & & 3 & 26 & 24 & 22 & 27 \\
\hline & 26 & 25 & 28 & 2 & 26 & & 2 & 21 & 21 & 21 & 22 \\
\hline & 22 & 21 & 22 & 23 & 22 & & 20 & 18 & 15 & 15 & 17 \\
\hline & 27 & 29 & 36 & 27 & 3 & & 11 & 1 & 19 & 15 & 17 \\
\hline & 30 & 29 & 39 & 2 & 30 & & 17 & 16 & 14 & 8 & 14 \\
\hline 20 & 30 & 30 & 27 & 2 & 27 & & 14 & 19 & 15 & 13 & 15 \\
\hline & 21 & 26 & ? & 2 & 2 & & 10 & 11 & 13 & 8 & 11 \\
\hline & 18 & 23 & 22 & 21 & 21 & & 0 & 9 & 2 & 3 & 6 \\
\hline & 15 & 17 & 18 & 1 & 17 & & 2 & 5 & 3 & 4 & 4 \\
\hline & 13 & 14 & 18 & & & & 6 & 2 & 3 & 10 & 5 \\
\hline & 28 & 41 & 40 & 3 & 3 & & 7 & 13 & 12 & 15 & 13 \\
\hline 20 & 28 & 41 & 30 & 2 & 0 & & 7 & 9 & 7 & 8 & 8 \\
\hline & 26 & 27 & 35 & & 27 & & 9 & 10 & 8 & 11 & 9 \\
\hline & 15 & 18 & 15 & 18 & 17 & & 9 & 7 & 4 & 1 & 5 \\
\hline 20 & 0 & 16 & 1 & 17 & 4 & & 9 & 9 & 12 & 7 & 9 \\
\hline & 13 & 17 & 25 & 19 & 18 & & 10 & 8 & 6 & 4 & 7 \\
\hline 20 & 17 & 23 & 25 & 25 & 22 & & 5 & 13 & 8 & 11 & 9 \\
\hline 20 & 23 & 38 & 25 & 20 & 2 & & 9 & 6 & 2 & 2 & 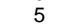 \\
\hline 20 & 11 & 32 & 18 & 23 & 21 & 2017 & 5 & 10 & 5 & 3 & 6 \\
\hline 2018 & 17 & 15 & 21 & & & 2018 & 5 & 3 & 6 & & \\
\hline
\end{tabular}




\begin{tabular}{|c|c|c|c|c|c|c|c|c|c|c|c|}
\hline Year & $\mathrm{IQ}$ & $\| \mathrm{II}$ & $\mathrm{IIIQ}$ & $\overline{I V Q}$ & Annual & Year & $\mathrm{IQ}$ & $\| \mathrm{Q}$ & $\mathrm{IIIQ}$ & IV Q & Annual \\
\hline \multicolumn{6}{|c|}{$\begin{array}{l}\text { 67. Job creation rate, industry, actual (6-month spans, } \\
\text { \%) } \\
\text { 67. Коэффиццент создания рабочих мест, } \\
\text { промышленность, фактические изменения за } 6 \\
\text { месяцев (\%) }\end{array}$} & \multicolumn{6}{|c|}{$\begin{array}{l}\text { 68. Job destruction rate, industry, actual (6-month } \\
\text { spans, \%) } \\
\text { 68. Коэффициент ликвидации рабочих мест, } \\
\text { промышленность, фактические изменения за } 6 \\
\text { месяцев (\%) }\end{array}$} \\
\hline 1993 & 0.5 & 0.9 & $\frac{8(10)}{1.2}$ & 0.9 & 0.9 & 1993 & 6.7 & 5.5 & 4.9 & 5.2 & 5.6 \\
\hline 1994 & 0.5 & 0.3 & 0.4 & 0.9 & 0.5 & 1994 & 7.6 & 9.3 & 8.8 & 6.7 & 8.1 \\
\hline 1995 & 1.1 & 1.4 & 1.2 & 0.7 & 1.1 & 1995 & 6.1 & 5.7 & 4.2 & 4.3 & 5.1 \\
\hline 1996 & 0.4 & 0.8 & 0.8 & 0.6 & 0.6 & 1996 & 4.9 & 5.0 & 5.2 & 5.3 & 5.1 \\
\hline 1997 & 0.7 & 1.0 & 1.5 & 0.9 & 1.0 & 1997 & 5.8 & 3.7 & 3.7 & 3.7 & 4.2 \\
\hline 1998 & 0.4 & 0.6 & 0.3 & 0.7 & 0.5 & 1998 & 4.0 & 3.7 & 3.7 & 3.3 & 3.7 \\
\hline 1999 & 1.2 & 2.2 & 2.2 & 1.6 & 1.8 & 1999 & 2.6 & 2.5 & 2.1 & 2.0 & 2.3 \\
\hline 2000 & 2.2 & 2.3 & 2.0 & 1.8 & 2.1 & 2000 & 2.0 & 1.7 & 1.2 & 1.9 & 1.7 \\
\hline 2001 & 1.6 & 1.8 & 1.5 & 1.3 & 1.6 & 2001 & 2.3 & 3.0 & 3.2 & 2.9 & 2.8 \\
\hline 2002 & 1.4 & 1.3 & 1.5 & 1.2 & 1.3 & 2002 & 2.2 & 3.2 & 2.7 & 3.5 & 2.9 \\
\hline 2003 & 0.9 & 1.4 & 1.0 & 1.2 & 1.1 & 2003 & 2.9 & 3.0 & 2.7 & 3.3 & 3.0 \\
\hline 2004 & 1.3 & 1.0 & 1.2 & 0.8 & 1.1 & 2004 & 3.2 & 3.1 & 2.9 & 2.9 & 3.0 \\
\hline 2005 & 0.7 & 1.0 & 1.3 & 0.8 & 1.0 & 2005 & 3.0 & 3.7 & 3.0 & 2.8 & 3.1 \\
\hline 2006 & 0.9 & 1.0 & 1.1 & 1.6 & 1.1 & 2006 & 2.4 & 3.2 & 1.9 & 1.7 & 2.3 \\
\hline 2007 & 1.9 & 2.0 & 1.7 & 1.6 & 1.8 & 2007 & 1.5 & 1.8 & 3.0 & 2.0 & 2.1 \\
\hline 2008 & 1.5 & 0.9 & 0.5 & 0.6 & 0.9 & 2008 & 1.4 & 3.5 & 3.8 & 5.8 & 3.6 \\
\hline 2009 & 0.3 & 1.0 & 0.5 & 1.1 & 0.7 & 2009 & 7. & 7.2 & 5.2 & 4. & 6.0 \\
\hline 2010 & 0.6 & 1.2 & 1.2 & 0.7 & 0.9 & 2010 & 5. & 3.8 & 3.2 & 2.9 & 3.8 \\
\hline 2011 & 1.4 & 2.1 & 1.1 & 0.9 & 1.4 & 2011 & 2. & 2.9 & 1.9 & 1.9 & 2.2 \\
\hline 2012 & 1.3 & 0.9 & 1.2 & 1.2 & 1.2 & 2012 & 2. & 3.0 & 1.5 & 1.9 & 2.2 \\
\hline 2013 & 1.2 & 0.8 & 0.5 & 0.3 & 0.7 & 2013 & 2. & 3.3 & 4.5 & 3. & 3.4 \\
\hline 2014 & 0.6 & 0.8 & 1.0 & 1.1 & 0. & 2014 & 2. & 2.7 & 2.1 & 3. & 2.7 \\
\hline 2015 & 1.2 & 0.9 & 0.8 & 0.5 & 0.9 & 2015 & 4.3 & 3.0 & 2.7 & 2.7 & 3.2 \\
\hline 2016 & 0.9 & 1.2 & 0.5 & 1.4 & 1.0 & 2016 & 4. & 2.7 & 1.4 & 2.6 & 2.7 \\
\hline 2017 & 1.4 & 1.0 & 1.1 & 1.6 & 1.3 & 2017 & 2.5 & 2.8 & 2.9 & 3.0 & 2.8 \\
\hline 2018 & 1.0 & 1.5 & & & & 2018 & 2.5 & 1.8 & & & \\
\hline \multicolumn{6}{|c|}{$\begin{array}{l}\text { 69. Job creation rate, industry, anticipated } \\
\text { (6-month spans, \%) } \\
\text { 69. Коэффрициент создания рабочих мест, } \\
\text { ромышленность, ожидаемые изменения за } 6 \\
\text { месяцев (\%) }\end{array}$} & \multicolumn{6}{|c|}{$\begin{array}{c}\text { 70. Job destruction rate, industry, anticipated } \\
\text { (6-month spans, \%) } \\
\text { 70. Коэфффициент ликвидации рабочих мест, } \\
\text { промышленность, ожидаемые изменения за } 6 \\
\text { месяцев (\%) }\end{array}$} \\
\hline 1993 & - & - & 0.7 & 0.9 & - & 1993 & - & - & 4.6 & 4.7 & - \\
\hline 1994 & 1.9 & 0.7 & 07 & 0.3 & 0.9 & 1994 & 4.4 & 5.9 & & 9.5 & 7.0 \\
\hline 1995 & 0.8 & 1.0 & 1.3 & 1.0 & 1.0 & 1995 & 7.4 & 6.2 & 3.6 & 5. & 5.6 \\
\hline 1996 & 0.6 & 0.9 & 0.5 & 0.5 & 0. & 1996 & 4. & 3.8 & 48 & 4. & 4.5 \\
\hline 1997 & 0.5 & 0.6 & 0.8 & 0.5 & 0.6 & 1997 & 5. & 5.2 & & 4 & 4.7 \\
\hline 1998 & 0.9 & 0.8 & 0.6 & 0.2 & 0. & 1998 & 3. & 2.6 & 3 & 3. & 3.5 \\
\hline 1999 & 0.3 & 1.0 & 0.8 & 1.5 & 0 & 1999 & 4. & 2.9 & & 1. & 2.7 \\
\hline 2000 & 1.7 & 1.8 & 1.8 & 1.8 & 1.8 & 2000 & 1. & 1.3 & 1.5 & 1.4 & 1.5 \\
\hline 2 & 2.2 & 9 & 1.6 & 1.2 & & & 0. & 14 & & 2 & 1.6 \\
\hline 2002 & 1.2 & 1.7 & 1.3 & 1.0 & 1. & 2002 & 1. & 1 & 1 & 2 & 1.9 \\
\hline & 1.3 & 15 & 1.4 & 1. & & & & 2 & & 1 & 2.0 \\
\hline 2004 & 1.4 & 1.8 & 1.2 & 0.8 & 1. & & 2. & 2 & & 2 & 2.4 \\
\hline & 1.2 & 1.5 & 0.9 & 1.1 & 1 & & 3 & 1 & 2 & 2 & 2.5 \\
\hline 2006 & 0.9 & 1.1 & 1. & 1.3 & & & 2. & 1 & & 2 & 2.1 \\
\hline 2007 & 1.5 & 1.8 & 1.1 & 1.2 & 1. & 2007 & 1.5 & 1.1 & 1.4 & 1.5 & 1.4 \\
\hline 2 & 1.3 & & 1. & 1.0 & & & 2. & & & & 1.9 \\
\hline 2009 & 0.9 & 0.7 & 0.9 & 0.8 & 0. & & 2. & 5 & & 4 & 4.6 \\
\hline & 0.5 & 0.6 & 1. & 0.9 & & & 3. & 2 & & & 2.5 \\
\hline 2011 & 1.0 & 1.0 & 1.3 & 1.1 & 1. & & 2. & 2. & & 2. & 2.3 \\
\hline 2012 & 0.9 & 1.2 & 1.6 & 0.8 & 1. & 20 & 2. & 1.8 & 1.2 & 2. & 1.9 \\
\hline 2013 & 1.4 & 1.9 & 1.6 & 0.7 & 1 & & 1. & 1. & 1.5 & 2 & 1.8 \\
\hline 2 & 1. & 7 & 1 & 0 & 1 & & 2. & 2 & 1 & 2 & 2.0 \\
\hline & 0.8 & 1.1 & 0.8 & 0.8 & 0 & & 1. & 2. & 3 & 3. & 2.7 \\
\hline 2016 & 0.4 & 0.6 & 0.5 & 0.7 & 0.6 & 2016 & 1.9 & 2.8 & 2.9 & 2.7 & 2.6 \\
\hline 2017 & 0.7 & 1.1 & 0.6 & 0.6 & 0.7 & 2017 & 2. & 1.9 & 1.5 & 1.5 & 1.8 \\
\hline 2018 & 1.1 & 1.0 & 0.5 & 0.7 & 0.8 & 2018 & 2.2 & 2.4 & 2.2 & 1.2 & 2.0 \\
\hline
\end{tabular}


The Russian Economic Barometer, Vol. XXVII, 3/4, 2018

\begin{tabular}{|c|c|c|c|c|c|c|c|c|c|c|c|}
\hline Year & $I Q$ & $\| \mathrm{II}$ & III Q & IV Q & Annual & Year & $\mathrm{IQ}$ & $\| \mathrm{QQ}$ & III Q & $I V Q$ & Annual \\
\hline \multicolumn{6}{|c|}{$\begin{array}{l}\text { 71. Portion of loss-making production in the total } \\
\text { output, industry (\%) } \\
\text { 71. Доля убыточной продукции в выпуске, } \\
\text { промышленность (\%) }\end{array}$} & \multicolumn{6}{|c|}{$\begin{array}{l}\text { 72. Share of enterprises producing loss-making } \\
\text { output, industry (\%) } \\
\text { 72. Доля предприятий, выпускающих убыточную } \\
\text { продукцию, промышленность (\%) }\end{array}$} \\
\hline 1994 & - & - & 10 & 9 & - & 1994 & - & - & 61 & 60 & - \\
\hline 1995 & 10 & 13 & 9 & 10 & 10 & 1995 & 63 & 55 & 64 & 62 & 61 \\
\hline 1996 & 11 & 14 & 14 & 13 & 13 & 1996 & 71 & 74 & 72 & 74 & 73 \\
\hline 1997 & 15 & 15 & 15 & 14 & 15 & 1997 & 70 & 78 & 74 & 78 & 75 \\
\hline 1998 & 16 & 17 & 19 & 17 & 17 & 1998 & 75 & 85 & 83 & 82 & 81 \\
\hline 1999 & 17 & 17 & 14 & 16 & 16 & 1999 & 78 & 77 & 76 & 76 & 77 \\
\hline 2000 & 14 & 12 & 12 & 14 & 13 & 2000 & 77 & 74 & 73 & 77 & 75 \\
\hline 2001 & 12 & 14 & 11 & 12 & 12 & 2001 & 71 & 72 & 72 & 75 & 73 \\
\hline 2002 & 13 & 12 & 12 & 13 & 12 & 2002 & 82 & 79 & 79 & 85 & 81 \\
\hline 2003 & 13 & 13 & 11 & 12 & 12 & 2003 & 78 & 83 & 74 & 75 & 78 \\
\hline 2004 & 14 & 14 & 13 & 13 & 13 & 2004 & 75 & 79 & 75 & 75 & 76 \\
\hline 2005 & 13 & 14 & 12 & 15 & 13 & 2005 & 76 & 74 & 79 & 78 & 77 \\
\hline 2006 & 13 & 15 & 9 & 15 & 13 & 2006 & 75 & 79 & 72 & 81 & 77 \\
\hline 2007 & 13 & 11 & 13 & 11 & 12 & 2007 & 82 & 79 & 77 & 73 & 78 \\
\hline 2008 & 10 & 11 & 12 & 13 & 12 & 2008 & 78 & 74 & 75 & 81 & 77 \\
\hline 2009 & 17 & 19 & 18 & 17 & 18 & 2009 & 87 & 84 & 81 & 83 & 84 \\
\hline 2010 & 17 & 18 & 17 & 18 & 18 & 2010 & 83 & 88 & 82 & 82 & 84 \\
\hline 2011 & 15 & 16 & 13 & 13 & 14 & 2011 & 84 & 84 & 77 & 83 & 82 \\
\hline 2012 & 13 & 11 & 11 & 13 & 12 & 2012 & 80 & 78 & 84 & 76 & 80 \\
\hline 2013 & 10 & 10 & 10 & 10 & 10 & 2013 & 80 & 84 & 70 & 76 & 78 \\
\hline 2014 & 9 & 10 & 7 & 10 & 9 & 2014 & 74 & 78 & 76 & 73 & 75 \\
\hline 2015 & 13 & 12 & 7 & 9 & 10 & 2015 & 80 & 67 & 72 & 74 & 73 \\
\hline 2016 & 10 & 12 & 11 & 10 & 11 & 2016 & 69 & 70 & 73 & 76 & 72 \\
\hline 2017 & 8 & 10 & 8 & 7 & 8 & 2017 & 68 & 70 & $68^{*}$ & 68 & 69 \\
\hline 2018 & 6 & 10 & 17 & & & 2018 & 63 & 85 & 77 & & \\
\hline \multicolumn{6}{|c|}{$\begin{array}{l}\text { 73. Proportion of costs recovered in selling prices for } \\
\text { loss-making output, industry (\%) } \\
\text { 73. Степень окупаемости убыточной продукции, } \\
\text { промышленность (\%) }\end{array}$} & \multicolumn{6}{|c|}{$\begin{array}{l}\text { 74. Loss ratio: portion of losses from loss-making } \\
\text { output in total costs, industry (\%) } \\
\text { 74. Доля убытков от убыточной продукции в } \\
\text { общих издержках, промышленность (\%) }\end{array}$} \\
\hline 1995 & - & - & - & 74 & - & 1995 & - & - & - & 2.6 & $\begin{array}{c}- \\
34\end{array}$ \\
\hline $\begin{array}{l}1996 \\
1997\end{array}$ & $\begin{array}{l}75 \\
72\end{array}$ & $\begin{array}{l}75 \\
73\end{array}$ & $\begin{array}{l}73 \\
76\end{array}$ & $\begin{array}{l}75 \\
74\end{array}$ & $\begin{array}{l}75 \\
74\end{array}$ & $\begin{array}{l}1996 \\
1997\end{array}$ & $\begin{array}{l}2.8 \\
4.2\end{array}$ & $\begin{array}{l}3.5 \\
4.0\end{array}$ & $\begin{array}{l}3.8 \\
3.6\end{array}$ & $\begin{array}{l}3.3 \\
3.6\end{array}$ & $\begin{array}{l}3.4 \\
3.8\end{array}$ \\
\hline 1998 & 74 & 73 & 73 & 74 & 74 & 1998 & 4.2 & 4.6 & 5.0 & 4.4 & 4.6 \\
\hline 1999 & 72 & 73 & 73 & 74 & 73 & 1999 & 4.8 & 4.5 & 3.9 & 4.0 & 4.3 \\
\hline 2000 & 76 & 76 & 75 & 75 & 76 & 2000 & 3.3 & 3.0 & 3.0 & 3.5 & 3.2 \\
\hline 2001 & 76 & 75 & 75 & 74 & 75 & 2001 & 3.0 & 3.6 & 2.7 & 3.2 & 3.1 \\
\hline 2002 & 74 & 76 & 79 & 76 & 76 & 2002 & 3.4 & 2.9 & 2.5 & 3.1 & 3.0 \\
\hline 2003 & 77 & 75 & 73 & 75 & 75 & 2003 & 3.0 & 3.2 & 3.1 & 3.1 & 3.1 \\
\hline 2004 & 71 & 80 & 73 & 76 & 75 & 2004 & 4.0 & 2.7 & 3. & 3.0 & 3.3 \\
\hline 2005 & 75 & 76 & 83 & 78 & 78 & 2005 & 3.1 & 3.4 & 2. & 3.3 & 3.0 \\
\hline 2006 & 77 & 78 & 80 & 78 & 78 & 2006 & 3.0 & 3.2 & 1.8 & 3.3 & 2.8 \\
\hline 2007 & 78 & 75 & 72 & 77 & 76 & 2007 & 2.9 & 2.8 & 3.6 & 2.6 & 3.0 \\
\hline 2008 & 77 & 74 & 78 & 76 & 76 & 2008 & 2.3 & 2.8 & 2.6 & 3.1 & 2.7 \\
\hline 2009 & 70 & 73 & 74 & 79 & 74 & 2009 & 5.1 & 5.0 & 4.6 & 3.6 & 4.6 \\
\hline 2010 & 76 & 76 & 77 & 72 & 75 & 2010 & 4.1 & 4.3 & 4. & 5.0 & 4.4 \\
\hline 2011 & 74 & 72 & 73 & 73 & 73 & 2011 & 4.0 & 4.5 & 3.6 & 2.2 & 3.6 \\
\hline 2012 & 74 & 69 & 82 & 78 & 76 & 2012 & 3.4 & 3.5 & 2.0 & 2.8 & 2.9 \\
\hline 2013 & 74 & 75 & 74 & 68 & 73 & 2013 & 2.7 & 2.5 & 2.7 & 2.3 & 2.6 \\
\hline 2014 & 77 & 73 & 78 & 73 & 75 & 2014 & 2.0 & 2.7 & 1.6 & 2.8 & 2.3 \\
\hline 2015 & 71 & 74 & 75 & 66 & 72 & 2015 & 3.8 & 3.1 & 1.6 & 3.0 & 2.9 \\
\hline 2016 & 77 & 75 & 74 & 77 & 76 & 2016 & 2.4 & 2.9 & 2.8 & $2.3^{*}$ & $2.6^{*}$ \\
\hline 2017 & 75 & 77 & 65 & 75 & 73 & 2017 & 2.1 & 2.2 & 3.1 & 1.9 & 2.3 \\
\hline 2018 & 71 & 69 & 72 & & & 2018 & 1.7 & 3.1 & 4.8 & & \\
\hline
\end{tabular}




\begin{tabular}{|c|c|c|c|c|c|c|c|c|c|c|c|}
\hline Year & $\mathrm{IQ}$ & $\mathrm{IIQ}$ & III Q & $I V Q$ & Annual & Year & $\mathrm{IQ}$ & $\mathrm{IIQ}$ & $\mathrm{III} Q$ & IV Q & Annual \\
\hline \multicolumn{6}{|c|}{$\begin{array}{l}\text { 175. Reasons for manufacturing loss-making } \\
\text { production: efforts to keep enterprises' market share, } \\
\text { industry (\%) } \\
\text { 175. Причины производства убыточной продукции: } \\
\text { стремление удержать свою долю на прежних } \\
\text { рынках сбыта, промышленность (\%) }\end{array}$} & \multicolumn{6}{|c|}{ 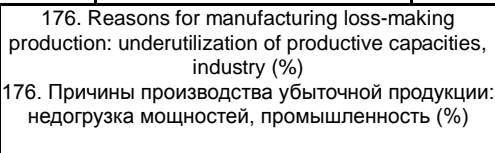 } \\
\hline 1995 & - & - & 21 & 19 & - & 1995 & - & - & - & - & - \\
\hline 1996 & 24 & 27 & 30 & 39 & 30 & 1996 & - & - & - & - & - \\
\hline 1997 & 35 & 39 & 38 & 44 & 39 & 1997 & - & - & - & - & - \\
\hline 1998 & 34 & 33 & 37 & 35 & 35 & 1998 & 44 & 45 & 43 & 45 & 44 \\
\hline 1999 & 35 & 29 & 33 & 33 & 33 & 1999 & 43 & 42 & 42 & 39 & 42 \\
\hline 2000 & 34 & 31 & 29 & 31 & 31 & 2000 & 38 & 38 & 34 & 44 & 39 \\
\hline 2001 & 31 & 32 & 27 & 33 & 31 & 2001 & 35 & 34 & 35 & 45 & 37 \\
\hline 2002 & 33 & 39 & 39 & 42 & 38 & 2002 & 33 & 45 & 49 & 33 & 40 \\
\hline 2003 & 38 & 39 & 36 & 39 & 38 & 2003 & 35 & 46 & 28 & 28 & 34 \\
\hline 2004 & 41 & 45 & 39 & 40 & 41 & 2004 & 33 & 28 & 27 & 27 & 29 \\
\hline 2005 & 34 & 36 & 41 & 43 & 39 & 2005 & 33 & 30 & 33 & 32 & 32 \\
\hline 2006 & 45 & 37 & 37 & 42 & 40 & 2006 & 24 & 27 & 27 & 32 & 28 \\
\hline 2007 & 42 & 44 & 46 & 40 & 43 & 2007 & 30 & 32 & 24 & 21 & 27 \\
\hline 2008 & 39 & 33 & 38 & 44 & 39 & 2008 & 25 & 25 & 21 & 22 & 23 \\
\hline 2009 & 42 & 39 & 40 & 47 & 42 & 2009 & 29 & 34 & 42 & 36 & 35 \\
\hline 2010 & 47 & 44 & 48 & 42 & 45 & 2010 & 36 & 35 & 30 & 22 & 1 \\
\hline 2011 & 44 & 41 & 39 & 38 & 41 & 2011 & 27 & 28 & 26 & 26 & 27 \\
\hline 2012 & 36 & 47 & 44 & 38 & 41 & 2012 & 20 & 19 & 24 & 17 & 20 \\
\hline 2013 & 46 & 48 & 30 & 48 & 43 & 2013 & 25 & 23 & 20 & 16 & 21 \\
\hline 2014 & 37 & 39 & 37 & 38 & 38 & 2014 & 13 & 14 & 23 & 26 & 19 \\
\hline 2015 & 39 & 30 & 40 & 45 & 38 & 2015 & 24 & 26 & 15 & 16 & 20 \\
\hline 2016 & 40 & 37 & 34 & 37 & 37 & 2016 & 15 & 33 & 15 & 15 & 20 \\
\hline 2017 & 36 & 40 & 33 & 32 & 35 & 2017 & 18 & 20 & 15 & 20 & 18 \\
\hline 2018 & 24 & 32 & 36 & & & 2018 & 11 & 18 & 15 & & \\
\hline \multicolumn{6}{|c|}{$\begin{array}{c}\text { 177. Reasons for manufacturing loss-making } \\
\text { production: exploration of new markets, industry (\%) } \\
\text { 177. Причины производства убыточной продукции: } \\
\text { "завоевание" новых рынков сбыта, } \\
\text { промышленность (\%) }\end{array}$} & \multicolumn{6}{|c|}{$\begin{array}{c}\text { 178. Reasons for manufacturing loss-making } \\
\text { production: fluctuations of prices, industry (\%) } \\
\text { 178. Причины производства убыточной продукции: } \\
\text { непредвиденные колебания цен, } \\
\text { промышленность (\%) } \\
\end{array}$} \\
\hline 1995 & - & - & 14 & 9 & - & 1995 & - & - & 34 & 30 & - \\
\hline 1996 & 8 & 12 & 16 & 14 & 12 & 1996 & 33 & 30 & 29 & 21 & 28 \\
\hline 1997 & 10 & 19 & 17 & 23 & 17 & 1997 & 24 & 18 & 20 & 19 & 20 \\
\hline 1998 & 19 & 18 & 21 & 17 & 19 & 98 & 17 & 19 & 26 & 29 & 23 \\
\hline 1999 & 20 & 16 & 21 & 17 & 19 & 1999 & 32 & 28 & 26 & 27 & 28 \\
\hline 2000 & 21 & 16 & 18 & 1 & 18 & 2000 & 24 & 24 & 16 & 21 & 21 \\
\hline 2001 & 20 & 19 & 19 & 22 & $2 c$ & 2001 & 21 & 20 & 16 & 10 & 17 \\
\hline 2002 & 18 & 24 & 22 & 24 & 22 & 2002 & 16 & 18 & 14 & 17 & 16 \\
\hline 2003 & 27 & 24 & 20 & 22 & 23 & 2 & 2 & 17 & 15 & 19 & 18 \\
\hline 2004 & 19 & 22 & 22 & 23 & 22 & 2004 & 16 & 22 & 22 & 17 & 19 \\
\hline 2005 & 26 & 18 & 24 & 24 & 23 & 2005 & 1 & 1 & 23 & 20 & 19 \\
\hline 2006 & 26 & 28 & 30 & 3 & 25 & 2006 & 20 & 23 & 18 & 17 & 19 \\
\hline 2007 & 34 & 31 & 28 & 31 & 3 & 2007 & 17 & 1 & 24 & 21 & 19 \\
\hline 2008 & 23 & 20 & 21 & 26 & 23 & 2008 & 26 & 29 & 21 & 21 & 24 \\
\hline 2009 & 18 & 18 & 18 & 19 & 18 & 2009 & 33 & 20 & 29 & 22 & 26 \\
\hline 2010 & 24 & 23 & 25 & 2 & 24 & & 23 & 19 & 26 & 26 & 24 \\
\hline 2011 & 23 & 28 & 25 & 32 & 27 & 2011 & 30 & 25 & 22 & 26 & 26 \\
\hline 2012 & 24 & 27 & 30 & 27 & 27 & 2012 & 20 & 19 & 20 & 11 & 18 \\
\hline 2013 & 30 & 30 & 20 & 17 & 24 & 2013 & 14 & 10 & 9 & 13 & 12 \\
\hline 2014 & 30 & 23 & 26 & 17 & 24 & 2014 & 14 & 18 & 18 & 23 & 18 \\
\hline 2015 & 20 & 24 & 25 & 25 & 24 & 2015 & 31 & 28 & 29 & 18 & 26 \\
\hline 2016 & 19 & 18 & 15 & 15 & 17 & 2016 & 23 & 31 & 11 & 20 & 21 \\
\hline 2017 & 20 & 24 & 24 & 32 & 25 & 2017 & 13 & 11 & $19^{*}$ & 12 & 14 \\
\hline 2018 & 24 & 24 & 26 & & & 2018 & 13 & 21 & 15 & & \\
\hline
\end{tabular}




\begin{tabular}{|c|c|c|c|c|c|c|c|c|c|c|c|}
\hline Year & $I Q$ & II Q & III Q & IV Q & Annual & Year & $\overline{I Q}$ & II Q & III Q & $I V Q$ & Annual \\
\hline \multicolumn{6}{|c|}{$\begin{array}{r}\text { 179. Reasons for manufacturing loss-making } \\
\text { production: new and perhaps temporarily loss-making } \\
\text { production, industry (\%) } \\
\text { 179. Причины производства убыточной продукции: } \\
\text { новая и, возможно, временно } \\
\text { убыточная продукция, промышленность (\%) }\end{array}$} & \multicolumn{2}{|c|}{$\begin{array}{l}\text { 121. Доля предприятий, у которых за год } \\
\text { производственные мощности } \\
\text { увеличились, промышленность (\%) }\end{array}$} & & & & \\
\hline 1992 & - & - & - & - & - & 1992 & - & - & 13 & 15 & - \\
\hline 1993 & - & - & - & - & - & 1993 & 17 & 20 & 20 & 17 & 18 \\
\hline 1994 & - & - & - & - & - & 994 & 14 & 16 & 19 & 13 & 16 \\
\hline 1995 & - & - & 18 & 14 & - & 1995 & 17 & 14 & 19 & 14 & 16 \\
\hline 1996 & 14 & 14 & 18 & 15 & 15 & 1996 & 16 & 14 & 9 & 11 & 12 \\
\hline 1997 & 15 & 17 & 18 & 18 & 17 & 997 & 8 & 10 & 11 & 19 & 12 \\
\hline 1998 & 14 & 16 & 15 & 19 & 16 & 998 & 17 & 12 & 15 & 19 & 16 \\
\hline 1999 & 17 & 17 & 18 & 18 & 17 & 1999 & 22 & 22 & 32 & 31 & 27 \\
\hline 2000 & 19 & 18 & 17 & 22 & 19 & 2000 & 31 & 28 & 35 & 34 & 32 \\
\hline 2001 & 22 & 19 & 20 & 18 & 20 & 2001 & 32 & 32 & 33 & 26 & 31 \\
\hline 2002 & 25 & 20 & 21 & 22 & 22 & 2002 & 30 & 30 & 24 & 30 & 28 \\
\hline 2003 & 18 & 24 & 20 & 25 & 22 & 2003 & 31 & 26 & 29 & 34 & 30 \\
\hline 2004 & 18 & 16 & 21 & 19 & 19 & 2004 & 33 & 38 & 37 & 37 & 36 \\
\hline 2005 & 20 & 15 & 19 & 22 & 19 & 2005 & 32 & 29 & 40 & 33 & 34 \\
\hline 2006 & 18 & 26 & 20 & 16 & 20 & 2006 & 34 & 32 & 45 & 45 & 39 \\
\hline 2007 & 23 & 25 & 18 & 18 & 21 & 2007 & 41 & 43 & 47 & 39 & 42 \\
\hline 2008 & 21 & 17 & 21 & 19 & 20 & 2008 & 41 & 43 & 44 & 31 & 40 \\
\hline 2009 & 13 & 16 & 16 & 14 & 15 & 2009 & 24 & 23 & 24 & 21 & 23 \\
\hline 2010 & 17 & 25 & 16 & 17 & 19 & 10 & 28 & 31 & 35 & 41 & 34 \\
\hline & 22 & 21 & 23 & 24 & 22 & & 34 & 35 & 48 & 37 & 38 \\
\hline 2012 & 24 & 26 & 23 & 23 & 24 & 2012 & 41 & 43 & 46 & 34 & 41 \\
\hline 2013 & 25 & 21 & 20 & 19 & 21 & 2013 & 45 & 29 & 40 & 39 & 38 \\
\hline 2014 & 14 & 14 & 23 & 17 & 17 & & 44 & 31 & 39 & 38 & 38 \\
\hline 2015 & 19 & 20 & 10 & 18 & 17 & 2015 & 37 & 35 & 33 & 35 & 35 \\
\hline & 17 & 12 & 11 & 17 & 14 & & 31 & 37 & 28 & 38 & 34 \\
\hline 2017 & 13 & 22 & $14^{\star}$ & 7 & 14 & 2017 & 32 & 29 & $35^{*}$ & 44 & 35 \\
\hline 2018 & 16 & 26 & 10 & & & 2018 & 45 & 27 & 28 & & \\
\hline \multicolumn{6}{|c|}{$\begin{array}{l}\text { 122. Share of enterprises with unchanged production } \\
\text { capacities in the last } 12 \text { months, industry (\%) } \\
\text { 122. Доля предприятий, у которых за год } \\
\text { производственные мощности не изменились, } \\
\text { промышленность (\%) }\end{array}$} & \multicolumn{6}{|c|}{$\begin{array}{l}\text { 123. Share of enterprises with decreased production } \\
\text { capacities in the last } 12 \text { months, industry (\%) } \\
\text { 123. Доля предприятий, у которых за год } \\
\text { производственные мощности уменьшились, } \\
\text { промышленность (\%) } \\
\end{array}$} \\
\hline 1992 & - & - & 20 & 26 & - & 1992 & - & - & 67 & 60 & - \\
\hline & 24 & 22 & 2 & ? & 24 & & 60 & 58 & 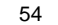 & 60 & 58 \\
\hline & 26 & 22 & 22 & 20 & 22 & & 6 & 6 & 5 & 67 & 62 \\
\hline & 21 & 28 & 29 & 27 & 26 & & 6 & 5 & 5 & 60 & 58 \\
\hline & 23 & 22 & 27 & 24 & 24 & & 6 & 6 & 64 & 65 & 63 \\
\hline & 21 & 31 & 31 & 28 & 28 & & 7 & 5 & 5 & 54 & 60 \\
\hline & 31 & 31 & 32 & 27 & 30 & & 5 & 5 & 53 & 54 & 54 \\
\hline & 34 & 36 & 34 & 35 & 35 & & 44 & 4 & 34 & 35 & 39 \\
\hline & 36 & 38 & 3 & 34 & 3 & & & & 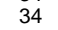 & 3 & 33 \\
\hline & 35 & 34 & 29 & 37 & 34 & & 3 & 3 & 38 & 37 & 35 \\
\hline & 39 & 38 & 40 & & 39 & & 3 & 3 & 37 & 32 & 33 \\
\hline & 35 & 37 & ? & & 3 & & & & & 3 & 34 \\
\hline & 31 & 3 & 3 & 3 & 36 & & 3 & & 2 & 24 & 28 \\
\hline & 33 & 36 & 3 & 3 & 35 & & 3 & 3 & 2 & 30 & 31 \\
\hline & 41 & 35 & 3 & & 3 & & 2 & & 2 & 2 & 25 \\
\hline & 35 & 32 & 2 & 4 & 34 & & 2 & & 2 & 21 & 24 \\
\hline & 36 & 30 & 3 & 1 & 34 & & . & 2 & 22 & 36 & 27 \\
\hline & 39 & 36 & 2 & & 3 & & 3 & & 4 & 42 & 42 \\
\hline 20 & 43 & 41 & 37 & 3 & 38 & 20 & 2 & & 2 & 27 & 28 \\
\hline 20 & 31 & 40 & 27 & 3 & 3 & & 3 & 2 & 2 & 30 & 29 \\
\hline & 32 & 32 & 38 & 37 & 35 & & 2 & 2 & 1 & 29 & 24 \\
\hline & 29 & 32 & 37 & 26 & 31 & & 2 & 3 & 2 & 35 & 31 \\
\hline & 33 & 33 & 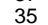 & & 32 & & & 3 & 2 & & 30 \\
\hline & 35 & 35 & 39 & 37 & 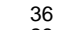 & & 2 & 3 & 2 & 2 & 29 \\
\hline 201 & 33 & 24 & 22 & 33 & 28 & 2016 & 37 & 39 & 50 & 29 & 39 \\
\hline 2017 & 30 & 29 & 34 & 33 & 31 & 2017 & 38 & 43 & $33^{*}$ & 23 & 34 \\
\hline 2018 & 24 & 24 & 25 & & & 2018 & 32 & 48 & 47 & & \\
\hline
\end{tabular}




\begin{tabular}{|c|c|c|c|c|c|c|c|c|c|c|c|}
\hline$\frac{\text { Year }}{124 . \text { Sh }}$ & $\mathrm{IQ}$ & $\mathrm{IIQ}$ & III Q & IV Q & Annual & Year & $\mathrm{IQ}$ & $\| \mathrm{Q}$ & III Q & $\mathrm{IVQ}$ & Annual \\
\hline $\begin{array}{l}\text { of the last } 6 \text { months and anticipating no investments in } \\
\text { the next } 6 \text { months, industry (\%) } \\
\text { 124. Доля предприятий, у которых капитальных } \\
\text { вложений не было в предшествующие } 6 \text { месяцев и } \\
\text { не ожидается в следующие } 6 \text { месяцев, } \\
\text { промышленность (\%) }\end{array}$ & \multicolumn{5}{|c|}{$\begin{array}{l}\text { of enterprises with zero capital investments } \\
\text { months and anticipating no investments in } \\
\text { the next } 6 \text { months, industry (\%) }\end{array}$} & \multicolumn{2}{|c|}{$\begin{array}{c}\text { 125. Степень реализации инвестиционных планов } \\
\text { последних } 6 \text { месяцев, промышленность, }(\%, \\
\text { полная реализация = } 100 \%)\end{array}$} & & & & \\
\hline $\begin{array}{l}1992 \\
1993 \\
1994 \\
1995 \\
1996 \\
1997 \\
1998 \\
1999 \\
2000 \\
2001 \\
2002 \\
2003 \\
2004 \\
2005 \\
2006 \\
2007 \\
2008 \\
2009 \\
2010 \\
2011 \\
2012 \\
2013 \\
2014 \\
2015 \\
2016 \\
2017 \\
2018\end{array}$ & \begin{tabular}{|c|}
0 \\
9 \\
21 \\
21 \\
35 \\
36 \\
41 \\
49 \\
38 \\
28 \\
30 \\
26 \\
24 \\
24 \\
25 \\
18 \\
15 \\
16 \\
16 \\
28 \\
28 \\
37 \\
34 \\
24 \\
29 \\
32 \\
26 \\
30 \\
26
\end{tabular} & $\begin{array}{l}19 \\
27 \\
34 \\
30 \\
33 \\
41 \\
46 \\
38 \\
30 \\
22 \\
27 \\
26 \\
-{ }^{21} \\
- \\
- \\
- \\
- \\
- \\
- \\
- \\
- \\
- \\
- \\
- \\
- \\
-\end{array}$ & $\begin{array}{c}13 \\
7 \\
33 \\
28 \\
38 \\
42 \\
51 \\
30 \\
31 \\
23 \\
19 \\
28 \\
25 \\
18 \\
9 \\
15 \\
20 \\
31 \\
28 \\
25 \\
16 \\
18 \\
30 \\
31 \\
38 \\
26\end{array}$ & $\begin{array}{l}18 \\
18 \\
21 \\
36 \\
39 \\
41 \\
42 \\
31 \\
26 \\
19 \\
19 \\
20 \\
- \\
- \\
- \\
- \\
- \\
- \\
- \\
- \\
- \\
- \\
- \\
- \\
- \\
-\end{array}$ & $\begin{array}{l}12 \\
15 \\
27 \\
32 \\
36 \\
41 \\
47 \\
34 \\
29 \\
24 \\
23 \\
24 \\
25 \\
18 \\
12 \\
15 \\
18 \\
29 \\
32 \\
30 \\
20 \\
23 \\
31 \\
29 \\
34 \\
26\end{array}$ & $\begin{array}{l}1992 \\
1993 \\
1994 \\
1995 \\
1996 \\
1997 \\
1998 \\
1999 \\
2000 \\
2001 \\
2002 \\
2003 \\
2004 \\
2005 \\
2006 \\
2007 \\
2008 \\
2009 \\
2010 \\
2011 \\
2012 \\
2013 \\
2014 \\
2015 \\
2016 \\
2017 \\
2018\end{array}$ & $\begin{array}{l}- \\
53 \\
47 \\
40 \\
37 \\
51 \\
48 \\
48 \\
55 \\
63 \\
65 \\
68 \\
71 \\
71 \\
74 \\
76 \\
75 \\
65 \\
65 \\
66 \\
73 \\
74 \\
75 \\
70 \\
74 \\
78 \\
76\end{array}$ & $\begin{array}{l}58 \\
54 \\
37 \\
36 \\
35 \\
51 \\
47 \\
53 \\
57 \\
58 \\
60 \\
64 \\
68 \\
73 \\
75 \\
68 \\
71 \\
59 \\
66 \\
69 \\
75 \\
75 \\
68 \\
62 \\
67 \\
71 \\
67\end{array}$ & $\begin{array}{l}54 \\
53 \\
41 \\
36 \\
43 \\
45 \\
39 \\
55 \\
63 \\
57 \\
60 \\
66 \\
68 \\
68 \\
77 \\
67 \\
70 \\
58 \\
66 \\
68 \\
76 \\
78 \\
67 \\
71 \\
68 \\
77 \\
72\end{array}$ & $\begin{array}{l}55 \\
48 \\
46 \\
45 \\
48 \\
56 \\
47 \\
61 \\
65 \\
64 \\
70 \\
73 \\
76 \\
71 \\
84 \\
80 \\
75 \\
65 \\
72 \\
73 \\
75 \\
79 \\
74 \\
74 \\
79 \\
80\end{array}$ & $\begin{array}{l}- \\
52 \\
43 \\
39 \\
41 \\
51 \\
45 \\
54 \\
60 \\
61 \\
64 \\
68 \\
71 \\
71 \\
77 \\
73 \\
73 \\
62 \\
67 \\
69 \\
75 \\
76 \\
71 \\
69 \\
72 \\
76\end{array}$ \\
\hline \multicolumn{6}{|c|}{$\begin{array}{l}\text { 126. Share of enterprises with no investment plans in } \\
\text { the last } 6 \text { months, industry (\%) } \\
\text { 126. Доля предприятий, у которых в последние } 6 \\
\text { месяцев инвестиционных планов не было, } \\
\text { промышленность (\%) }\end{array}$} & \multicolumn{6}{|c|}{$\begin{array}{c}\text { 127. Marginal (affordable) interest rate for fixed } \\
\text { investment (on a rouble loan for 2-3 years loan } \\
\text { period), industry (\%) } \\
\text { 127. Предельная (допустимая) процентная ставка } \\
\text { для капитальных вложений (по рублевой ссуде на } \\
2-3 \text { года), промышленность (\%) }\end{array}$} \\
\hline $\begin{array}{l}1996 \\
1997 \\
1998 \\
1999 \\
2000 \\
2001 \\
2002 \\
2003 \\
2004 \\
2005 \\
2006 \\
2007 \\
2008 \\
2009 \\
2010 \\
2011 \\
2012 \\
2013 \\
2014 \\
2015 \\
2016 \\
2017 \\
2018\end{array}$ & $\begin{array}{l}- \\
39 \\
34 \\
33 \\
29 \\
25 \\
22 \\
23 \\
14 \\
19 \\
18 \\
16 \\
17 \\
26 \\
29 \\
30 \\
30 \\
26 \\
27 \\
20 \\
30 \\
31 \\
29\end{array}$ & $\begin{array}{l}- \\
49 \\
46 \\
35 \\
33 \\
25 \\
22 \\
27 \\
22 \\
17 \\
20 \\
16 \\
19 \\
40 \\
40 \\
31 \\
31 \\
31 \\
33 \\
28 \\
29 \\
36 \\
26\end{array}$ & $\begin{array}{c}35 \\
36 \\
33 \\
28 \\
29 \\
22 \\
18 \\
21 \\
17 \\
9 \\
14 \\
17 \\
16 \\
38 \\
33 \\
28 \\
19 \\
18 \\
19 \\
19 \\
23 \\
21^{*} \\
28\end{array}$ & $\begin{array}{l}42 \\
42 \\
43 \\
37 \\
26 \\
22 \\
14 \\
14 \\
18 \\
12 \\
17 \\
19 \\
30 \\
33 \\
34 \\
33 \\
39 \\
28 \\
34 \\
29 \\
22 \\
22\end{array}$ & $\begin{array}{l}- \\
42 \\
39 \\
33 \\
29 \\
24 \\
19 \\
21 \\
18 \\
14 \\
17 \\
17 \\
20 \\
34 \\
34 \\
31 \\
30 \\
26 \\
28 \\
24 \\
26 \\
27\end{array}$ & $\begin{array}{l}1996 \\
1997 \\
1998 \\
1999 \\
2000 \\
2001 \\
2002 \\
2003 \\
2004 \\
2005 \\
2006 \\
2007 \\
2008 \\
2009 \\
2010 \\
2011 \\
2012 \\
2013 \\
2014 \\
2015 \\
2016 \\
2017 \\
2018\end{array}$ & $\begin{array}{c}10.6 \\
8.2 \\
10.8 \\
10.6 \\
9.6 \\
9.2 \\
9.2 \\
7.4 \\
8.6 \\
6.8 \\
7.2 \\
7.2 \\
8.6 \\
8.2 \\
7.4 \\
6.8 \\
7.0 \\
8.0 \\
9.0 \\
8.0 \\
7.1 \\
6.2\end{array}$ & $\begin{array}{c}15.2 \\
8.0 \\
9.2 \\
10.8 \\
10.6 \\
9.8 \\
9.0 \\
9.4 \\
8.2 \\
7.6 \\
6.8 \\
7.4 \\
7.8 \\
8.4 \\
7.8 \\
7.4 \\
8.2 \\
8.0 \\
7.8 \\
8.6 \\
7.8 \\
7.3 \\
5.9\end{array}$ & $\begin{array}{c}17.6 \\
8.4 \\
9.8 \\
10.4 \\
9.0 \\
9.0 \\
8.6 \\
9.6 \\
7.6 \\
7.4 \\
7.2 \\
7.8 \\
8.0 \\
8.2 \\
7.6 \\
7.4 \\
8.0 \\
7.6 \\
7.6 \\
8.6 \\
7.4 \\
6.1^{\star} \\
6.0\end{array}$ & $\begin{array}{l}12.2 \\
8.4 \\
10.2 \\
10.6 \\
9.4 \\
9.2 \\
8.8 \\
8.0 \\
7.6 \\
8.0 \\
6.8 \\
6.8 \\
8.4 \\
7.8 \\
6.8 \\
7.0 \\
7.2 \\
8.2 \\
7.8 \\
9.2 \\
7.8 \\
6.3\end{array}$ & \begin{tabular}{|c|}
- \\
8.8 \\
9.4 \\
10.6 \\
10.0 \\
9.4 \\
8.9 \\
9.0 \\
7.7 \\
7.9 \\
6.9 \\
7.3 \\
7.8 \\
8.2 \\
7.6 \\
7.3 \\
7.5 \\
7.6 \\
7.8 \\
8.8 \\
7.8 \\
6.7
\end{tabular} \\
\hline
\end{tabular}

2) Since 2004 REB has been measuring this index twice a year. 


\begin{tabular}{|c|c|c|c|c|c|c|c|c|c|c|c|}
\hline Year & $\mathrm{IQ}$ & $\mathrm{IIQ}$ & $\mathrm{IIIQ}$ & $\mathrm{IVQ}$ & Annual & Year & $\overline{I Q}$ & $\| \mathrm{II}$ & III Q & $\mathrm{IVQ}$ & Annual \\
\hline \multicolumn{6}{|c|}{$\begin{array}{l}\text { 128. Competition on the main product markets (a five- } \\
\text { grade scale: } 1 \text { - absent, } 5 \text { - menace of ousting from } \\
\text { the market), industry } \\
\text { 128. Острота конкуренции на основных рынках } \\
\text { сбыта (5-балльная шкала: } 1 \text { - отсутствует, } 5 \text { - на } \\
\text { грани вытеснения с рынка), промышленность }\end{array}$} & \multicolumn{6}{|c|}{$\begin{array}{l}\text { 129. Competition with foreign makers on the main } \\
\text { product markets (a five-grade scale: } 1 \text { - absent, } 5 \text { - } \\
\text { menace of ousting from the market), industry } \\
\text { 129. Острота конкуренции на основных рынках } \\
\text { сбыта со стороны нероссийских производителей } \\
\text { (5-балльная шкала: } 1 \text { - отсутствует, } 5 \text { - на грани } \\
\text { вытеснения с рынка), промышленность }\end{array}$} \\
\hline 1995 & - & 3.1 & - & 3.1 & 3.1 & 1995 & - & 2.3 & - & 2.5 & 2.4 \\
\hline 1996 & - & 3.3 & - & 3.5 & 3.4 & 1996 & - & 2.5 & - & 2.8 & 2.65 \\
\hline 1997 & - & 3.4 & - & 3.4 & 3.4 & 1997 & - & 2.8 & - & 2.9 & 2.85 \\
\hline 1998 & - & 3.7 & - & 3.5 & 3.6 & 1998 & - & 3.1 & - & 2.8 & 2.95 \\
\hline 1999 & - & 3.3 & - & 3.4 & 3.35 & 99 & - & 2.8 & - & 2.3 & 2.55 \\
\hline 2000 & - & 3.5 & - & 3.5 & 3.5 & 2000 & - & 2.6 & - & 2.8 & 2.7 \\
\hline 2001 & - & 3.5 & - & 3.7 & 3.6 & 2001 & - & 2.6 & - & 2.8 & 2.7 \\
\hline 2002 & - & 3.6 & - & 3.7 & 3.65 & 2002 & - & 2.6 & - & 2.6 & 2.6 \\
\hline 2003 & - & 3.6 & - & 3.7 & 3.65 & 2003 & - & 2.9 & - & 2.9 & 2.9 \\
\hline 2004 & - & 3.6 & - & 3.6 & 36 & 2004 & - & 2.9 & - & 2.8 & 2.85 \\
\hline 2005 & - & 3.6 & - & 3.6 & 3.6 & 2005 & - & 3.3 & - & 3.0 & .15 \\
\hline 2006 & - & 3.7 & - & 3.7 & 3.7 & 2006 & - & 2.8 & - & 2.9 & 2.85 \\
\hline 2007 & - & 3.5 & - & 3.5 & 3.5 & & - & 3.0 & - & 3.0 & 3.0 \\
\hline 2008 & - & 3.5 & - & 3.6 & 3.5 & 8 & - & 3.1 & - & 2.7 & 2.9 \\
\hline 2009 & - & 3.9 & - & 3.5 & 3. & 9 & - & 3.5 & - & 3.2 & 3.35 \\
\hline 2010 & - & 3.7 & - & 3.7 & 3.7 & & - & 3.1 & - & 3.1 & 3.1 \\
\hline 2011 & - & 3.4 & - & 3.3 & & & - & 3.0 & - & 3.2 & 3.1 \\
\hline 20 & - & 3.7 & - & 3.8 & & & - & 3.3 & - & 3.5 & 3.4 \\
\hline 2013 & - & 3.7 & - & 3.6 & 3. & 2013 & - & 3.4 & - & 3.3 & 3.35 \\
\hline 201 & - & 3.7 & - & 3.4 & & & - & 3.3 & - & 3.2 & 3.25 \\
\hline 2015 & - & 3.6 & - & 3.7 & & & - & 3.3 & - & 3.1 & 3.2 \\
\hline 2016 & - & 3.6 & - & 3.9 & & & - & 3.0 & - & 3.1 & 3.05 \\
\hline 2017 & - & 3.9 & - & 3.8 & 3.8 & 2017 & - & 3.0 & - & 3.0 & 3.0 \\
\hline 2018 & - & 3.5 & & & & 2018 & - & 3.0 & & & \\
\hline \multicolumn{6}{|c|}{$\begin{array}{l}\text { 101. Limitations to production: insufficient demand, } \\
\text { present situation, agriculture (share of enterprises, \%) }\end{array}$} & \multicolumn{6}{|c|}{$\begin{array}{l}\text { 102. Limitations to production: shortage of raw } \\
\text { material and semi-finished products, present } \\
\text { situation, agriculture (share of enterprises, \%) }\end{array}$} \\
\hline \multicolumn{6}{|c|}{$\begin{array}{l}\text { 101. Ограничения производства: недостаток } \\
\text { спроса, текущая ситуация, сельское хозяйство } \\
\text { (доля предприятий, \%) }\end{array}$} & \multicolumn{6}{|c|}{$\begin{array}{l}\text { 102. Ограничения производства: нехватка сырья, } \\
\text { материалов, полуфабрикатов, текущая ситуация, } \\
\text { сельское хозяйство (доля предприятий, \%) }\end{array}$} \\
\hline 1992 & 11 & 18 & 24 & 24 & 19 & 1992 & 36 & 22 & 18 & 12 & 22 \\
\hline 1993 & 16 & 28 & 38 & 20 & 28 & & 29 & 19 & 16 & 9 & 18 \\
\hline 1994 & 3 & 47 & 49 & & & & 13 & 3 & 6 & 12 & 8 \\
\hline & 3 & 33 & 37 & 37 & 35 & & 13 & 3 & 10 & 14 & 10 \\
\hline & 38 & 33 & 46 & 43 & 40 & 3 & 10 & 7 & 2 & 4 & 6 \\
\hline 19 & 25 & 32 & 37 & 33 & & & 5 & 6 & 0 & 2 & 3 \\
\hline & 33 & 40 & 26 & 14 & 25 & & 2 & 5 & 4 & 16 & 7 \\
\hline & 21 & 10 & 24 & 2 & $1 s$ & & 19 & 25 & 3 & 12 & 15 \\
\hline & 9 & 24 & 18 & 1 & 1 & & 18 & 7 & 7 & 2 & 8 \\
\hline 20 & 3 & 13 & 11 & 25 & 1 & & 3 & 10 & 9 & 7 & 7 \\
\hline & 15 & 15 & 42 & 0 & & & 15 & 3 & 0 & 0 & 5 \\
\hline & 28 & 17 & 31 & 16 & 2 & & 3 & 10 & 0 & 16 & 7 \\
\hline 2004 & 9 & 19 & 20 & 17 & 16 & 20 & 0 & 10 & 3 & 6 & 5 \\
\hline & 21 & 2 & 2 & & & & 7 & 4 & 0 & 6 & 4 \\
\hline 20 & 32 & 42 & 2 & 19 & 25 & & 5 & 8 & 9 & 8 & \\
\hline & 25 & 31 & 18 & 6 & 2 & & 6 & 0 & 9 & 0 & 4 \\
\hline & 4 & 20 & 29 & & & & 8 & 0 & 6 & 5 & 5 \\
\hline & 36 & 30 & 44 & 44 & $3 \varepsilon$ & & 12 & 11 & 0 & 4 & 7 \\
\hline 20 & 22 & 52 & 29 & 14 & 0 & & 0 & 0 & 10 & 18 & 7 \\
\hline & 16 & 16 & 30 & & & & 16 & 8 & 5 & 10 & 10 \\
\hline & 32 & 22 & 30 & 21 & 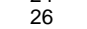 & & 5 & 6 & 0 & 0 & 3 \\
\hline & 15 & 5 & 38 & 38 & 24 & & 10 & 6 & 0 & 0 & 4 \\
\hline & 20 & 7 & 16 & 11 & & & 10 & 0 & 5 & 0 & 4 \\
\hline & 10 & 25 & 33 & 16 & 21 & 20 & 5 & 5 & 0 & 0 & 3 \\
\hline 20 & 15 & 16 & 19 & 30 & 2 & 20 & 8 & 11 & 0 & 0 & 5 \\
\hline & 31 & 24 & 33 & 21 & 27 & 2017 & 6 & 0 & 5 & 0 & 3 \\
\hline 201 & 45 & 39 & & & & 2018 & 0 & 6 & & & \\
\hline
\end{tabular}




\begin{tabular}{|c|c|c|c|c|c|c|c|c|c|c|c|}
\hline Year & $\mathrm{IQ}$ & II Q & III Q & IV Q & Annual & Year & $I Q$ & $\| \mathrm{Q}$ & III Q & $\mathrm{IVQ}$ & Annual \\
\hline \multicolumn{6}{|c|}{$\begin{array}{l}\text { 103. Limitations to production: shortage of financial } \\
\text { resources, present situation, agriculture (share of } \\
\text { enterprises, \%) } \\
\text { 103. Ограничения производства: нехватка } \\
\text { финансовых ресурсов, текущая ситуация, сельское } \\
\text { хозяйство (доля предприятий, \%) }\end{array}$} & \multicolumn{6}{|c|}{$\begin{array}{c}\text { 104. Limitations to production: shortage of labour, } \\
\text { present situation, agriculture (share of enterprises, } \\
\% \text { ) } \\
\text { 104. Ограничения производства: нехватка } \\
\text { рабочей силы, текущая ситуация, сельское } \\
\text { хозяйство (доля предприятий, \%) }\end{array}$} \\
\hline 1992 & 61 & 68 & 45 & 61 & 59 & 1992 & 11 & 6 & 8 & 6 & 8 \\
\hline 1993 & 73 & 68 & 68 & 74 & 71 & 1993 & 4 & 8 & 12 & 5 & 7 \\
\hline 1994 & 72 & 80 & 68 & 77 & 74 & 1994 & 2 & 3 & 2 & 0 & 2 \\
\hline 1995 & 79 & 64 & 67 & 72 & 70 & 1995 & 8 & 3 & 4 & 2 & 4 \\
\hline 1996 & 84 & 74 & 71 & 74 & 76 & 1996 & 0 & 2 & 0 & 0 & 0 \\
\hline 1997 & 75 & 76 & 73 & 72 & 74 & 1997 & 0 & 0 & 0 & 4 & 1 \\
\hline 1998 & 69 & 83 & 85 & 68 & 76 & 1998 & 3 & 0 & 0 & 1 & 1 \\
\hline 1999 & 77 & 67 & 70 & 78 & 73 & 1999 & 4 & 4 & 3 & 2 & 3 \\
\hline 2000 & 82 & 76 & 82 & 77 & 79 & 2000 & 3 & 2 & 11 & 9 & 6 \\
\hline 2001 & 77 & 55 & 86 & 65 & 71 & 2001 & 10 & 8 & 11 & 15 & 11 \\
\hline 2002 & 77 & 73 & 48 & 100 & 74 & 2002 & 8 & 12 & 21 & 14 & 14 \\
\hline 2003 & 72 & 66 & 75 & 47 & 65 & 2003 & 3 & 10 & 19 & 5 & 9 \\
\hline 2004 & 63 & 48 & 63 & 49 & 56 & 2004 & 11 & 10 & 14 & 6 & 10 \\
\hline 2005 & 63 & 57 & 72 & 60 & 63 & 2005 & 12 & 14 & 14 & 9 & 12 \\
\hline 2006 & 68 & 67 & 61 & 50 & 62 & 2006 & 16 & 8 & 17 & 8 & 12 \\
\hline 2007 & 63 & 62 & 82 & 47 & 63 & 2007 & 19 & 15 & 32 & 18 & 21 \\
\hline 2008 & 71 & 65 & 76 & 60 & 68 & 2008 & 29 & 25 & 12 & 10 & 19 \\
\hline 2009 & 52 & 56 & 52 & 60 & 55 & 2009 & 8 & 15 & 4 & 4 & 8 \\
\hline 2010 & 56 & 48 & 62 & 64 & 57 & 2010 & 11 & 9 & 10 & 9 & 10 \\
\hline 2011 & 47 & 56 & 55 & 38 & 49 & 2011 & 5 & 12 & 10 & 10 & 9 \\
\hline 2012 & 64 & 44 & 57 & 38 & 51 & 2012 & 23 & 17 & 17 & 17 & 18 \\
\hline 2013 & 30 & 56 & 54 & 63 & 51 & 2013 & 20 & 31 & 15 & 19 & 21 \\
\hline 2014 & 60 & 47 & 47 & 58 & 53 & 2015 & 10 & 7 & 21 & 21 & 15 \\
\hline 2015 & 70 & 50 & 56 & 42 & 54 & 2015 & 10 & 15 & 17 & 5 & 12 \\
\hline 2016 & 54 & 58 & 69 & 55 & 59 & 2016 & 8 & 5 & 19 & 15 & 12 \\
\hline 2017 & 44 & 59 & 43 & 53 & 50 & 2017 & 19 & 18 & 14 & 21 & 18 \\
\hline 2018 & 27 & 56 & & & & 2018 & 9 & 22 & & & \\
\hline
\end{tabular}




\begin{tabular}{|c|c|c|c|c|c|c|c|c|c|c|c|}
\hline Year & IQ & II Q & $\mathrm{IIIQ}$ & $I V Q$ & Annual & Year & $\mathrm{IQ}$ & IIQ & $\mathrm{III} \mathrm{Q}$ & IVQ & Annual \\
\hline \multicolumn{6}{|c|}{$\begin{array}{l}\text { 105. Limitations to production: shortage of equipment } \\
\text { and premises, present situation, agriculture (share of } \\
\text { enterprises, \%) } \\
\text { 105. Ограничения производства: нехватка } \\
\text { оборудования, помещений, текущая ситуация, } \\
\text { сельское хозяйство (доля предприятий, \%) }\end{array}$} & \multicolumn{6}{|c|}{$\begin{array}{l}\text { 106. Limitations to production: high prices for raw } \\
\text { materials, semi-finished goods, present situation, } \\
\text { agriculture (enterprise share, \%) } \\
\text { 106. Ограничения производства: высокие цены на } \\
\text { сырьё, материалы, полуфабрикаты, текущая } \\
\text { ситуация, сельское хозяйство (доля предприятий, } \\
\text { \%) }\end{array}$} \\
\hline 1992 & 11 & 10 & 11 & 12 & 11 & 1992 & - & - & - & - & - \\
\hline 1993 & 4 & 9 & 16 & 20 & 12 & 1993 & 69 & 62 & 58 & 55 & 61 \\
\hline 1994 & 11 & 8 & 10 & 7 & 9 & 1994 & 62 & 50 & 56 & 66 & 59 \\
\hline 1995 & 10 & 2 & 4 & 2 & 4 & 1995 & 50 & 49 & 61 & 56 & 54 \\
\hline 1996 & 0 & 7 & 2 & 6 & 4 & 1996 & 52 & 47 & 44 & 48 & 48 \\
\hline 1997 & 8 & 4 & 10 & 9 & 8 & 1997 & 54 & 50 & 41 & 48 & 48 \\
\hline 1998 & 7 & 10 & 13 & 9 & 10 & 1998 & 40 & 45 & 40 & 49 & 44 \\
\hline 1999 & 6 & 6 & 12 & 10 & 8 & 1999 & 43 & 57 & 67 & 49 & 54 \\
\hline 2000 & 6 & 2 & 4 & 9 & 5 & 2000 & 68 & 48 & 64 & 64 & 61 \\
\hline 2001 & 7 & 10 & 0 & 9 & 6 & 2001 & 43 & 60 & 51 & 49 & 51 \\
\hline 2002 & 0 & 15 & 3 & 29 & 12 & 2002 & 54 & 52 & 67 & 57 & 58 \\
\hline 2003 & 3 & 3 & 6 & 5 & 4 & 2003 & 56 & 45 & 56 & 47 & 51 \\
\hline 2004 & 6 & 6 & 9 & 14 & 9 & 2004 & 69 & 61 & 74 & 60 & 66 \\
\hline 2005 & 7 & 18 & 17 & 14 & 14 & 2005 & 65 & 29 & 52 & 54 & 50 \\
\hline 2006 & 5 & 0 & 4 & 15 & 6 & 2006 & 47 & 75 & 70 & 54 & 61 \\
\hline 2007 & 19 & 8 & 36 & 35 & 24 & 2007 & 50 & 38 & 45 & 88 & 55 \\
\hline 2008 & 4 & 10 & 12 & 20 & 12 & 2008 & 46 & 55 & 59 & 45 & 51 \\
\hline 2009 & 12 & 11 & 12 & 4 & 10 & 2009 & 56 & 44 & 44 & 50 & 49 \\
\hline 2010 & 22 & 26 & 5 & 9 & 15 & 2010 & 11 & 52 & 43 & 55 & 40 \\
\hline 2011 & 0 & 16 & 5 & 14 & 9 & 2011 & 58 & 32 & 40 & 48 & 44 \\
\hline 2012 & 14 & 11 & 13 & 25 & 16 & 2012 & 50 & 28 & 43 & 33 & 39 \\
\hline 2013 & 20 & 19 & 8 & 6 & 13 & 2013 & 40 & 38 & 54 & 63 & 49 \\
\hline 2014 & 20 & 20 & 11 & 21 & 18 & 2014 & 40 & 53 & 42 & 32 & 42 \\
\hline 2015 & 25 & 20 & 11 & 16 & 18 & 2015 & 30 & 45 & 50 & 47 & 43 \\
\hline 2016 & 8 & 21 & 6 & 15 & 13 & 2016 & 69 & 37 & 31 & 15 & 38 \\
\hline 2017 & 6 & 6 & 5 & 0 & 4 & 2017 & 38 & 41 & 24 & 32 & 34 \\
\hline 2018 & 0 & 0 & & & & 2018 & 55 & 22 & & & \\
\hline \multirow{3}{*}{\multicolumn{6}{|c|}{$\begin{array}{c}\text { 160. Share of enterprise directors comprehending } \\
\text { government's economic policy of last 3-4 months, } \\
\text { agriculture, \%) } \\
\text { 160. Доля руководителей, понимающих } \\
\text { экономическую политику правительства за } \\
\text { последние } 3-4 \text { месяца, сельское хозяйство (\%) }\end{array}$}} & \multirow{3}{*}{\multicolumn{6}{|c|}{$\begin{array}{l}\text { 161. Share of enterprise directors not comprehending } \\
\text { government's economic policy of last 3-4 months, } \\
\text { agriculture, \%) } \\
\text { 161. Доля руководителей, не понимающих } \\
\text { экономическую политику правительства за } \\
\text { последние 3-4 месяца, сельское хозяйство (\%) }\end{array}$}} \\
\hline & & & & & & & & & & & \\
\hline & & & & & & & & & & & \\
\hline 1993 & 27 & 34 & 17 & 28 & 26 & 1993 & 52 & 46 & 62 & 42 & 50 \\
\hline & 18 & 21 & 17 & 14 & 18 & 1994 & 59 & 57 & 61 & 61 & 59 \\
\hline 1995 & 6 & - & 12 & 16 & 11 & 1995 & 77 & - & 75 & 63 & 72 \\
\hline 1996 & 21 & 17 & 12 & 16 & 16 & 1996 & 68 & 66 & 74 & 62 & 68 \\
\hline 1997 & 13 & 23 & 21 & - & 19 & 1997 & 66 & 60 & 50 & - & 59 \\
\hline 1998 & 30 & 26 & 23 & 17 & 24 & 1998 & 63 & 59 & 71 & 59 & 63 \\
\hline 1999 & 39 & 29 & 14 & 31 & 28 & 1999 & 43 & 44 & 75 & 47 & 52 \\
\hline 2000 & 23 & 20 & 36 & 28 & 27 & 2000 & 45 & 50 & 38 & 39 & 43 \\
\hline 2001 & 28 & 29 & 25 & 27 & 27 & 1 & 44 & 33 & 41 & 45 & 41 \\
\hline 2002 & 30 & 17 & 32 & 26 & 26 & 2002 & - & 46 & 54 & 35 & 45 \\
\hline 2003 & 30 & 25 & 24 & 14 & 23 & 2003 & 39 & 47 & 53 & 45 & 46 \\
\hline 2004 & 33 & 25 & 39 & 37 & 33 & 2004 & 27 & 38 & 45 & 50 & 40 \\
\hline 2005 & 20 & 26 & 18 & 22 & 22 & 2005 & 52 & 56 & 52 & 52 & 53 \\
\hline 2006 & 24 & 26 & 28 & 26 & 26 & 2006 & 32 & 41 & 24 & 47 & 36 \\
\hline 2007 & - & 27 & 25 & 18 & 23 & 2007 & - & 41 & 37 & 42 & 40 \\
\hline 2008 & 26 & - & 29 & 28 & 28 & 8 & 48 & - & 47 & 28 & 41 \\
\hline 2009 & 16 & 15 & 16 & 10 & 14 & 2009 & 37 & 59 & 50 & 57 & 51 \\
\hline 2010 & 13 & 7 & 19 & 29 & 17 & 2010 & 39 & 67 & 38 & 38 & 46 \\
\hline 2011 & 20 & 19 & 22 & 21 & 20 & 2011 & 40 & 54 & 41 & 50 & 46 \\
\hline 2012 & 35 & 20 & 17 & 18 & 22 & 2012 & 40 & 40 & 52 & 41 & 43 \\
\hline 2013 & 28 & 17 & 30 & 26 & 25 & 2013 & 43 & 58 & 50 & 47 & 49 \\
\hline 2014 & 25 & 24 & 28 & 12 & 22 & 2014 & 38 & 24 & 22 & 47 & 33 \\
\hline 2015 & 10 & 34 & 14 & 38 & 24 & 2015 & 50 & 34 & 50 & 19 & 38 \\
\hline 2016 & 30 & 22 & 31 & 27 & 27 & 2016 & 20 & 50 & 32 & 20 & 31 \\
\hline 2017 & 24 & 19 & 21 & 13 & 19 & 2017 & 30 & 72 & 42 & 44 & 47 \\
\hline 2018 & 23 & 39 & 55 & & & 2018 & 31 & 46 & 36 & & \\
\hline
\end{tabular}




\begin{tabular}{|c|c|c|c|c|c|c|c|c|c|c|c|}
\hline Year & $\mathrm{IQ}$ & $\mathrm{IIQ}$ & $\mathrm{IIIQ}$ & IV Q & Annual & Year & $\mathrm{IQ}$ & $\mathrm{IIQ}$ & $\mathrm{IIIQ}$ & IV Q & Annual \\
\hline \multicolumn{6}{|c|}{$\begin{array}{l}\text { 162. Index of comprehension of government's } \\
\text { economic policy of last 3-4 months, agriculture (100/0 } \\
\text { - universal and complete comprehension/ non- } \\
\text { comprehension by enterprise directors } \\
\text { 162. Индекс понимания экономической политики } \\
\text { правительства последних } 3-4-\text {-х месяцев, сельское } \\
\text { хозяйство (100/0 - всеобщее и полное понимание } \\
\text { непонимание руководителями предприятий). }\end{array}$} & \multicolumn{6}{|c|}{$\begin{array}{l}\text { 163. Share of enterprise directors approving of } \\
\text { government's economic policy of last 3-4 months, } \\
\text { agriculture, \%) } \\
\text { 163. Доля руководителей, одобряющих } \\
\text { экономическую политику правительства за } \\
\text { последние } 3-4 \text { месяца, } \\
\text { сельское хозяйство (\%) }\end{array}$} \\
\hline 1993 & 39 & 41 & 31 & 44 & 39 & 1993 & 10 & 14 & 9 & 8 & 10 \\
\hline 1994 & 33 & 34 & 29 & 33 & 32 & 1994 & 5 & 7 & 8 & 0 & 5 \\
\hline 1995 & 27 & - & 26 & 35 & $29^{*}$ & 1995 & 0 & - & 2 & 4 & 2 \\
\hline 1996 & 30 & 31 & 26 & 33 & 30 & 1996 & 3 & 4 & 0 & 0 & 2 \\
\hline 1997 & 28 & 35 & 39 & - & $34^{*}$ & 1997 & 0 & 0 & 0 & - & 0 \\
\hline 1998 & 41 & 37 & 34 & 35 & 37 & 1998 & 0 & 3 & 3 & 0 & 1 \\
\hline 1999 & 49 & 41 & 25 & 40 & 39 & 1999 & 19 & 17 & 7 & 5 & 12 \\
\hline 2000 & 39 & 38 & 46 & 43 & 41 & 2000 & 9 & 13 & 5 & 6 & 8 \\
\hline 2001 & 44 & 47 & 44 & 43 & 44 & 2001 & 13 & 5 & 7 & 9 & 8 \\
\hline 2002 & - & 40 & 37 & 43 & 40 & 2002 & 19 & 7 & 7 & 0 & 8 \\
\hline 2003 & 44 & 42 & 41 & 37 & 41 & 2003 & 6 & 4 & 3 & 0 & 3 \\
\hline 2004 & 54 & 43 & 44 & 42 & 45 & 2004 & 3 & 6 & 3 & 7 & 5 \\
\hline 2005 & 38 & 33 & 36 & 38 & 36 & 2005 & 0 & 6 & 3 & 10 & 5 \\
\hline 2006 & 47 & 44 & 52 & 39 & 46 & 2006 & 13 & 4 & 4 & 11 & 8 \\
\hline 2007 & - & 47 & 46 & 40 & 44 & 2007 & - & 14 & 13 & 6 & 11 \\
\hline 2008 & 42 & - & 43 & 46 & 44 & 2008 & 21 & - & 14 & 14 & 16 \\
\hline 2009 & 42 & 35 & 35 & 32 & 36 & 2009 & . & 6 & 3 & 5 & 4 \\
\hline 2010 & 41 & 29 & 40 & 44 & 38 & 2010 & 8 & 8 & 4 & 10 & 7 \\
\hline 2011 & 41 & 38 & 41 & 36 & 39 & 2011 & 8 & 4 & 3 & 0 & 4 \\
\hline 2012 & 45 & 42 & 34 & 41 & 41 & 2012 & 10 & 4 & 0 & 6 & 5 \\
\hline 2013 & 45 & 33 & 43 & 39 & 40 & 2013 & 14 & 8 & 16 & 6 & 11 \\
\hline 2014 & 43 & 49 & 49 & 37 & 4 & 2014 & 25 & 0 & 11 & 6 & 11 \\
\hline 2015 & 35 & 49 & 35 & 43 & 41 & 2015 & 0 & 24 & 0 & 19 & 11 \\
\hline 2016 & 49 & 38 & 45 & 50 & 45 & 2016 & 15 & 17 & 6 & 20 & 14 \\
\hline 2017 & 46 & 30 & 39 & 36 & 38 & 2017 & 6 & 15 & 14 & 13 & 12 \\
\hline 2018 & 42 & 48 & 48 & & & 2018 & 8 & 23 & 25 & & \\
\hline
\end{tabular}




\begin{tabular}{|c|c|c|c|c|c|c|c|c|c|c|c|}
\hline Year & $\mathrm{IQ}$ & II Q & III Q & IVQ & \begin{tabular}{|l} 
Annual \\
\end{tabular} & Year & $I Q$ & $\| \mathrm{Q}$ & $\mathrm{IIIQ}$ & IVQ & \begin{tabular}{|l|l} 
Annual \\
\end{tabular} \\
\hline \multicolumn{6}{|c|}{$\begin{array}{c}\text { 164. Share of enterprise directors disapproving of } \\
\text { government's economic policy of last 3-4 months, } \\
\text { agriculture, (\%) } \\
\text { 164. Доля руководителей, не одобряющих } \\
\text { экономическую политику правительства за } \\
\text { последние 3-4 месяца, } \\
\text { сельское хозяйство (\%) }\end{array}$} & \multicolumn{6}{|c|}{$\begin{array}{l}\text { 165. Index of approving of government's economic policy } \\
\text { of last 3-4 months, agriculture (100/0 - universal and } \\
\text { complete approval/ disapproval by } \\
\text { enterprisedirectors) } \\
\text { 165. Индекс одобрения экономической политики } \\
\text { правительства последних 3-4-х месяцев, сельское } \\
\text { хозяйство (100 /0 - всеобщее и полное одобрение / } \\
\text { неоообрение руководителями предприятий). } \\
\end{array}$} \\
\hline $\begin{array}{l}1993 \\
1994 \\
1995 \\
1996 \\
1997 \\
1998 \\
1999 \\
2000 \\
2001 \\
2002 \\
2003 \\
2004 \\
2005 \\
2006 \\
2007 \\
2008 \\
2009 \\
2010 \\
2011 \\
2012 \\
2013 \\
2014 \\
2015 \\
2016 \\
2017 \\
2018\end{array}$ & $\begin{array}{l}46 \\
79 \\
80 \\
78 \\
80 \\
95 \\
50 \\
60 \\
44 \\
44 \\
66 \\
63 \\
72 \\
63 \\
- \\
42 \\
48 \\
26 \\
40 \\
55 \\
50 \\
50 \\
50 \\
35 \\
59 \\
54\end{array}$ & $\begin{array}{l}56 \\
74 \\
- \\
75 \\
84 \\
73 \\
52 \\
51 \\
43 \\
53 \\
65 \\
59 \\
75 \\
54 \\
63 \\
- \\
39 \\
54 \\
50 \\
34 \\
58 \\
58 \\
34 \\
55 \\
55 \\
39\end{array}$ & $\begin{array}{l}66 \\
71 \\
84 \\
93 \\
73 \\
80 \\
79 \\
51 \\
50 \\
72 \\
70 \\
68 \\
66 \\
56 \\
50 \\
48 \\
45 \\
56 \\
53 \\
55 \\
63 \\
39 \\
35 \\
38 \\
35 \\
50\end{array}$ & $\begin{array}{l}70 \\
77 \\
76 \\
84 \\
-8 \\
68 \\
65 \\
59 \\
51 \\
57 \\
64 \\
60 \\
63 \\
67 \\
48 \\
42 \\
62\end{array}$ & $\begin{array}{l}59 \\
75 \\
80 \\
82 \\
79 \\
79 \\
62 \\
55 \\
47 \\
56 \\
66 \\
63 \\
69 \\
60 \\
54 \\
44 \\
48 \\
47 \\
52 \\
49 \\
58 \\
49 \\
38 \\
39 \\
52\end{array}$ & $\begin{array}{l}1993 \\
1994 \\
1995 \\
1996 \\
1997 \\
1998 \\
1999 \\
2000 \\
2001 \\
2002 \\
2003 \\
2004 \\
2005 \\
2006 \\
2007 \\
2008 \\
2009 \\
2010 \\
2011 \\
2012 \\
2013 \\
2014 \\
2015 \\
2016 \\
2017 \\
2018\end{array}$ & $\begin{array}{l}35 \\
19 \\
20 \\
20 \\
17 \\
18 \\
37 \\
29 \\
36 \\
38 \\
24 \\
29 \\
17 \\
33 \\
- \\
40 \\
36 \\
45 \\
38 \\
34 \\
38 \\
39 \\
33 \\
41 \\
34 \\
33\end{array}$ & $\begin{array}{l}31 \\
21 \\
- \\
22 \\
19 \\
24 \\
34 \\
36 \\
35 \\
34 \\
26 \\
30 \\
20 \\
31 \\
31 \\
- \\
39 \\
33 \\
34 \\
40 \\
30 \\
34 \\
47 \\
35 \\
33 \\
38\end{array}$ & $\begin{array}{l}26 \\
28 \\
16 \\
13 \\
21 \\
18 \\
20 \\
33 \\
33 \\
24 \\
23 \\
23 \\
25 \\
32 \\
40 \\
37 \\
35 \\
31 \\
33 \\
33 \\
32 \\
40 \\
35 \\
39 \\
39 \\
36\end{array}$ & $\begin{array}{l}24 \\
22 \\
23 \\
18 \\
- \\
23 \\
28 \\
28 \\
32 \\
30 \\
26 \\
28 \\
27 \\
29 \\
34 \\
37 \\
29 \\
34 \\
27 \\
34 \\
28 \\
35 \\
54 \\
46 \\
30\end{array}$ & $\begin{array}{l}29 \\
22 \\
19 \\
18 \\
19 \\
21 \\
30 \\
31 \\
34 \\
32 \\
25 \\
27 \\
22 \\
31 \\
35 \\
38 \\
35 \\
36 \\
33 \\
35 \\
32 \\
37 \\
42 \\
40 \\
34\end{array}$ \\
\hline \multicolumn{6}{|c|}{$\begin{array}{l}\text { 166. Limitations to capital investments: shortage of } \\
\text { financial resources, current situation, agriculture } \\
\text { (share of enterprises, \%) } \\
\text { 166. Ограничения капитальных вложений: нехватка } \\
\text { финансовых ресурсов, текущая ситуация, сельское } \\
\text { хозяйство (доля предпиятий, \%) }\end{array}$} & \multicolumn{6}{|c|}{$\begin{array}{l}\text { 167. Limitations to capital investments: high prices of } \\
\text { equipment and construction, agriculture (share of } \\
\text { enterprises, \%) } \\
\text { 167. Ограничения капитальных вложений: } \\
\text { высокие цены на оборудование и строительство, } \\
\text { сельское хозяйство (доля предприятий, \%) }\end{array}$} \\
\hline $\begin{array}{l}1993 \\
1994 \\
1995 \\
1996 \\
1997 \\
1998 \\
1999 \\
2000 \\
2001 \\
2002 \\
2003 \\
2004 \\
2005 \\
2006 \\
2007 \\
2008 \\
2009 \\
2010 \\
2011 \\
2012 \\
2013 \\
2014 \\
2015 \\
2016\end{array}$ & $\begin{array}{l}71 \\
59 \\
62 \\
72 \\
91 \\
83 \\
87 \\
87 \\
88 \\
83 \\
85 \\
87 \\
92 \\
83 \\
77 \\
83 \\
79 \\
79 \\
83 \\
78 \\
80 \\
75 \\
88 \\
67 \\
72 \\
89\end{array}$ & $\begin{array}{l}78 \\
70 \\
80 \\
75 \\
80 \\
89 \\
91 \\
84 \\
78 \\
88 \\
90 \\
93 \\
96 \\
83 \\
94 \\
73 \\
65 \\
79 \\
79 \\
86 \\
79 \\
81 \\
79 \\
94 \\
81 \\
67\end{array}$ & $\begin{array}{l}78 \\
91 \\
89 \\
88 \\
87 \\
91 \\
95 \\
87 \\
94 \\
79 \\
86 \\
89 \\
82 \\
80 \\
95 \\
92 \\
87 \\
77 \\
77 \\
85 \\
83 \\
75 \\
92\end{array}$ & $\begin{array}{l}76 \\
87 \\
85 \\
88 \\
79 \\
79 \\
80 \\
85 \\
80 \\
64 \\
82 \\
73\end{array}$ & $\begin{array}{l}82 \\
87 \\
81 \\
78 \\
83 \\
83 \\
83 \\
80 \\
78 \\
79 \\
81 \\
75\end{array}$ & $\begin{array}{l}2012 \\
2013 \\
2014 \\
2015 \\
2016 \\
2017 \\
2018\end{array}$ & $\begin{array}{l}61 \\
67 \\
74 \\
77 \\
54 \\
71 \\
53 \\
54 \\
70 \\
60 \\
65 \\
65 \\
44 \\
56 \\
26\end{array}$ & $\begin{array}{l}71 \\
73 \\
74 \\
56 \\
71 \\
65 \\
44 \\
60\end{array}$ & $\begin{array}{l}79 \\
84 \\
78 \\
65 \\
73 \\
60 \\
82 \\
82 \\
82 \\
66 \\
80 \\
65 \\
71 \\
64 \\
61 \\
82 \\
84 \\
60 \\
67 \\
78 \\
68 \\
62 \\
77 \\
83 \\
58 \\
67\end{array}$ & $\begin{array}{l}63 \\
80 \\
60 \\
75 \\
64 \\
55 \\
68\end{array}$ & $\begin{array}{l}73 \\
75 \\
77 \\
70 \\
74 \\
67 \\
76 \\
71 \\
75 \\
70 \\
68 \\
68 \\
71 \\
69 \\
64 \\
65 \\
70 \\
58 \\
64 \\
75 \\
66 \\
65 \\
69 \\
62 \\
57\end{array}$ \\
\hline
\end{tabular}




\begin{tabular}{|c|c|c|c|c|c|c|c|c|c|c|c|}
\hline Year & $1 \mathrm{IQ}$ & $\| \mathrm{Q}$ & $\mathrm{IIIQ}$ & $I V Q$ & Annual & Year & $\mathrm{IQ}$ & $\| \mathrm{II}$ & III Q & $\overline{I V Q}$ & Annual \\
\hline \multicolumn{6}{|c|}{$\begin{array}{l}\text { 168. Limitations to capital investments: high bank } \\
\text { interest, current situation, agriculture (share of } \\
\text { enterprises, \%) } \\
\text { 168. Ограничения капитальных вложений: высокий } \\
\text { банковский процент, текущая ситуация, сельское } \\
\text { хозяйство (доля предприятий, \%) }\end{array}$} & \multicolumn{6}{|c|}{$\begin{array}{l}\text { 169. Limitations to capital investments: high } \\
\text { indebtedness, current situation, agriculture (share of } \\
\text { enterprises, \%) } \\
\text { 169. Ограничения капитальных вложений: } \\
\text { большая задолженность, текущая ситуация, } \\
\text { сельское хозяйство (доля предприятий, \%) }\end{array}$} \\
\hline 1993 & 41 & 44 & 54 & 53 & 48 & 1993 & 16 & 20 & 32 & 35 & 26 \\
\hline 1994 & 64 & 60 & 69 & 49 & 60 & 1994 & 29 & 20 & 18 & 25 & 23 \\
\hline 1995 & 55 & 43 & 45 & 58 & 50 & 1995 & 29 & 22 & 23 & 22 & 24 \\
\hline 1996 & 39 & 32 & 39 & 37 & 37 & 1996 & 39 & 27 & 37 & 42 & 36 \\
\hline 1997 & 28 & 30 & 30 & 29 & 29 & 1997 & 36 & 43 & 24 & 42 & 36 \\
\hline 1998 & 13 & 30 & 26 & 21 & 23 & 1998 & 33 & 32 & 40 & 26 & 33 \\
\hline 1999 & 31 & 20 & 24 & 27 & 23 & 1999 & 33 & 38 & 31 & 44 & 36 \\
\hline 2000 & 19 & 27 & 26 & 27 & 25 & 2000 & 37 & 24 & 32 & 30 & 31 \\
\hline 2001 & 31 & 36 & 25 & 36 & 32 & 2001 & 26 & 28 & 36 & 40 & 32 \\
\hline 2002 & 25 & 21 & 34 & 33 & 28 & 2002 & 22 & 35 & 32 & 42 & 33 \\
\hline 2003 & 33 & 25 & 13 & 30 & 25 & 2003 & 50 & 30 & 37 & 40 & 39 \\
\hline 2004 & 29 & 41 & 29 & 25 & 31 & 2004 & 35 & 31 & 35 & 28 & 32 \\
\hline 2005 & 28 & 33 & 25 & 43 & 32 & 2005 & 28 & 22 & 36 & 46 & 33 \\
\hline 2006 & 22 & 39 & 27 & 41 & 32 & 2006 & 35 & 33 & 27 & 24 & 30 \\
\hline 2007 & 18 & 39 & 50 & 35 & 35 & 2007 & 36 & 33 & 39 & 30 & 34 \\
\hline 2008 & 42 & 47 & 32 & 42 & 41 & 2008 & 33 & 27 & 18 & 19 & 24 \\
\hline 2009 & 21 & 17 & 32 & 8 & 20 & 2009 & 21 & 30 & 12 & 35 & 24 \\
\hline 2010 & 24 & 21 & 25 & 21 & 23 & 2010 & 35 & 16 & 25 & 29 & 26 \\
\hline 2011 & 33 & 21 & 21 & 29 & 26 & 2011 & 33 & 14 & 29 & 21 & 24 \\
\hline 2012 & 39 & 23 & 39 & 50 & 38 & 2012 & 17 & 23 & 22 & 5 & 17 \\
\hline 2013 & 40 & 26 & 41 & 35 & 36 & 2013 & 16 & 16 & 14 & 30 & 19 \\
\hline 2014 & 45 & 44 & 38 & 40 & 42 & 2014 & 15 & 13 & 8 & 15 & 13 \\
\hline 2015 & 24 & 57 & 69 & 29 & 45 & 2015 & 18 & 21 & 8 & 14 & 15 \\
\hline 2016 & 33 & 24 & 42 & 36 & 34 & 2016 & 11 & 18 & 17 & 9 & 14 \\
\hline 2017 & 24 & 25 & 42 & 32 & 31 & 2017 & 20 & 13 & 17 & 23 & 18 \\
\hline 2018 & 16 & 13 & 25 & & & 2018 & 5 & 20 & 17 & & \\
\hline \multicolumn{6}{|c|}{$\begin{array}{l}170 \text {. Limitations to capital investments: excessive } \\
\text { production capacities, current situation, agriculture } \\
\text { (share of enterprises, \%) } \\
\text { 170. Ограничения капитальных вложений: избыток } \\
\text { производственных мощностей, текущая ситуация, } \\
\text { сельское хозяйство (доля предприятий, \%) }\end{array}$} & \multicolumn{6}{|c|}{$\begin{array}{l}\text { 171. Limitations to capital investments: insufficient } \\
\text { profitability of investment projects, current situation, } \\
\text { agriculture (share of enterprises, \%) } \\
\text { 171. Ограничения капитальных вложений: } \\
\text { недостаточная прибыльность инвестиционных } \\
\text { проектов, текущая ситуация, сельское хозяйство } \\
\text { (доля предприятий, \%) }\end{array}$} \\
\hline 1993 & 2 & 4 & 2 & 6 & 4 & 1993 & - & - & 0 & 3 & - \\
\hline & 2 & 0 & 4 & 3 & 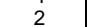 & 1994 & 5 & 3 & 2 & 8 & 5 \\
\hline & 5 & 4 & 10 & 0 & 5 & & 13 & 10 & 8 & 8 & 10 \\
\hline & 4 & 4 & 6 & 2 & 4 & & 11 & 18 & 12 & 2 & 11 \\
\hline & 2 & 0 & 0 & 6 & 2 & 7 & 4 & 7 & 9 & 6 & 7 \\
\hline & 7 & 3 & 6 & 4 & 5 & & 7 & 5 & 11 & 12 & 9 \\
\hline & 2 & 0 & 2 & 0 & 1 & & 6 & 7 & 10 & 4 & 7 \\
\hline & 2 & 2 & 5 & 3 & 3 & & 2 & 4 & 5 & 8 & 5 \\
\hline & 2 & 0 & 5 & 4 & 3 & & 2 & 8 & 7 & 12 & 7 \\
\hline & 3 & 0 & 3 & 4 & 3 & & 6 & 0 & 8 & 4 & 5 \\
\hline & 0 & 5 & 13 & 5 & 6 & & 7 & 15 & 7 & 10 & 10 \\
\hline & 3 & 0 & 3 & 8 & 4 & & 10 & 3 & 12 & 8 & 8 \\
\hline & 5 & 4 & 4 & $\begin{array}{l}0 \\
4\end{array}$ & 4 & & 13 & 7 & 4 & $\begin{array}{l}\circ \\
7\end{array}$ & $\begin{array}{l}\circ \\
8\end{array}$ \\
\hline & 9 & 0 & 0 & 0 & 2 & & 0 & 6 & 18 & 17 & 10 \\
\hline & 0 & 0 & 0 & 0 & 0 & & 23 & 6 & 6 & 9 & 11 \\
\hline & 0 & 0 & 0 & 0 & 0 & & 17 & 0 & 9 & 8 & 9 \\
\hline & 3 & 0 & 4 & 0 & 2 & & 12 & 4 & 8 & 8 & 8 \\
\hline & 6 & 5 & 0 & 13 & 6 & & 6 & 5 & 0 & 4 & 4 \\
\hline & 4 & 0 & 4 & 0 & 2 & & 4 & 14 & 8 & 8 & 8 \\
\hline & 4 & 0 & 0 & 0 & 1 & & 4 & 0 & 0 & 5 & 2 \\
\hline & 0 & 0 & 0 & 5 & 1 & & 8 & 0 & 0 & 10 & 4 \\
\hline 20 & 5 & 0 & 4 & 0 & 2 & & 15 & 6 & 4 & 15 & 10 \\
\hline & 0 & 0 & 8 & 0 & 2 & & 6 & $0^{*}$ & 0 & 29 & 9 \\
\hline 20 & 0 & 6 & 8 & 9 & 6 & & 11 & 18 & 0 & 0 & 7 \\
\hline 20 & 0 & 6 & 0 & 0 & 2 & 2017 & 12 & 13 & 17 & 14 & 14 \\
\hline 2018 & 0 & 7 & 8 & & & 2018 & 11 & 7 & 0 & & \\
\hline
\end{tabular}

